\title{
Nodal Statistics of Planar Random Waves
}

\author{
Ivan Nourdin $^{1}\left(\mathbb{D}\right.$, Giovanni Peccati $^{1}$, Maurizia Rossi $^{2}$ \\ 1 Unité de Recherche en Mathématiques, Université du Luxembourg, Esch-sur-Alzette, Luxembourg. \\ E-mail: ivan.nourdin@uni.lu \\ 2 Dipartimento di Matematica, Università di Pisa, Pisa, Italy
}

Received: 7 September 2017 / Accepted: 25 February 2019

Published online: 16 May 2019 - @ S Springer-Verlag GmbH Germany, part of Springer Nature 2019

\begin{abstract}
We consider Berry's random planar wave model (1977) for a positive Laplace eigenvalue $E>0$, both in the real and complex case, and prove limit theorems for the nodal statistics associated with a smooth compact domain, in the high-energy limit $(E \rightarrow \infty)$. Our main result is that both the nodal length (real case) and the number of nodal intersections (complex case) verify a Central Limit Theorem, which is in sharp contrast with the non-Gaussian behaviour observed for real and complex arithmetic random waves on the flat 2-torus, see Marinucci et al. (2016) and Dalmao et al. (2016). Our findings can be naturally reformulated in terms of the nodal statistics of a single random wave restricted to a compact domain diverging to the whole plane. As such, they can be fruitfully combined with the recent results by Canzani and Hanin (2016), in order to show that, at any point of isotropic scaling and for energy levels diverging sufficently fast, the nodal length of any Gaussian pullback monochromatic wave verifies a central limit theorem with the same scaling as Berry's model. As a remarkable byproduct of our analysis, we rigorously confirm the asymptotic behaviour for the variances of the nodal length and of the number of nodal intersections of isotropic random waves, as derived in Berry (2002).
\end{abstract}

\section{Introduction}

The aim of the present paper is to prove second order asymptotic results, in the highenergy limit, for the nodal statistics associated with the restriction of the (real and complex) Berry's random wave model [Ber02] to a smooth compact domain of $\mathbb{R}^{2}$. Our main result is a Central Limit Theorem (CLT) for both quantities (see Theorems 1.1 and 1.4), yielding as a by-product a rigorous and self-contained explanation of the cancellation phenomena for the variance asymptotics of nodal lengths and nodal intersections first detected in [Ber02]; this complements in particular the main findings of [Wig10].

As explained below, our techniques will show that the cancellation phenomena detected in [Ber02] can be explained by the partial cancellation of lower order Wiener- 
Itô chaotic projections. In particular, our findings represent a substantial addition to a rapidly growing line of research, focussing on the analysis of nodal quantities by means of Wiener-Itô chaotic expansions and associated techniques-see e.g. [CMW16a, CMW16b,CM16,DNPR16,MPRW16,MRW17,MW11,PR17,RoW17]. The central limit results proved in this paper are in sharp contrast with the non-central and nonuniversal limit theorems established in [DNPR16,MPRW16] for arithmetic random waves on the flat 2-torus, and mirror the CLTs for random spherical harmonics established in [MRW17]. To the best of our knowledge, our findings represent the first high-energy central limit theorems for nodal quantities associated with random Laplace eigenfunctions defined on the subset of a non-compact manifold.

As discussed in Sect. 1.4, our results can be naturally reformulated in terms of the nodal length and the nodal intersections of a single random wave, restricted to a compact window increasing to the whole plane. As such, they can be fruitfully combined with the findings of [CH16a], in order to prove CLTs for the nodal length of generic pullback random waves, locally determined by Riemaniann monochromatic waves (on a general compact manifold) at a given point of isotropic scaling - see Theorem 1.8 below.

Further motivations and connections with the existing literature will be discussed in the sections to follow.

Some conventions. For the rest of the paper, we assume that all random objects are defined on a common probability space $(\Omega, \mathcal{F}, \mathbb{P})$, with $\mathbb{E}$ denoting expectation with respect to $\mathbb{P}$. We use the symbol $\stackrel{d}{\longrightarrow}$ to denote convergence in distribution, and the symbol $\stackrel{\text { a.s. }}{\longrightarrow}$ to denote $\mathbb{P}$-almost sure convergence. Given two positive sequences $\left\{a_{n}\right\}$, $\left\{b_{n}\right\}$, we write $a_{n} \sim b_{n}$ if $a_{n} / b_{n} \rightarrow 1$, as $n \rightarrow \infty$.

1.1. Berry's random wave model. In [Ber77], Berry argued that, at least for classically chaotic quantum billiards, wavefunctions in the high-energy limit locally look like random superpositions of independent plane waves, having all the same wavenumber, say $k$, but different directions. According to [Ber02, formula (6)], such a superposition has the form

$$
u_{J ; k}(x):=\sqrt{\frac{2}{J}} \sum_{j=1}^{J} \cos \left(k x_{1} \cos \theta_{j}+k x_{2} \sin \theta_{j}+\phi_{j}\right), \quad J \gg 1,
$$

where $x=\left(x_{1}, x_{2}\right) \in \mathbb{R}^{2}$, and $\theta_{j}$ and $\phi_{j}$ are, respectively, random directions and random phases such that $\left(\theta_{1}, \phi_{1}, \ldots, \theta_{J}, \phi_{J}\right)$ are i.i.d. uniform random variables on $\left.[0,2 \pi]\right)$. For dynamical systems with time-reversal symmetry, these plane waves are real, while in the absence of time-reversal symmetry, for instance when the billiard is open, they are complex functions:

$$
u_{J ; k}^{\mathbb{C}}(x):=u_{J ; k}(x)+i v_{J ; k}(x),
$$

where $v_{J ; k}(x)$ is given by formula (1.1) with the cosine replaced by the sine, and the random vector $\left(\theta_{1}, \phi_{1}, \ldots, \theta_{J}, \phi_{J}\right)$ is defined as above; see again [Ber02], as well as the surveys [DOP09,UR13] and the references therein.

The sequence $\left\{u_{J ; k}\right\}_{J}$ in (1.1) converges in the sense of finite-dimensional distributions, as $J \rightarrow+\infty$, to the centered isotropic Gaussian field $b_{k}=\left\{b_{k}(x): x \in \mathbb{R}^{2}\right\}$, with covariance kernel given by

$$
c^{k}(x, y)=c^{k}(x-y):=\mathbb{E}\left[b_{k}(x) b_{k}(y)\right]=J_{0}(k\|x-y\|), \quad x, y \in \mathbb{R}^{2},
$$


where $J_{0}$ denotes the zero-order Bessel function of the first kind:

$$
J_{0}(t)=\sum_{m=0}^{+\infty} \frac{(-1)^{m}}{(m !)^{2}}\left(\frac{t}{2}\right)^{2 m}, \quad t \in \mathbb{R} .
$$

Recall that $J_{0}$ is the only radial solution of the equation

$$
\Delta f+f=0
$$

such that $f(0)=1$, where $\Delta:=\partial^{2} / \partial x_{1}^{2}+\partial^{2} / \partial x_{2}^{2}$ denotes the Laplacian on the Euclidean plane.

It is a standard fact (see e.g. [AT, Theorem 5.7.3]) that we can represent $b_{k}$ as a random series

$$
b_{k}(x)=b_{k}(r, \theta)=\Re\left(\sum_{m=-\infty}^{+\infty} a_{m} J_{|m|}(k r) \mathrm{e}^{i m \theta}\right),
$$

using polar coordinates $(r, \theta)=x$, where $\Re$ denotes the real part, $a_{m}$ are i.i.d. complex Gaussian random variables such that $\mathbb{E}\left[a_{m}\right]=0$ and $\mathbb{E}\left[\left|a_{m}\right|^{2}\right]=2$, and $J_{\alpha}$ stands for the Bessel function of the first kind of order $\alpha$. The series (1.5) is a.s. convergent, and uniformly convergent on any compact set, and the sum is a real analytic function (this is due to the fact that the mapping $\alpha \mapsto J_{\alpha}(z)$ is asymptotically equivalent to $\alpha^{-1 / 2}(2 z / \pi \alpha)^{\alpha}$, as $\alpha \rightarrow+\infty$-see e.g. [AS64, formula (9.3.1)]). From (1.5) it follows also that $b_{k}$ is a.s. an eigenfunction of the Laplacian $\Delta$ on $\mathbb{R}^{2}$ with eigenvalue $-k^{2}$, i.e. $b_{k}$ solves the Helmholtz equation

$$
\Delta b_{k}(x)+k^{2} b_{k}(x)=0, \quad x \in \mathbb{R}^{2}
$$

A standard application e.g. of [AT, Theorem 5.7.2] also shows the following reverse statement: if $Y$ is an isotropic centered Gaussian field on the plane, with unit variance and such that $\Delta Y+k^{2} Y=0$, then necessarily $Y$ has the same distribution as $b_{k}$. This also shows that, for every $k>0$, the two Gaussian random functions $x \mapsto b_{k}(x)$ and $x \mapsto b_{1}(k x)$ have the same distribution.

The 'universal' random field $b_{k}$ is known as Berry's Random Wave Model, and is the main object of our paper. The complex version of $b_{k}$ we consider is the random field

$$
b_{k}^{\mathbb{C}}(x):=b_{k}(x)+i \widehat{b}_{k}(x), \quad x \in \mathbb{R}^{2},
$$

where $\widehat{b}_{k}$ is an independent copy of $b_{k}$. We observe that $b_{k}^{\mathbb{C}}$ can be represented as a random series as well, and that such a representation is obtained by removing the symbol $\mathfrak{R}$ on the right-hand side of (1.5). It follows in particular that $b_{k}^{\mathbb{C}}$ a.s. verifies the equation $\Delta b_{k}^{\mathbb{C}}+k^{2} b_{k}^{\mathbb{C}}=0$, that is, $b_{k}^{\mathbb{C}}$ is a.s. a complex-valued solution of the Helmholtz equation associated with the eigenvalue $-k^{2}$. 
1.2. Mean and variance of nodal statistics (Berry 2002). The principal focus of our analysis are the two nodal sets:

$$
b_{k}^{-1}(0):=\left\{x \in \mathbb{R}^{2}: b_{k}(x)=0\right\}, \text { and }\left(b_{k}^{\mathbb{C}}\right)^{-1}(0)=b_{k}^{-1}(0) \cap\left(\widehat{b}_{k}\right)^{-1}(0) .
$$

It is proved in Lemma 8.4 of Appendix A that $b_{k}^{-1}(0)$ is a.s. a union of smooth curves (called nodal lines), while $\left(b_{k}^{\mathbb{C}}\right)^{-1}(0)$ is a.s. composed of isolated points (often referred to as phase singularities or optical vortices - see [DOP09,UR13]).

In [Ber02], the distributions of the length $\mathbf{l}_{k}$ of the nodal lines of $b_{k}$ and of the number $\mathbf{n}_{k}$ of nodal points of its complex version, when restricted to some fixed billiard $\mathcal{D}$, were studied. In particular, for the means of the latter quantities, Berry found that

$$
\mathbb{E}\left[\mathbf{l}_{k}\right]=\frac{A k}{2 \sqrt{2}} \text { and } \mathbb{E}\left[\mathbf{n}_{k}\right]=\frac{A k^{2}}{4 \pi},
$$

where $A$ denotes the area of $\mathcal{D}$, while for their high-energy fluctuations, some semirigorous computations led to the following asymptotic relations, valid as $k \rightarrow \infty$ :

$$
\operatorname{Var}\left(\mathbf{l}_{k}\right) \sim \frac{A}{256 \pi} \log (k \sqrt{A}), \text { and } \operatorname{Var}\left(\mathbf{n}_{k}\right) \sim \frac{11 A k^{2}}{64 \pi^{3}} \log (k \sqrt{A}) .
$$

According to [Ber02], the unexpected logarithmic order of both variances in (1.8) is due to an "obscure cancellation phenomenon", corresponding to an exact simplification of several terms appearing in the Kac-Rice formula-see the discussion below-as applied to the computation of variances. As anticipated, our aim in this paper is to prove a CLT both for $\mathbf{l}_{k}$ and $\mathbf{n}_{k}$, yielding as a by-product a rigorous explanation of (1.8) in terms of the partial cancellation of lower order Wiener-Itô chaotic components.

1.3. Main results. In order to make more transparent the connection with some relevant parts of the recent literature (see Sect. 1.5), for the rest of the paper we set, for $E>0$,

$$
B_{E}(x):=b_{k}(x), \quad x \in \mathbb{R}^{2},
$$

where $k:=2 \pi \sqrt{E}$, in such a way that the covariance of $B_{E}$ is given by

$$
r^{E}(x, y)=r^{E}(x-y):=J_{0}(2 \pi \sqrt{E}\|x-y\|), x, y \in \mathbb{R}^{2} ;
$$

see (1.3). Analogously, for $E>0$ we write

$$
B_{E}^{\mathbb{C}}(x):=b_{k}^{\mathbb{C}}(x)=B_{E}(x)+i \widehat{B}_{E}(x), \quad x \in \mathbb{R}^{2},
$$

where $k=2 \pi \sqrt{E}$, and $\widehat{B}_{E}$ is an independent copy of $B_{E}$.

Let us now fix a $C^{1}$-convex body $\mathcal{D} \subset \mathbb{R}^{2}$ (that is: $\mathcal{D}$ is a compact convex set with $C^{1}$ boundary) such that $0 \in \mathcal{D}$ (i.e. the origin belongs to the interior of $\mathcal{D}$ ). The restriction of the zero set of $B_{E}$ to $\mathcal{D}$ is

$$
B_{E}^{-1}(0) \cap \mathcal{D}=\left\{x \in \mathcal{D}: B_{E}(x)=0\right\} .
$$

According to Lemma 8.4 in Appendix A, the set $B_{E}^{-1}(0)$ intersects the boundary $\partial \mathcal{D}$ in an a.s. finite number of points. The nodal length of $B_{E}$ restricted to $\mathcal{D}$ is the random variable

$$
\mathcal{L}_{E}:=\operatorname{length}\left(B_{E}^{-1}(0) \cap \mathcal{D}\right),
$$

which is square-integrable, by Lemma 3.3 below. The first main result of the present paper concerns the distribution of $\mathcal{L}_{E}$ in the high-energy limit. 
Theorem 1.1. The expectation of the nodal length $\mathcal{L}_{E}$ is

$$
\mathbb{E}\left[\mathcal{L}_{E}\right]=\operatorname{area}(\mathcal{D}) \frac{\pi}{\sqrt{2}} \sqrt{E},
$$

whereas the variance of $\mathcal{L}_{E}$ verifies the asymptotic relation

$$
\operatorname{Var}\left(\mathcal{L}_{E}\right) \sim \operatorname{area}(\mathcal{D}) \frac{1}{512 \pi} \log E, \quad E \rightarrow \infty .
$$

Moreover, as $E \rightarrow \infty$,

$$
\frac{\mathcal{L}_{E}-\mathbb{E}\left[\mathcal{L}_{E}\right]}{\sqrt{\operatorname{Var}\left(\mathcal{L}_{E}\right)}} \stackrel{d}{\rightarrow} Z,
$$

where $Z \sim \mathscr{N}(0,1)$ is a standard Gaussian random variable.

Remark 1.2. Relation (1.11) coincides with [Ber02, formula (19)] (and (1.7) above), whereas (1.12) is consistent with [Ber02, formula (28)] (and (1.8) above).

Remark 1.3. In what follows, we will use the relation

$$
\mathcal{L}_{E} \stackrel{d}{=} \frac{1}{2 \pi \sqrt{E}} \operatorname{length}\left(b_{1}^{-1}(0) \cap 2 \pi \sqrt{E} \cdot \mathcal{D}\right),
$$

where $\stackrel{d}{=}$ indicates equality in distribution and, for $a>0$, we set $a \cdot \mathcal{D}:=\left\{y \in \mathbb{R}^{2}\right.$ : $y=a x, x \in \mathcal{D}\}$. Such an equality in distribution is an immediate consequence of the integral representation of nodal lengths appearing e.g. in (2.23) below, as well as of the fact that, as random functions, $b_{1}(2 \pi \sqrt{E} x)$ and $B_{E}(x)$ have the same distribution for every $E>0$.

We now focus on the complex Berry's RWM $B_{E}^{\mathbb{C}}$, and study the nodal points (phase singularities) of $B_{E}^{\mathbb{C}}$ that belong to a $C^{1}$ convex body $\mathcal{D}$ such as the one considered above (in particular, the origin lies in the interior of $\mathcal{D}$ ). As already observed, one has that

$$
\left(B_{E}^{\mathbb{C}}\right)^{-1}(0)=B_{E}^{-1}(0) \cap \widehat{B}_{E}^{-1}(0),
$$

and the set $\left(B_{E}^{\mathbb{C}}\right)^{-1}(0) \cap \mathcal{D}$ consists $\mathbb{P}$-a.s. of a finite collection of points such that none of them belongs to the boundary $\partial \mathcal{D}$ (see Lemma 8.4). We are interested in the distribution of

$$
\mathcal{N}_{E}:=\#\left(\left(B_{E}^{\mathbb{C}}\right)^{-1}(0) \cap \mathcal{D}\right),
$$

for large values of $E$. Our second main result is the following:

Theorem 1.4. One has that

$$
\mathbb{E}\left[\mathcal{N}_{E}\right]=\operatorname{area}(\mathcal{D}) \pi E
$$

Moreover, as $E \rightarrow \infty$,

and

$$
\operatorname{Var}\left(\mathcal{N}_{E}\right) \sim \operatorname{area}(\mathcal{D}) \frac{11}{32 \pi} E \log E,
$$

$$
\frac{\mathcal{N}_{E}-\mathbb{E}\left[\mathcal{N}_{E}\right]}{\sqrt{\operatorname{Var}\left(\mathcal{N}_{E}\right)}} \stackrel{d}{\longrightarrow} Z,
$$

where $Z \sim \mathscr{N}(0,1)$ is a standard Gaussian random variable. 
Remark 1.5. Relation (1.15) coincides with [Ber02, (45)] (or (1.7)) above) whereas (1.16) is consistent with [Ber02, (50)] (or (1.8) above).

We will now show how Theorem 1.1 can be combined with the findings of [CH16a], in order to deduce local CLTs for pullback (monochromatic) random waves associated with a general Riemaniann manifold.

\subsection{Application to monochromatic random waves.}

1.4.1. Random waves on manifolds. Let $(\mathcal{M}, g)$ be a compact, smooth, Riemannian manifold of dimension 2. We write $\Delta_{g}$ to indicate the associated Laplace-Beltrami operator, and denote by $\left\{f_{j}: j \in \mathbb{N}\right\}$ an orthonormal basis of $L^{2}(\mathcal{M})$ composed of real-valued eigenfunctions of $\Delta_{g}$

$$
\Delta_{g} f_{j}+\lambda_{j}^{2} f_{j}=0
$$

where the corresponding eigenvalues are such that $0=\lambda_{0}<\lambda_{1} \leq \lambda_{2} \leq \ldots \uparrow \infty$. According to [CH16a,Zel09], the (Riemannian) monochromatic random wave on $M$ of parameter $\lambda$ is defined as the Gaussian random field

$$
\phi_{\lambda}(x):=\frac{1}{\sqrt{\operatorname{dim}\left(H_{c, \lambda}\right)}} \sum_{\lambda_{j} \in[\lambda, \lambda+c]} a_{j} f_{j}(x), \quad x \in M
$$

where $c \geq 0$ is a fixed parameter and the $a_{j}$ are i.i.d. standard Gaussian random variables, and

$$
H_{c, \lambda}:=\bigoplus_{\lambda_{j} \in[\lambda, \lambda+c]} \operatorname{Ker}\left(\Delta_{g}+\lambda_{j}^{2} \mathrm{Id}\right)
$$

where Id is the identity operator. The field $\phi_{\lambda}$ is centered Gaussian, and its covariance kernel is given by

$$
\begin{aligned}
K_{c, \lambda}(x, y) & :=\operatorname{Cov}\left(\phi_{\lambda}(x), \phi_{\lambda}(y)\right) \\
& =\frac{1}{\operatorname{dim}\left(H_{c, \lambda}\right)} \sum_{\lambda_{j} \in[\lambda, \lambda+c]} f_{j}(x) f_{j}(y), \quad x, y \in \mathcal{M} .
\end{aligned}
$$

"Short window" monochromatic random waves such as $\phi_{\lambda}$ (in the case $c=1$ and for manifolds of arbitrary dimension) were first introduced by Zelditch in [Zel09] as general approximate models of random Gaussian Laplace eigenfunctions defined on manifolds not necessarily having spectral multiplicities; see [CH16a] for further discussions. The case $c=0$ typically corresponds to manifolds with spectral multiplicities like the flat torus $\mathbb{R}^{2} / \mathbb{Z}^{2}$ or the round sphere $\mathbb{S}^{2}$, where one can consider models of random waves living on a single eigenspace (like arithmetic random waves [RW08], and random spherical harmonics [Wig10])—-see also the forthcoming Sect. 1.5. Plainly, for a generic metric on a smooth compact manifold $\mathcal{M}$, the eigenvalues $\lambda_{j}^{2}$ are simple, and one has to average on intervals $[\lambda, \lambda+c]$ such that $c>0$ in order to obtain a non-trivial probabilistic model. 
1.4.2. Pulback random waves and isotropic scaling We keep the notation introduced in the previous section, and follow closely [CH16a]. Fix $x \in \mathcal{M}$, and consider the tangent plane $T_{x} \mathcal{M}$ to the manifold at $x$. We define the pullback Riemannian random wave associated with $\phi_{\lambda}$ as the Gaussian random field on $T_{x} \mathcal{M}$ given by

$$
\phi_{\lambda}^{x}(u):=\phi_{\lambda}\left(\exp _{x}\left(\frac{u}{\lambda}\right)\right), \quad u \in T_{x} \mathcal{M}
$$

where $\exp _{x}: T_{x} \mathcal{M} \rightarrow \mathcal{M}$ is the exponential map at $x$. The planar field $\phi_{\lambda}^{x}$ is trivially centered and Gaussian and, using (1.18), its covariance kernel is given by

$$
K_{c, \lambda}^{x}(u, v)=K_{c, \lambda}\left(\exp _{x}\left(\frac{u}{\lambda}\right), \exp _{x}\left(\frac{v}{\lambda}\right)\right), \quad u, v \in T_{x} \mathcal{M} .
$$

Definition 1.6 (See [CH16a]). We say that $x \in \mathcal{M}$ is a point of isotropic scaling if, for every positive function $\lambda \mapsto r(\lambda)$ such that $r(\lambda)=o(\lambda)$, as $\lambda \rightarrow \infty$, one has that

$$
\sup _{u, v \in \mathbb{B}(r(\lambda))}\left|\partial^{\alpha} \partial^{\beta}\left[K_{c, \lambda}^{x}(u, v)-(2 \pi) J_{0}\left(\|u-v\|_{g_{x}}\right)\right]\right| \rightarrow 0, \quad \lambda \rightarrow \infty,
$$

where $\alpha, \beta \in \mathbb{N}^{2}$ are multi-indices labeling partial derivatives with respect to $u$ and $v$, respectively, $\|\cdot\|_{g_{x}}$ is the norm on $T_{x} \mathcal{M}$ induced by $g$, and $\mathbb{B}(r(\lambda))$ is the corresponding ball of radius $r(\lambda)$ containing the origin.

Sufficient conditions for a point $x$ to be of isotropic scaling are discussed e.g. in [CH16a, Sect. 2.5] or [CH16b]. In the case $c=0$, one can directly verify that every point $x \in \mathbb{S}^{2}$ is of isotropic scaling for the model of random spherical harmonics evoked above (see [Wig10]). Note that one can always choose coordinates around $x$ to have $g_{x}=\mathrm{Id}$, so that the limiting kernel in (1.19) coincides with $(2 \pi) \times c^{1}$ in (1.3). This implies in particular that, if $x$ is a point of isotropic scaling, then, as $\lambda \rightarrow \infty$, the planar field $\phi_{\lambda}^{x}$ converges to a multiple of Berry's model, namely $\sqrt{2 \pi} \cdot b_{1}$, in the sense of finite-dimensional distributions.

1.4.3. A second order result Keeping the same notation and assumptions as above, we now state a special case of [CH16a, Theorem 1], that we reformulate in a way that is adapted to the notation adopted in the present paper. To this end, for every $x \in \mathcal{M}$ we define

$$
\mathcal{Z}_{\lambda, E}^{x}:=\text { length }\left\{\left(\phi_{\lambda}^{x}\right)^{-1}(0) \cap \mathbb{B}(2 \pi \sqrt{E})\right\}, \quad E>0 .
$$

The next statement shows that, if $x$ is of isotropic scaling, then $\mathcal{Z}_{\lambda, E}^{x}$ behaves, for large values of $\lambda$ as the universal random quantity given by the nodal length of Berry's model $b_{1}$ restricted to the ball $\mathbb{B}(2 \pi \sqrt{E})$.

Theorem 1.7 (Special case of Theorem 1 in [CH16a]). Let $x$ be a point of isotropic scaling, and assume that coordinates have been chosen around $x$ in such a way that $g_{x}=$ Id. Fix $E>0$. Then, as $\lambda \rightarrow \infty$, the random variable $\mathcal{Z}_{\lambda, E}^{x}$ converges in distribution to

$$
\text { length }\left(b_{1}^{-1}(0) \cap \mathbb{B}(2 \pi \sqrt{E})\right) \quad\left(\stackrel{d}{=} \mathcal{L}_{E} \cdot 2 \pi \sqrt{E}\right),
$$

where the identity in distribution expressed between brackets follows from (1.13). 
The next statement is a direct consequence of Theorem 1.1, and provides a secondorder counterpart to Theorem 1.7, showing in particular that nodal lengths of pullback random waves inherit high-energy Gaussian fluctuations from Berry's model at any point of isotropic scaling. In order to make the statement more readable, we introduce the notation

$$
\widetilde{\mathcal{Z}}_{\lambda, E}^{x}:=\frac{\mathcal{Z}_{\lambda, E}^{x}}{2 \pi \sqrt{E}}
$$

Theorem 1.8 (CLT for the nodal length of pullback waves). Let $x$ be a point of isotropic scaling, and assume that coordinates have been chosen around $x$ in such a way that $g_{x}=$ Id. Let $\left\{E_{m}: m \geq 1\right\}$ be a sequence of positive numbers such that $E_{m} \rightarrow \infty$. Then, there exists a sequence $\left\{\lambda_{m}: m \geq 1\right\}$ such that

$$
\frac{\widetilde{\mathcal{Z}}_{\lambda_{m}, E_{m}}^{x}-\pi^{2} \sqrt{E_{m} / 2}}{\sqrt{\log \left(E_{m}\right) / 512}} \stackrel{d}{\longrightarrow} Z \sim \mathscr{N}(0,1) .
$$

Proof. Let $\mathbf{d}(\cdot, \cdot)$ be any distance metrizing the convergence in distribution between random variables (see e.g. [NP12, Appendix C]), and let $\epsilon(m), m \geq 1$, be a sequence of positive numbers such that $\epsilon(m) \rightarrow 0$. According to Theorem 1.7 , for every fixed $m$ there exists $\lambda_{m}>0$ such that

$$
\mathbf{d}\left(\frac{\widetilde{\mathcal{Z}}_{\lambda_{m}, E_{m}}^{x}-\pi^{2} \sqrt{E_{m} / 2}}{\sqrt{\log \left(E_{m}\right) / 512}}, \frac{\mathcal{L}_{E_{m}}-\pi^{2} \sqrt{E_{m} / 2}}{\sqrt{\log \left(E_{m}\right) / 512}}\right) \leq \epsilon(m) .
$$

From this relation we deduce that, for every $m$,

$$
\mathbf{d}\left(\frac{\widetilde{\mathcal{Z}}_{\lambda_{m}, E_{m}}-\pi^{2} \sqrt{E_{m} / 2}}{\sqrt{\log \left(E_{m}\right) / 512}}, Z\right) \leq \epsilon(m)+\mathbf{d}\left(\frac{\mathcal{L}_{E_{m}}-\pi^{2} \sqrt{E_{m} / 2}}{\sqrt{\log \left(E_{m}\right) / 512}}, Z\right),
$$

and the conclusion follows at once from Theorem 1.1.

It would be of course desirable to have some quantitative information about the sequence $\lambda_{m}, m \geq 1$ appearing in the previous statement, in particular connecting the asymptotic behaviour of $\lambda_{m}$ with the speed of divergence of $E_{m}$. Some preliminary computations have indicated us that (not suprisingly) in order to do so, one should have explicit upper bounds on the limiting relation (1.19), that one should exploit in order to deduce a quantitative version of Theorem 1.7. We prefer to think of this issue as a separate problem, and leave it open for further research.

1.5. Further related work. The distribution of the nodal length on the standard flat torus $\mathbb{T}^{2}$ and on the unit round sphere $\mathbb{S}^{2}$ was investigated in [RW08, KKW13, MPRW16, PR17] and [Bera85, Wig10,MRW17], respectively. Moreover, the distribution of the number of nodal points on $\mathbb{T}^{2}$ was studied in [DNPR16]. The first result in higher dimensional setting concerns the fine asymptotic behavior of the nodal area for 3dimensional "arithmetic random waves", i.e. Gaussian Laplace eigenfunctions on the 3-torus $\mathbb{T}^{3}:=\mathbb{R}^{3} / \mathbb{Z}^{3}$ (see [Cam17]).

Remember that, as mentioned in Sect. 1.4, since these manifolds have spectral degeneracies, one typically selects the value $c=0$ in (1.17) for defining a canonical model 
of Gaussian random waves. We will now describe in more detail the theoretical contributions contained in the references evoked above. A more technical comparison with the approach adopted in the present work is deferred to Sect. 2.2.

Nodal length of real arithmetic random waves. The eigenvalues of the Laplace operator on $\mathbb{T}^{2}$ are of the form $-4 \pi^{2} n$, where $n$ is an integer that can be represented as the sum of two integer squares. Write $S$ for the collection of all integers having this property, and, for $n \in S$, denote by $\Lambda_{n}$ the set of frequencies

$$
\Lambda_{n}=\left\{\xi \in \mathbb{Z}^{2}:\|\xi\|=\sqrt{n}\right\}
$$

and by $\mathcal{N}_{n}$ the cardinality of $\Lambda_{n}$ (that is, $\mathcal{N}_{n}$ is the multiplicity of $-4 \pi^{2} n$ ). For $n \in S$, consider the probability measure $\mu_{n}$ induced by $\Lambda_{n}$ on the unit circle $\mathbb{S}^{1}$ :

$$
\mu_{n}=\frac{1}{\mathcal{N}_{n}} \sum_{\xi \in \Lambda_{n}} \delta_{\xi / \sqrt{n}}
$$

Following [RW08], for $n \in S$, the toral random eigenfunction $T_{n}$ (or arithmetic random wave of order $n$ ) is defined as the centered Gaussian field on the torus whose covariance function is as follows: for $x, y \in \mathbb{T}^{2}$,

$$
\begin{aligned}
\operatorname{Cov}\left(T_{n}(x), T_{n}(y)\right) & =\frac{1}{\mathcal{N}_{n}} \sum_{\xi \in \Lambda_{n}} \mathrm{e}^{i 2 \pi\langle\xi, x-y\rangle} \\
& =\int_{\mathbb{S}^{1}} \mathrm{e}^{i 2 \pi \sqrt{n}\langle\theta, x-y\rangle} d \mu_{n}(\theta) .
\end{aligned}
$$

As discussed in [KKW13], there exists a density-1 subsequence $\left\{n_{j}: j \geq 1\right\} \in S$ such that, as $j \rightarrow+\infty$,

$$
\mu_{n_{j}} \Rightarrow d \theta / 2 \pi,
$$

where $d \theta$ denotes the uniform measure on the unit circle. Let us now set $\mathcal{L}_{n}:=$ length $\left(T_{n}^{-1}(0)\right)$. The expected nodal length was computed by Rudnick and Wigman [RW08]:

$$
\mathbb{E}\left[\mathcal{L}_{n}\right]=\frac{1}{2 \sqrt{2}} \sqrt{4 \pi^{2} n},
$$

while in [KKW13] the asymptotic variance, as $\mathcal{N}_{n} \rightarrow+\infty$, was proved to be

$$
\operatorname{Var}\left(\mathcal{L}_{n}\right) \sim \frac{1+\widehat{\mu_{n}}(4)^{2}}{512} \frac{4 \pi^{2} n}{\mathcal{N}_{n}^{2}},
$$

where $\widehat{\mu_{n}}(4)$ denotes the fourth Fourier coefficients of $\mu_{n}$. In order to have an asymptotic law for the variance, one should select a subsequence $\left\{n_{j}\right\}$ of energy levels such that (i) $\mathcal{N}_{n_{j}} \rightarrow+\infty$ and (ii) $\left|\widehat{\mu_{n}}(4)\right| \rightarrow \eta$, for some $\eta \in[0,1]$. Note that for each $\eta \in[0,1]$, there exists a subsequence $\left\{n_{j}\right\}$ such that both (i) and (ii) hold (see [KKW13,KW16]). For these subsequences, the asymptotic distribution of the nodal length was shown to be non-Gaussian in [MPRW16]:

$$
\frac{\mathcal{L}_{n_{j}}-\mathbb{E}\left[\mathcal{L}_{n_{j}}\right]}{\sqrt{\operatorname{Var}\left(\mathcal{L}_{n_{j}}\right)}} \stackrel{d}{\rightarrow} \frac{1}{2 \sqrt{1+\eta^{2}}}\left(2-(1-\eta) Z_{1}^{2}-(1+\eta) Z_{2}^{2}\right),
$$


where $Z_{1}$ and $Z_{2}$ are i.i.d. standard Gaussian random variables. A complete quantitative version (in Wasserstein distance) of (1.22) is given in [PR17]. Reference [RoW17] contains Limit Theorems for the intersection number of the nodal lines $T_{n}^{-1}(0)$ and a fixed deterministic curve with nowhere zero curvature.

Phase singularities of complex arithmetic random waves. For $n \in S$, let $\widehat{T}_{n}$ indicate an independent copy of the arithmetic random wave $T_{n}$ defined in the previous paragraph. In [DNPR16], the distribution of the cardinality $\mathcal{I}_{n}$ of the set of nodal intersections $T_{n}^{-1}(0) \cap \widehat{T}_{n}^{-1}(0)$ was investigated. One has that

$$
\mathbb{E}\left[\mathcal{I}_{n}\right]=\frac{4 \pi^{2} n}{4 \pi}=\pi n,
$$

while the asymptotic variance, as $\mathcal{N}_{n} \rightarrow+\infty$, is

$$
\operatorname{Var}\left(\mathcal{I}_{n}\right) \sim \frac{3 \widehat{\mu_{n}}(4)^{2}+5}{128 \pi^{2}} \frac{\left(4 \pi^{2} n\right)^{2}}{\mathcal{N}_{n}^{2}} .
$$

Also in this case the asymptotic distribution is non-Gaussian (and non-universal), indeed for $\left\{n_{j}\right\}$ such that $\mathcal{N}_{n_{j}} \rightarrow+\infty$ and $\left|\widehat{\mu_{n_{j}}}(4)\right| \rightarrow \eta \in[0,1]$, one has that

$$
\frac{\mathcal{I}_{n_{j}}-\mathbb{E}\left[\mathcal{I}_{n_{j}}\right]}{\sqrt{\operatorname{Var}\left(\mathcal{I}_{n_{j}}\right)}} \stackrel{d}{\rightarrow} \frac{1}{2 \sqrt{10+6 \eta^{2}}}\left(\frac{1+\eta}{2} A+\frac{1-\eta}{2} B-2(C-2)\right),
$$

where $A, B$ and $C$ are independent random variables such that $A \stackrel{d}{=} B \stackrel{d}{=} 2 Z_{1}^{2}+2 Z_{2}^{2}-4 Z_{3}^{2}$ while $C \stackrel{d}{=} Z_{1}^{2}+Z_{2}^{2}$ (where $Z_{1}, Z_{2}, Z_{3}$ are i.i.d. standard Gaussian random variables).

Nodal length of random spherical harmonics. The Laplacian eigenvalues on the twodimensional unit sphere are of the form $-\ell(\ell+1)$, where $\ell \in \mathbb{N}$, and the multiplicity of the $\ell$-th eigenvalue is $2 \ell+1$. The $\ell$-th random eigenfunction (random spherical harmonic) on $\mathbb{S}^{2}$ is a centered Gaussian field whose covariance kernel is

$$
\left.\operatorname{Cov}\left(T_{\ell}(x)\right), T_{\ell}(y)\right)=P_{\ell}(\cos d(x, y)), \quad x, y \in \mathbb{S}^{2},
$$

where $P_{\ell}$ denotes the $\ell$-th Legendre polynomial and $d(x, y)$ the geodesic distance between the two points $x$ and $y$ (see [MP11]). The mean of the nodal length $\mathcal{L}_{\ell}:=$ length $\left(T_{\ell}^{-1}(0)\right)$ was computed in [Bera85] as

$$
\mathbb{E}\left[\mathcal{L}_{\ell}\right]=\frac{1}{2 \sqrt{2}} \sqrt{\ell(\ell+1)}
$$

while the asymptotic behaviour of the variance was derived in [Wig10]: as $\ell \rightarrow+\infty$,

$$
\operatorname{Var}\left(\mathcal{L}_{\ell}\right) \sim \frac{1}{32} \log \ell
$$

The second order fluctuations of $\mathcal{L}_{\ell}$ are Gaussian; more precisely, in [MRW17] it was shown that

$$
\frac{\mathcal{L}_{\ell}-\mathbb{E}\left[\mathcal{L}_{\ell}\right]}{\sqrt{\operatorname{Var}\left(\mathcal{L}_{\ell}\right)}} \stackrel{d}{\rightarrow} Z
$$

where $Z$ is a standard Gaussian random variable. 


\section{Outline of the Paper}

2.1. On the proofs of the main results. A well-known consequence of the area/co-area formulae and of the fact that $B_{E}$ is $\mathbb{P}$-a.s. a smooth field, is that one can represent in integral form the nodal length $\mathcal{L}_{E}$ in (1.10) and the number of nodal points $\mathcal{N}_{E}$ in (1.14), respectively, as

$$
\begin{aligned}
\mathcal{L}_{E} & =\int_{\mathcal{D}} \delta_{0}\left(B_{E}(x)\right)\left\|\nabla B_{E}(x)\right\| d x, \\
\mathcal{N}_{E} & =\int_{\mathcal{D}} \delta_{0}\left(B_{E}(x)\right) \delta_{0}\left(\widehat{B}_{E}(x)\right)\left|\operatorname{Jac}_{B_{E}, \widehat{B}_{E}},(x)\right| d x,
\end{aligned}
$$

where $\delta_{0}$ denotes the Dirac mass at $0, \nabla B_{E}$ is the gradient field, and $\mathrm{Jac}_{B_{E}, \widehat{B}_{E}}$ stands for the Jacobian of $\left(B_{E}, \widehat{B}_{E}\right)$ (remember that $\widehat{B}_{E}$ is an independent copy of $B_{E}$ ); on the right-hand sides of (2.23) and (2.24), integrals involving Dirac masses have to be understood as $\mathbb{P}$-a.s. limits of analogous integrals, where $\delta_{0}$ is replaced by an adequate approximation of the identity. We will show in Sect. 3.1 that $\mathcal{L}_{E}$ and $\mathcal{N}_{E}$ are both square-integrable random variables. Combined with (2.23) and (2.24), this will allow us to deploy in Sect. 3.2 the powerful theory of Wiener-Itô chaos expansions (see e.g. [NP12]), yielding that both $\mathcal{L}_{E}$ and $\mathcal{N}_{E}$ admit an explicit representation as orthogonal series, both converging in $L^{2}(\mathbb{P})$, with the form

$$
\mathcal{L}_{E}=\sum_{q=0}^{+\infty} \mathcal{L}_{E}[2 q], \quad \mathcal{N}_{E}=\sum_{q=0}^{+\infty} \mathcal{N}_{E}[2 q],
$$

where $\mathcal{L}_{E}[2 q]$ (resp. $\mathcal{N}_{E}[2 q]$ ) denotes the orthogonal projection of $\mathcal{L}_{E}$ (resp. $\mathcal{N}_{E}$ ) onto the $2 q$ th Wiener chaos associated with $B_{E}$ (and $\widehat{B}_{E}$ ) - - see Sect. 3.2 and [NP12] for definitions and further details. We will see that chaotic decompositions rely in particular on the fact that the sequence of renormalized Hermite polynomials $\left\{H_{q} / \sqrt{q !}\right\}_{q=0,1, \ldots}$ is an orthonormal basis for the space of square-integrable functions on the real line w.r.t. the standard Gaussian density. Note that odd chaoses in (2.25) vanish, since the integrands on the right-hand sides of (2.23) and (2.24) are even.

Our main argument for proving Theorem 1.1 and Theorem 1.4 relies on the investigation of those chaotic components in (2.25) such that $q \geq 1$ (the 0 -th chaotic component is the mean). The second chaotic components $(q=1)$ is investigated in Sect. 4 , where we use the first Green's identity in order to show that $\mathcal{L}_{E}[2]$ and $\mathcal{N}_{E}$ [2] both reduce to a single boundary term, yielding that

$$
\operatorname{Var}\left(\mathcal{L}_{E}[2]\right)=O(1), \quad \operatorname{Var}\left(\mathcal{N}_{E}[2]\right)=O(E) .
$$

The (more difficult) investigation of fourth chaotic components is carried out in Sect. 6: it requires in particular a careful analysis of asymptotic moments of Bessel functions on growing domains, see Sect. 5. Our main finding from Sect. 6 is that

$$
\begin{aligned}
& \operatorname{Var}\left(\mathcal{L}_{E}[4]\right) \sim \operatorname{area}(\mathcal{D}) \frac{1}{512 \pi} \log E \\
& \operatorname{Var}\left(\mathcal{N}_{E}[4]\right) \sim \operatorname{area}(\mathcal{D}) \frac{11}{32 \pi} E \log E .
\end{aligned}
$$


In Sect. 7, we will show that the contribution of higher order chaotic components is negligible, that is: as $E \rightarrow+\infty$,

$$
\begin{aligned}
& \operatorname{Var}\left(\sum_{q \geq 3} \mathcal{L}_{E}[2 q]\right)=o(\log E), \\
& \operatorname{Var}\left(\sum_{q \geq 3} \mathcal{N}_{E}[2 q]\right)=o(E \log E) .
\end{aligned}
$$

This is done by exploiting isotropic property of the field, and by using a Kac-Rice formula to control the second moments of $\mathcal{L}_{E}$ and $\mathcal{N}_{E}$ around the origin.

Substituting (2.26), (2.27) and (2.28) into (2.25), we deduce that the variance of the fourth chaotic component of $\mathcal{L}_{E}$ and $\mathcal{N}_{E}$ is asymptotically equivalent to the corresponding total variances, more precisely: as $E \rightarrow+\infty$,

$$
\begin{aligned}
& \frac{\mathcal{L}_{E}-\mathbb{E}\left[\mathcal{L}_{E}\right]}{\sqrt{\operatorname{Var}\left(\mathcal{L}_{E}\right)}}=\frac{\mathcal{L}_{E}[4]}{\sqrt{\operatorname{Var}\left(\mathcal{L}_{E}[4]\right)}}+o_{\mathbb{P}}(1), \\
& \frac{\mathcal{N}_{E}-\mathbb{E}\left[\mathcal{N}_{E}\right]}{\sqrt{\operatorname{Var}\left(\mathcal{N}_{E}\right)}}=\frac{\mathcal{N}_{E}[4]}{\sqrt{\operatorname{Var}\left(\mathcal{N}_{E}[4]\right)}}+o_{\mathbb{P}}(1),
\end{aligned}
$$

where $o_{\mathbb{P}}(1)$ denotes a sequence converging to zero in probability. Both relations appearing in (2.29), indicate that, in order to conclude the proofs Theorem 1.1 and Theorem 1.4, it is sufficient to check that the normalized projections

$$
\frac{\mathcal{L}_{E}[4]}{\sqrt{\operatorname{Var}\left(\mathcal{L}_{E}[4]\right)}} \text { and } \frac{\mathcal{N}_{E}[4]}{\sqrt{\operatorname{Var}\left(\mathcal{N}_{E}[4]\right)}}
$$

have asymptotically Gaussian fluctuations. Exploiting the fact that both quantities live in a fixed Wiener chaos, this task will be accomplished in Sect. 8, by using techniques of Gaussian analysis taken from [NP12, Chapter 5 and 6], in particular related to the fourth moment theorem from [NuPe05,PT05].

Remark 2.1 (Higher dimensions). It is a challenging and natural question to understand whether the approach adopted in the present paper could be used in order to study the fluctuations of the nodal volume (that is, the volume of the zero set, possibly restricted to a bounded domain) associated with a Gaussian isotropic random wave $X_{d, k}=\left\{X_{d, k}(x)\right.$ : $x \in \mathbb{R}^{d}$ \}, for $d \geq 3$ and in the high-energy limit $k \rightarrow \infty$. Recall that the random field $X_{d, k}$ is by definition the unique (in distribution) unit variance and centered isotropic Gaussian field of $\mathbb{R}^{3}$ almost surely verifying the Helmhotz equation $\Delta X_{d, k}+k^{2} X_{d, k}=0$ on $\mathbb{R}^{3}$. As such, one has necessarily that

$$
\mathbb{E}\left[X_{d, k}(x) X_{d, k}(y)\right]=\frac{J_{(d-2) / 2}(k\|x-y\|)}{(k\|x-y\|)^{(d-2) / 2}}, \quad x \neq y \in \mathbb{R}^{3},
$$

see e.g. [AT, Theorem 5.7.2]. Now, for every $d \geq 3$, it is straightforward to apply the co-area formula in order to deduce an explicit expression of the nodal volume of $X_{d, k}$, analogous to the first relation in (2.25) (see e.g. [Cam17] for a similar analysis involving arithmetic random waves on tori with dimension $d \geq 3$ ). However, some preliminary analysis in this direction has shown us that asymptotic relations analogous to those appearing in (2.26) and (2.29) - involving the second, the fourth, and higher order 
chaotic projections - might fail to hold in dimension $d \geq 3$. In particular, our preliminary computations make it reasonable to conjecture that, when $d \geq 3$, the projection on the second Wiener chaos of the nodal volume of $X_{d, k}$ is not negligible with respect to the total variance. Such a striking phenomenon seems to be related to the special form of the boundary term appearing when applying Green's formula, in a way similar to what is done in the proof of Lemma 4.1 below. Clearly, a complete proof of such a claim is largely outside the scope of the present paper, and is left open for future research. We also observe that the just described boundary effect trivially disappears in the case of arithmetic random waves_-see again [Cam17].

2.2. Further comparison with previous work. The idea of proving limit theorems for nodal quantities of random Laplace eigenfunctions, by first deriving the chaos decompositions (2.25) and then by proving that the fourth chaotic projection is dominating, first appeared in [MPRW16], and has been further developed in the already quoted references [DNPR16, MRW17,PR17, RoW17]. While the techniques adopted in the present paper are directly connected to such a line of research, several crucial differences with previous contributions should be highlighted.

(i) Differently from [MPRW16,DNPR16,MRW17,PR17], the random fields considered in the present paper are eigenfunctions of the Laplace operator on a noncompact manifold (namely, the plane), that one subsequently restricts to a smooth compact domain $\mathcal{D}$. This situation implies in particular that, throughout our proofs and differently from [DNPR16,MPRW16,MRW17,PR17], we cannot exploit any meaningful representation of $B_{E}$ (or $B_{E}^{\mathbb{C}}$ ) in terms of a countable orthogonal basis of Laplace eigenfunctions on $\mathcal{D}$, thus making our computations considerably more delicate. In particular, the representation (1.5) cannot be directly used in our framework. This additional difficulty explains, in particular, the need of developing novel estimates for Bessel functions on growing domains, as derived in Sect. 5.

(ii) Another consequence of the non-compactness of $\mathbb{R}^{2}$ is that (differently from the situation in [MPRW16,DNPR16,PR17]) it is not possible to represent the dominating chaotic projections $\mathcal{L}_{E}$ [4] and $\mathcal{N}_{E}$ [4] as an explicit functional of a finite collection of independent Gaussian coefficients. This implies in particular that, in order to show that $\mathcal{L}_{E}$ [4] and $\mathcal{N}_{E}$ [4] exhibit Gaussian fluctuations, one cannot rely on the usual CLT, but one has rather to apply the analytical techniques based on the use of contractions described in [NP12, Chapter 5]—see Sect. 8.

(iii) Differently from [MPRW16,MRW17], our proof of the variance asymptotic behaviour for nodal quantities (1.12) and (1.16) is done from scratch, and does not make use of previous computations in the literature. In particular, our analysis provides a self-contained rigorous proof of Berry's relations (1.8).

2.3. Plan. In Sect. 3 we derive the chaotic decomposition (2.25) for the nodal length and the number of nodal points. The second chaotic components are investigated in Sect. 4 to obtain (2.26), whereas the main results on asymptotic moments of Bessel functions are in Sect. 5 (further technical results are collected in Appendix B). The fourth chaotic components are studied in Sect. 6 in order to obtain (2.27), and (2.28) is proven in Sect. 7. The Central Limit Theorem for the fourth chaotic component is proved in Sect. 8. Finally, the proof of our main results is given in Sect. 8.2. Additional technical lemmas are gathered together in Appendix A and Appendix C. 


\section{Nodal Statistics and Wiener Chaos}

3.1. Mean square approximation. In order to derive the chaotic decomposition (2.25) for the nodal length and the number of nodal points, we will need the distribution of the random vector $\left(B_{E}(x), B_{E}(y), \nabla B_{E}(x), \nabla B_{E}(y)\right) \in \mathbb{R}^{6}$ for $x, y \in \mathbb{R}^{2}$, where $\nabla B_{E}$ is the gradient field $\nabla:=\left(\partial_{1}, \partial_{2}\right), \partial_{i}:=\partial_{x_{i}}=\partial / \partial x_{i}$ (for $\left.i=1,2\right)$. Let us introduce the following notation: for $i, j \in\{0,1,2\}$

$$
r_{i, j}^{E}(x-y):=\partial_{x_{i}} \partial_{y_{j}} r^{E}(x-y),
$$

with $\partial_{x_{0}}$ and $\partial_{y_{0}}$ equal to the identity by definition. The following result will be proved in Appendix A.

Lemma 3.1. The centered Gaussian vector $\left(B_{E}(x), B_{E}(y), \nabla B_{E}(x), \nabla B_{E}(y)\right) \in \mathbb{R}^{6}$ $\left(x \neq y \in \mathbb{R}^{2}\right)$ has the following covariance matrix:

$$
\Sigma^{E}(x-y)=\left(\begin{array}{cc}
\Sigma_{1}^{E}(x-y) & \Sigma_{2}^{E}(x-y) \\
\Sigma_{2}^{E}(x-y)^{t} & \Sigma_{3}^{E}(x-y)
\end{array}\right)
$$

where

$$
\Sigma_{1}^{E}(x-y)=\left(\begin{array}{cc}
1 & r^{E}(x-y) \\
r^{E}(x-y) & 1
\end{array}\right),
$$

$r^{E}$ being defined in (1.9),

$$
\Sigma_{2}^{E}(x-y)=\left(\begin{array}{cccc}
0 & 0 & r_{0,1}^{E}(x-y) & r_{0,2}^{E}(x-y) \\
-r_{0,1}^{E}(x-y) & -r_{0,2}^{E}(x-y) & 0 & 0
\end{array}\right),
$$

with, for $i=1,2$,

$$
r_{0, i}^{E}(x-y)=2 \pi \sqrt{E} \frac{x_{i}-y_{i}}{\|x-y\|} J_{1}(2 \pi \sqrt{E}\|x-y\|) .
$$

Finally,

$$
\Sigma_{3}^{E}(x-y)=\left(\begin{array}{cccc}
2 \pi^{2} E & 0 & r_{1,1}^{E}(x-y) & r_{1,2}^{E}(x-y) \\
0 & 2 \pi^{2} E & r_{2,1}^{E}(x-y) & r_{2,2}^{E}(x-y) \\
r_{1,1}^{E}(x-y) & r_{2,1}^{E}(x-y) & 2 \pi^{2} E & 0 \\
r_{1,2}^{E}(x-y) & r_{2,2}^{E}(x-y) & 0 & 2 \pi^{2} E
\end{array}\right),
$$

where for $i=1,2$

$$
\begin{aligned}
r_{i, i}^{E}(x-y)= & 2 \pi^{2} E\left(J_{0}(2 \pi \sqrt{E}\|x-y\|)\right. \\
& \left.+\left(1-2 \frac{\left(x_{i}-y_{i}\right)^{2}}{\|x-y\|^{2}}\right) J_{2}(2 \pi \sqrt{E}\|x-y\|)\right),
\end{aligned}
$$

and

$$
\begin{aligned}
& r_{12}^{E}(x-y)=r_{2,1}^{E}(x-y) \\
& \quad=-4 \pi^{2} E \frac{\left(x_{1}-y_{1}\right)\left(x_{2}-y_{2}\right)}{\|x-y\|^{2}} J_{2}(2 \pi \sqrt{E}\|x-y\|) .
\end{aligned}
$$


For brevity, we will sometimes omit the dependence on $x-y$ in the covariance matrix (3.31) just above, as well as in (3.30). In view of Lemma 3.1, we define the normalized derivatives as

$$
\widetilde{\partial}_{i}:=\frac{\partial_{i}}{\sqrt{2 \pi^{2} E}}, \quad i=1,2,
$$

and, accordingly, the normalized gradient $\widetilde{\nabla}$ as

$$
\widetilde{\nabla}:=\left(\widetilde{\partial}_{1}, \widetilde{\partial}_{2}\right)=\frac{\nabla}{\sqrt{2 \pi^{2} E}} .
$$

Let us now consider, for $\varepsilon>0$, the following random variables

$$
\begin{aligned}
\mathcal{L}_{E}^{\varepsilon} & :=\frac{1}{2 \varepsilon} \int_{\mathcal{D}} 1_{[-\varepsilon, \varepsilon]}\left(B_{E}(x)\right)\left\|\nabla B_{E}(x)\right\| d x, \\
\mathcal{N}_{E}^{\varepsilon} & :=\frac{1}{(2 \varepsilon)^{2}} \int_{\mathcal{D}} 1_{[-\varepsilon, \varepsilon]}\left(B_{E}(x)\right) 1_{[-\varepsilon, \varepsilon]}\left(\widehat{B}_{E}(x)\right)\left|\operatorname{Jac}_{B_{E}, \widehat{B}_{E}}(x)\right| d x,
\end{aligned}
$$

where $\mathrm{Jac}_{B_{E}, \widehat{B}_{E}}$ still denotes the Jacobian of $\left(B_{E}, \widehat{B}_{E}\right)$. The random objects in (3.37) and (3.38) can be viewed as $\varepsilon$-approximations of the nodal length of $B_{E}$ in $\mathcal{D}$ and of the number of nodal points of $B_{E}^{\mathbb{C}}$ in $\mathcal{D}$, respectively (here and in what follows, $1_{[-\varepsilon, \varepsilon]}$ denotes the indicator functions of the interval $[-\varepsilon, \varepsilon]$ ). Indeed, the following standard result holds, which will be proved in Appendix A for completeness.

Lemma 3.2. As $\varepsilon \rightarrow 0$,

$$
\mathcal{L}_{E}^{\varepsilon} \stackrel{\text { a.s. }}{\longrightarrow} \mathcal{L}_{E},
$$

where $\mathcal{L}_{E}^{\varepsilon}$ (resp. $\left.\mathcal{L}_{E}\right)$ is given in (3.37) (resp. (1.10)). Moreover,

$$
\mathcal{N}_{E}^{\varepsilon} \stackrel{\text { a.s. }}{\longrightarrow} \mathcal{N}_{E}
$$

where $\mathcal{N}_{E}^{\varepsilon}$ (resp. $\mathcal{N}_{E}$ ) is given in (3.38) (resp. (1.14)).

The next lemma, also proved in Appendix A, shows that the convergence in Lemma 3.2 holds in $L^{2}(\mathbb{P})$.

Lemma 3.3. The nodal length $\mathcal{L}_{E}$ in (1.10) and the number of nodal points $\mathcal{N}_{E}(1.14)$ are finite-variance random variables, and both convergences in (3.39) and (3.40) hold in $L^{2}(\mathbb{P})$, i.e. as $\varepsilon \rightarrow 0$,

$$
\begin{aligned}
& \mathbb{E}\left[\left|\mathcal{L}_{E}^{\varepsilon}-\mathcal{L}_{E}\right|^{2}\right] \rightarrow 0, \\
& \mathbb{E}\left[\left|\mathcal{N}_{E}^{\varepsilon}-\mathcal{N}_{E}\right|^{2}\right] \rightarrow 0 .
\end{aligned}
$$


3.2. Chaotic expansions. We start by observing that the field $B_{E}$ can be represented as a Wiener-Itô integral as follows:

$$
B_{E}(x)=\frac{1}{\sqrt{2 \pi}} \int_{\mathbb{S}^{1}} \mathrm{e}^{2 \pi \sqrt{E} i\langle\theta, x\rangle} d G(\theta), \quad x \in \mathbb{R}^{2},
$$

where $G$ is a complex Hermitian Gaussian measure on the unit circle $\mathbb{S}^{1}$ with Lebesgue control measure (see $[\mathrm{NP} 12, \S 2.1]$ and in particular Example 2.1.4). By the integral representation [AS64, §9.1] of Bessel functions,

$$
\mathbb{E}\left[B_{E}(x) B_{E}(y)\right]=\frac{1}{2 \pi} \int_{\mathbb{S}^{1}} \mathrm{e}^{2 \pi \sqrt{E} i\langle\theta, x-y\rangle} d \theta=r^{E}(x-y), \quad x, y \in \mathbb{R}^{2} .
$$

Remark 3.4. We will sometimes prefer to represent such quantities as $B_{E}(x), \partial_{1} B_{E}(x)$ (and so on) as stochastic integrals of deterministic kernels with respect to a real-valued Gaussian measure (and not a complex-valued one, as in (3.43) — this is alway possible, due to standard properties of separable real Hilbert spaces). See e.g. Sect. 8, where such a representation is implicitly used for dealing with contraction operators.

The random variables $\mathcal{L}_{E}^{\varepsilon}$ and $\mathcal{N}_{E}^{\varepsilon}$ having finite variance (Lemma 3.3) functionals of $B_{E}$ in (3.43), they admit a so-called chaotic expansion [NP12, §2.2], i.e. they can be written as a random orthogonal series

$$
\mathcal{L}_{E}^{\varepsilon}=\sum_{q=0}^{+\infty} \mathcal{L}_{E}^{\varepsilon}[q], \quad \mathcal{N}_{E}^{\varepsilon}=\sum_{q=0}^{+\infty} \mathcal{N}_{E}^{\varepsilon}[q],
$$

converging in $L^{2}$. The term $\mathcal{L}_{E}^{\varepsilon}[q]$ (resp. $\left.\mathcal{N}_{E}^{\varepsilon}[q]\right)$ is the orthogonal projection of $\mathcal{L}_{E}^{\varepsilon}$ (resp. $\mathcal{N}_{E}^{\varepsilon}$ ) onto the so-called $q$ th Wiener chaos $C_{q}$ [NP12, Definition 2.2.3]. The definition of the latter involves the sequence of Hermite polynomials $\left\{H_{n}\right\}_{n \geq 0}$ [NP12, Definition 1.4.1] which are a complete orthonormal basis (up to normalization) of the space of square integrable functions on the real line w.r.t. the standard Gaussian density. We recall here the expression of the first Hermite polynomials:

$$
\begin{aligned}
& H_{0}(t)=1, H_{1}(t)=t, H_{2}(t)=t^{2}-1, \\
& H_{3}(t)=t^{3}-3 t, H_{4}(t)=t^{4}-6 t^{2}+3
\end{aligned}
$$

We recall also that, for normalized $Z_{1}, Z_{2}$ jointly Gaussian, we have for any $n, n^{\prime} \in$ $\{0,1,2, \ldots\}$

$$
\mathbb{E}\left[H_{n}\left(Z_{1}\right) H_{n^{\prime}}\left(Z_{2}\right)\right]=\delta_{n}^{n^{\prime}} n ! \mathbb{E}\left[Z_{1} Z_{2}\right]^{n} .
$$

In view of (3.47) and Lemma 3.1, we rewrite (3.37) and (3.38) as

$$
\begin{aligned}
\mathcal{L}_{E}^{\varepsilon} & =\frac{\sqrt{2 \pi^{2} E}}{2 \varepsilon} \int_{\mathcal{D}} 1_{[-\varepsilon, \varepsilon]}\left(B_{E}(x)\right)\left\|\widetilde{\nabla} B_{E}(x)\right\| d x, \\
\mathcal{N}_{E}^{\varepsilon} & =\frac{2 \pi^{2} E}{(2 \varepsilon)^{2}} \int_{\mathcal{D}} 1_{[-\varepsilon, \varepsilon]}\left(B_{E}(x)\right) 1_{[-\varepsilon, \varepsilon]}\left(\widehat{B}_{E}(x)\right)\left|\widetilde{\mathrm{Jac}}_{B_{E}, \widehat{B}_{E}}(x)\right| d x,
\end{aligned}
$$

where $\widetilde{\nabla}$ is the normalized gradient (3.36), and $\widetilde{J a c}_{B_{E}, \widehat{B}_{E}}$ denotes the Jacobian of $\left(B_{E}, \widehat{B}_{E}\right)$ w.r.t. the normalized derivatives (3.35).

The chaotic expansion for $\mathcal{L}_{E}^{\varepsilon}\left(\right.$ resp. $\mathcal{N}_{E}^{\varepsilon}$ ) can be obtained as in [MPRW16, Lemma 3.4, Lemma 3.5] (resp. as in the proof of [DNPR16, Lemma 4.4]) (the terms corresponding to odd chaoses vanish, due to the parity of integrand functions in (3.37) and (3.38)). The proof of the following result is hence omitted. 
Lemma 3.5. The chaotic components of $\mathcal{L}_{E}^{\varepsilon}$ in (3.48) corresponding to odd chaoses vanish, i.e.

$$
\mathcal{L}_{E}^{\varepsilon}[2 q+1]=0, \quad q \geq 0,
$$

while for even chaoses

$$
\begin{aligned}
\mathcal{L}_{E}^{\varepsilon}[2 q]= & \sqrt{2 \pi^{2} E} \sum_{u=0}^{q} \sum_{m=0}^{u} \beta_{2 q-2 u}^{\varepsilon} \alpha_{2 m, 2 u-2 m} \\
& \times \int_{\mathcal{D}} H_{2 q-2 u}\left(B_{E}(x)\right) H_{2 m}\left(\widetilde{\partial}_{1} B_{E}(x)\right) H_{2 u-2 m}\left(\widetilde{\partial}_{2} B_{E}(x)\right) d x,
\end{aligned}
$$

where $\left\{\beta_{2 n}^{\varepsilon}\right\}_{n \geq 0}$ is the sequence of chaotic coefficients of $\frac{1}{2 \varepsilon} 1_{[-\varepsilon, \varepsilon]}$ appearing in [MPRW16, Lemma 3.4], while $\left\{\alpha_{2 n, 2 m}\right\}_{n, m \geq 0}$ is the sequence of chaotic coeffients of the Euclidean norm in $\mathbb{R}^{2}\|\cdot\|$ appearing in [MPRW16, Lemma 3.5].

The chaotic components of $\mathcal{N}_{E}^{\varepsilon}$ in (3.49) are

$$
\mathcal{N}_{E}^{\varepsilon}[2 q+1]=0, \quad q \geq 0,
$$

while for even chaoses

$$
\begin{aligned}
\mathcal{N}_{E}^{\varepsilon}[2 q]= & 2 \pi^{2} E \sum_{i_{1}+i_{2}+i_{3}+j_{1}+j_{2}+j_{3}=q} \beta_{i_{1}}^{\varepsilon} \beta_{j_{1}}^{\varepsilon} \gamma_{i_{2}, i_{3}, j_{2}, j_{3}} \\
& \times \int_{\mathcal{D}} H_{i_{1}}\left(B_{E}(x)\right) H_{i_{1}}\left(\widehat{B}_{E}(x)\right) H_{i_{2}}\left(\widetilde{\partial}_{1} B_{E}(x)\right) \\
& \times H_{i_{3}}\left(\widetilde{\partial}_{2} B_{E}(x)\right) H_{i_{2}}\left(\widetilde{\partial}_{1} \widehat{B}_{E}(x)\right) H_{i_{3}}\left(\widetilde{\partial}_{2} \widehat{B}_{E}(x)\right) d x,
\end{aligned}
$$

where $i_{1}, j_{1}$ are even, and $i_{2}, i_{3}, j_{2}, j_{3}$ have the same parity; here the sequence $\left\{\gamma_{i_{2}, i_{3}, j_{2}, j_{3}}\right\}$ corresponds to the chaotic expansion of the absolute value of the Jacobian appearing in [DNPR16, Lemma 4.2].

Let us define, as in [MPRW16, Lemma 3.4],

$$
\beta_{2 n}:=\lim _{\varepsilon \rightarrow 0} \beta_{2 n}^{\varepsilon} .
$$

The sequence $\left\{\beta_{2 n}\right\}_{n \geq 0}$ consists of the (formal) chaotic coefficients of the Dirac mass $\delta_{0}$. Hence from Lemmas 3.3 and 3.5 we immediately obtain the chaotic expansions for $\mathcal{L}_{E}$ and $\mathcal{N}_{E}$.

Proposition 3.6. The chaotic expansion of the nodal length in $\mathcal{D}$ is

$$
\begin{aligned}
\mathcal{L}_{E}= & \sum_{q=0}^{+\infty} \mathcal{L}_{E}[2 q]=\sqrt{2 \pi^{2} E} \sum_{q=0}^{+\infty} \sum_{u=0}^{q} \sum_{m=0}^{u} \beta_{2 q-2 u} \alpha_{2 m, 2 u-2 m} \\
& \times \int_{\mathcal{D}} H_{2 q-2 u}\left(B_{E}(x)\right) H_{2 m}\left(\widetilde{\partial}_{1} B_{E}(x)\right) H_{2 u-2 m}\left(\widetilde{\partial}_{2} B_{E}(x)\right) d x,
\end{aligned}
$$

where $\left\{\beta_{2 n}\right\}_{n \geq 0}$ is defined in (3.50) (see also [MPRW16, Lemma 3.4]), while $\left\{\alpha_{2 n, 2 m}\right\}_{n, m \geq 0}$ is the sequence of chaotic coeffients of the Euclidean norm in $\mathbb{R}^{2}\|\cdot\|$ appearing in [MPRW16, Lemma 3.5]. 
For the number of phase singularities in $\mathcal{D}$ we have

$$
\begin{aligned}
\mathcal{N}_{E}= & \sum_{q=0}^{+\infty} \mathcal{N}_{E}[2 q]=2 \pi^{2} E \sum_{q=0}^{+\infty} \sum_{i_{1}+i_{2}+i_{3}+j_{1}+j_{2}+j_{3}=q} \beta_{i_{1}} \beta_{j_{1}} \gamma_{i_{2}, i_{3}, j_{2}, j_{3}} \\
& \times \int_{\mathcal{D}} H_{i_{1}}\left(B_{E}(x)\right) H_{i_{1}}\left(\widehat{B}_{E}(x)\right) H_{i_{2}}\left(\widetilde{\partial}_{1} B_{E}(x)\right) \\
& \times H_{i_{3}}\left(\widetilde{\partial}_{2} B_{E}(x)\right) H_{i_{2}}\left(\widetilde{\partial}_{1} \widehat{B}_{E}(x)\right) H_{i_{3}}\left(\widetilde{\partial}_{2} \widehat{B}_{E}(x)\right) d x,
\end{aligned}
$$

where $i_{1}, j_{1}$ are even, and $i_{2}, i_{3}, j_{2}, j_{3}$ have the same parity; here the sequence $\left\{\gamma_{i_{2}, i_{3}, j_{2}, j_{3}}\right\}$ corresponds to the chaotic expansion of the absolute value of the Jacobian appearing in [DNPR16, Lemma 4.2].

We will need the explicit values of few chaotic coefficients for $\mathcal{L}_{E}$ and $\mathcal{N}_{E}$ (see [DNPR16, Lemma 4.3] and the proofs of [MPRW16, Proposition 3.2] and [MPRW16, Lemma 4.2]):

$$
\beta_{0}=\frac{1}{\sqrt{2 \pi}}, \quad \beta_{2}=-\frac{1}{2 \sqrt{2 \pi}}, \quad \beta_{4}=\frac{1}{8 \sqrt{2 \pi}}
$$

and

$$
\begin{aligned}
& \alpha_{0,0}=\frac{\sqrt{2 \pi}}{2}, \quad \alpha_{2,0}=\alpha_{0,2}=\frac{\sqrt{2 \pi}}{8}, \\
& \alpha_{4,0}=\alpha_{0,4}=-\frac{\sqrt{2 \pi}}{128}, \quad \alpha_{2,2}=-\frac{\sqrt{2 \pi}}{64},
\end{aligned}
$$

finally

$$
\begin{aligned}
& \gamma_{0,0,0,0}=1, \quad \gamma_{2,0,0,0}=\gamma_{0,2,0,0}=\gamma_{0,0,2,0}=\gamma_{0,0,0,2}=\frac{1}{4}, \\
& \gamma_{1,1,1,1}=-\frac{3}{8}, \quad \gamma_{2,2,0,0}=\gamma_{0,0,2,2}=-\frac{1}{32}, \\
& \gamma_{2,0,2,0}=\gamma_{0,2,0,2}=-\frac{1}{32}, \quad \gamma_{2,0,0,2}=\gamma_{0,2,2,0}=\frac{5}{32}, \\
& \gamma_{4,0,0,0}=\gamma_{0,4,0,0}=\gamma_{0,0,4,0}=\gamma_{0,0,0,4}=-\frac{3}{192} .
\end{aligned}
$$

\section{Second Chaotic Components}

In this section we investigate the second chaotic component of the nodal length and the number of nodal components, respectively.

Lemma 4.1. For the second chaotic component of $\mathcal{L}_{E}$ we have

$$
\mathcal{L}_{E}[2]=\frac{1}{8 \pi \sqrt{2 E}} \int_{\partial \mathcal{D}} B_{E}(x)\left\langle\nabla B_{E}(x), n(x)\right\rangle d x,
$$

where $n(x)$ is the outward pointing normal at $x$, hence

$$
\operatorname{Var}\left(\mathcal{L}_{E}[2]\right)=O(1) .
$$


Proof. Equation (3.51) implies that the projection $\mathcal{L}_{E}$ [2] of $\mathcal{L}_{E}$ onto the second chaos is given by

$$
\begin{aligned}
\mathcal{L}_{E}[2]= & \sqrt{2 \pi^{2} E}\left\{\beta_{2} \alpha_{0,0} \int_{\mathcal{D}} H_{2}\left(B_{E}(x)\right) d x+\beta_{0} \alpha_{0,2} \int_{\mathcal{D}} H_{2}\left(\widetilde{\partial}_{1} B_{E}(x)\right) d x\right. \\
& \left.+\beta_{0} \alpha_{2}, \int_{\mathcal{D}} H_{2}\left(\widetilde{\partial}_{2} B_{E}(x)\right) d x\right\} \\
= & \frac{\pi}{8} \sqrt{2 E}\left\{-2 \int_{\mathcal{D}} B_{E}(x)^{2} d x+\int_{\mathcal{D}}\left\|\widetilde{\nabla} B_{E}(x)\right\|^{2} d x\right\},
\end{aligned}
$$

where we used the explicit expression of the second Hermite polynomial (3.46). The first Green identity [Lee97, p. 44] (see also [Ros15, Proposition 7.3.1] and the proof of [DNPR16, Lemma 4.4]) asserts that

$$
\int_{\mathcal{D}}\left\|\nabla B_{E}(x)\right\|^{2} d x=-\int_{\mathcal{D}} B_{E}(x) \Delta B_{E}(x) d x+\int_{\partial \mathcal{D}} B_{E}(x)\left\langle\nabla B_{E}(x), n(x)\right\rangle d x
$$

where $n(x)$ denotes the outward pointing unit normal at $x$. As a result,

$$
\begin{aligned}
\int_{\mathcal{D}}\left\|\widetilde{\nabla} B_{E}(x)\right\|^{2} d x & =\frac{1}{2 \pi^{2} E} \int_{\mathcal{D}}\left\|\nabla B_{E}(x)\right\|^{2} d x \\
& =2 \int_{\mathcal{D}} B_{E}(x)^{2} d x+\frac{1}{2 \pi^{2} E} \int_{\partial \mathcal{D}} B_{E}(x)\left\langle\nabla B_{E}(x), n(x)\right\rangle d x,
\end{aligned}
$$

implying in turn from (4.58) that

$$
\mathcal{L}_{E}[2]=\frac{1}{8 \pi \sqrt{2 E}} \int_{\partial \mathcal{D}} B_{E}(x)\left\langle\nabla B_{E}(x), n(x)\right\rangle d x,
$$

which is (4.56). From (4.59) we deduce (4.57), indeed,

$$
\begin{aligned}
\operatorname{Var}\left(\mathcal{L}_{E}[2]\right) & \leq \frac{1}{128 \pi^{2} E} \int_{\partial \mathcal{D}} \mathbb{E}\left[B_{E}(x)^{2}\right] d x \cdot \int_{\partial \mathcal{D}} \mathbb{E}\left[\left\|\nabla B_{E}(x)\right\|^{2}\right] d x \\
& =\frac{1}{64} \text { perimeter }(\mathcal{D})^{2}=O(1) .
\end{aligned}
$$

Lemma 4.2. For the second chaotic component of $\mathcal{N}_{E}$ we have

$$
\mathcal{N}_{E}[2]=\sqrt{2 E}\left(\mathcal{L}_{E}[2]+\widehat{\mathcal{L}}_{E}[2]\right)
$$

(with obvious notation), hence

$$
\operatorname{Var}\left(\mathcal{N}_{E}[2]\right)=O(E) .
$$

Proof. Similarly to (4.58), from (3.52) we have

$$
\begin{aligned}
\mathcal{N}_{E}[2]= & 2 \pi^{2} E\left\{\beta_{2} \beta_{0} \gamma_{0,0,0,0} \int_{\mathcal{D}} H_{2}\left(B_{E}(x)\right) d x+\beta_{0} \beta_{2} \gamma_{0,0,0,0} \int_{\mathcal{D}} H_{2}\left(\widehat{B}_{E}(x)\right) d x\right. \\
& +\beta_{0}^{2} \gamma_{2,0,0,0} \int_{\mathcal{D}} H_{2}\left(\widetilde{\partial}_{1} B_{E}(x)\right) d x+\beta_{0}^{2} \gamma_{0,2,0,0} \int_{\mathcal{D}} H_{2}\left(\widetilde{\partial}_{2} B_{E}(x)\right) d x
\end{aligned}
$$




$$
\begin{aligned}
& \left.+\beta_{0}^{2} \gamma_{0,0,2,0} \int_{\mathcal{D}} H_{2}\left(\widetilde{\partial}_{1} \widehat{B}_{E}(x)\right) d x+\beta_{0}^{2} \gamma_{0,0,0,2} \int_{\mathcal{D}} H_{2}\left(\widetilde{\partial}_{2} \widehat{B}_{E}(x)\right) d x\right\} \\
= & \frac{\pi E}{4}\left\{-2 \int_{\mathcal{D}} B_{E}(x)^{2} d x+\int_{\mathcal{D}}\left\|\widetilde{\nabla} B_{E}(x)\right\|^{2} d x\right. \\
& \left.-2 \int_{\mathcal{D}} \widehat{B}_{E}(x)^{2} d x+\int_{\mathcal{D}}\left\|\widetilde{\nabla} \widehat{B}_{E}(x)\right\|^{2} d x\right\} .
\end{aligned}
$$

That is, $\mathcal{N}_{E}[2]=\sqrt{2 E}\left(\mathcal{L}_{E}[2]+\widehat{\mathcal{L}}_{E}[2]\right)$ (4.60), implying in turn (4.61) (cf. (4.57))

$$
\operatorname{Var}\left(\mathcal{N}_{E}[2]\right)=\frac{E}{16} \operatorname{perimeter}(\mathcal{D})^{2}=O(E) .
$$

\section{Moments of Bessel Functions}

In order to investigate the fourth chaotic components of $\mathcal{L}_{E}$ and $\mathcal{N}_{E}$, we first need a technical result on moments of Bessel functions on convex bodies.

Let us define (cf. (3.30)), for $k, l \in\{0,1,2\}$,

$$
\widetilde{r}_{k, l}^{E}(x, y)=\widetilde{r}_{k, l}^{E}(x-y):=\mathbb{E}\left[\widetilde{\partial}_{k} B_{E}(x) \widetilde{\partial}_{l} B_{E}(y)\right], \quad x, y \in \mathbb{R}^{2},
$$

with $\widetilde{\partial}_{0} B_{E}:=B_{E}$. Note that $\widetilde{r}_{0,0}^{E} \equiv r^{E}$.

Since for $n=0,1,2$,

$$
J_{n}(\psi)=O\left(\frac{1}{\sqrt{\psi}}\right)
$$

uniformly for $\psi \in[0,+\infty$ ) (see [Sze75]), from Lemma 3.1 we have that for every $k, l \in\{0,1,2\}$,

$$
\widetilde{r}_{k, l}^{E}(z)=O\left(\frac{1}{\sqrt{\sqrt{E}\|z\|}}\right)
$$

uniformly on $z$ and $E$. Now let $(\phi, \theta)$ be standard polar coordinates on $\mathbb{R}^{2}(\phi \in$ $[0,+\infty), \theta \in[0,2 \pi])$. From Lemma 3.1 we have

$$
\begin{aligned}
& \widetilde{r}_{0,1}^{E}((\phi \cos \theta, \phi \sin \theta))=\cos \theta J_{1}(2 \pi \sqrt{E} \phi), \\
& \widetilde{r}_{0,2}^{E}((\phi \cos \theta, \phi \sin \theta))=\sin \theta J_{1}(2 \pi \sqrt{E} \phi),
\end{aligned}
$$

and $\widetilde{r}_{i, 0}^{E}=-\widetilde{r}_{0, i}^{E}$ for $i=1,2$. Moreover

$$
\begin{aligned}
& \tilde{r}_{1,1}^{E}((\phi \cos \theta, \phi \sin \theta))=\left(J_{0}(2 \pi \sqrt{E} \phi)+\left(1-2 \cos ^{2} \theta\right) J_{2}(2 \pi \sqrt{E} \phi)\right), \\
& \tilde{r}_{2,2}^{E}((\phi \cos \theta, \phi \sin \theta))=\left(J_{0}(2 \pi \sqrt{E} \phi)+\left(1-2 \sin ^{2} \theta\right) J_{2}(2 \pi \sqrt{E} \phi)\right) .
\end{aligned}
$$


Finally

$$
\widetilde{r}_{1,2}^{E}((\phi \cos \theta, \phi \sin \theta))=-2 \cos \theta \cdot \sin \theta J_{2}(2 \pi \sqrt{E} \phi)=\widetilde{r}_{2,1}^{E}((\phi \cos \theta, \phi \sin \theta)) .
$$

Recall now that the diameter of $\mathcal{D}$ is defined as

$$
\operatorname{diam}(\mathcal{D}):=\sup _{x, y \in \mathcal{D}}\|x-y\|,
$$

while its inner radius is

$$
\operatorname{inrad}(\mathcal{D}):=\sup \left\{r>0: \exists x \in \mathcal{D} \text { s.t. } B_{r}(x) \subseteq \mathcal{D}\right\} .
$$

As briefly anticipated above, the next two propositions contain key results to investigate the asymptotic behavior of fourth order chaotic components variances in Sect. 6, in particular for the proofs of Lemmas 8.5-8.19 which are collected in Appendix B. We observe that the proof of Proposition 5.1 below can be simplified when $\mathcal{D}$ equals a disk of positive radius.

Proposition 5.1. Let $q_{i, j} \geq 0$ for $i, j=0,1,2$ and $\sum_{i, j=0}^{2} q_{i, j}=4$. Then

$$
\begin{aligned}
& \int_{\mathcal{D}} \int_{\mathcal{D}} \prod_{i, j=0}^{2} \tilde{r}_{i, j}^{E}(x-y)^{q_{i, j}} d x d y \\
& =\operatorname{area}(\mathcal{D}) \int_{0}^{\operatorname{diam}(\mathcal{D})} \phi d \phi \int_{0}^{2 \pi} d \theta \prod_{i, j=0}^{2} \widetilde{r}_{i, j}^{E}((\phi \cos \theta, \phi \sin \theta))^{q_{i, j}}+O\left(\frac{1}{E}\right) .
\end{aligned}
$$

Proof. By the co-area formula we can rewrite the 1.h.s. of (5.64) as

$$
\begin{aligned}
& E \int_{\mathcal{D}} \int_{\mathcal{D}} \prod_{i, j=0}^{2} \widetilde{r}_{i, j}^{E}(x-y)^{q_{i, j}} d x d y \\
& =\int_{0}^{\operatorname{diam}(\mathcal{D})} d \phi \underbrace{\int_{\mathcal{D}} d x \int_{\partial B_{\phi}(x) \cap \mathcal{D}} d y \prod_{i, j=0}^{2} \widetilde{r}_{i, j}^{E}(x-y)^{q_{i, j}}}_{=: f(\phi)},
\end{aligned}
$$

where $B_{\phi}(x)=\{y:\|x-y\| \leq \phi\}$, while $\partial B_{\phi}(x)$ denotes its boundary. For $\phi \in$ $[0, \operatorname{inrad}(\mathcal{D}))$, define

$$
\mathcal{D}_{\phi}:=\left\{x \in \mathcal{D}: B_{\phi}(x) \subseteq \mathcal{D}\right\}
$$

then

$$
\begin{aligned}
f(\phi):= & \int_{\mathcal{D}_{\phi}} d x \int_{\partial B_{\phi}(x)} d y \prod_{i, j=0}^{2} \widetilde{r}_{i, j}^{E}(x-y)^{q_{i, j}} \\
& +\int_{\mathcal{D} \backslash \mathcal{D}_{\phi}} d x \int_{\partial B_{\phi}(x) \cap \mathcal{D}} d y \prod_{i, j=0}^{2} \widetilde{r}_{i, j}^{E}(x-y)^{q_{i, j}} .
\end{aligned}
$$


Using polar coordinates on $\partial B_{\phi}(x)$ we can rewrite the first term of the r.h.s. of (5.65) as

$$
\begin{aligned}
& \int_{\mathcal{D}_{\phi}} d x \int_{\partial B_{\phi}(x)} d y \prod_{i, j=0}^{2} \widetilde{r}_{i, j}^{E}(x-y)^{q_{i, j}} \\
& =\operatorname{area}\left(\mathcal{D}_{\phi}\right) \int_{0}^{2 \pi} \prod_{i, j=0}^{2} \widetilde{r}_{i, j}^{E}((\phi \cos \theta, \phi \sin \theta))^{q_{i, j}} \phi d \theta .
\end{aligned}
$$

We have

$$
\operatorname{area}\left(\mathcal{D}_{\phi}\right)=\operatorname{area}(\mathcal{D})-\operatorname{area}\left(\mathcal{D} \backslash \mathcal{D}_{\phi}\right)
$$

Now since $\mathcal{D} \subseteq \mathcal{D}_{\phi}+2 \phi B_{1}$, where $B_{1}=B_{1}(0)$ denotes the open ball of radius 1 centered at 0 ,

$$
\begin{aligned}
\operatorname{area}\left(\mathcal{D} \backslash \mathcal{D}_{\phi}\right) & \leq \operatorname{area}\left(\mathcal{D}_{\phi}+2 \phi B_{1}\right)-\operatorname{area}\left(\mathcal{D}_{\phi}\right) \\
& =4 W_{1}\left(\mathcal{D}_{\phi}\right) \phi+4 W_{2}\left(\mathcal{D}_{\phi}\right) \phi^{2}
\end{aligned}
$$

where for the last equality we used Steiner formula (for a convex body $K \subseteq \mathbb{R}^{2}$ and $j=$ $0,1,2, W_{j}(K)$ is the $j$ th quermassintegrals) and the equality $W_{0}\left(\mathcal{D}_{\psi}\right)=\operatorname{meas}\left(\mathcal{D}_{\psi}\right)$. Bearing in mind that if $K \subseteq K^{\prime}$, then $W_{j}(K) \leq W_{j}\left(K^{\prime}\right)$ for $j=0,1,2$ we find

$$
\operatorname{area}\left(\mathcal{D} \backslash \mathcal{D}_{\phi}\right) \leq 4 W_{1}(\mathcal{D}) \phi+4 W_{2}(\mathcal{D}) \phi^{2}
$$

Hence we find that

$$
\operatorname{area}\left(\mathcal{D} \backslash \mathcal{D}_{\phi}\right) \int_{0}^{2 \pi} \prod_{i, j=0}^{2} \tilde{r}_{i, j}^{E}((\phi \cos \theta, \phi \sin \theta))^{q_{i, j}} \phi d \theta=O\left(\frac{1}{E}\right)
$$

uniformly for $\phi \in[0$, inrad $(\mathcal{D})$ by using (5.68) and (5.63). Therefore from (5.66) we can write

$$
\begin{aligned}
& \int_{\mathcal{D}_{\phi}} d x \int_{\partial B_{\phi}(x)} d y \prod_{i, j=0}^{2} \widetilde{r}_{i, j}^{E}(x-y)^{q_{i, j}} \\
& =\operatorname{area}(\mathcal{D}) \int_{0}^{2 \pi} \prod_{i, j=0}^{2} \widetilde{r}_{i, j}^{E}((\phi \cos \theta, \phi \sin \theta))^{q_{i, j}} \phi d \theta+O\left(\frac{1}{E}\right) .
\end{aligned}
$$

The error term in (5.65) can be dealt with as before obtaining

$$
\begin{gathered}
\int_{\mathcal{D} \backslash \mathcal{D}_{\phi}} d x \int_{\partial B_{\phi}(x) \cap \mathcal{D}} d y \prod_{i, j=0}^{2}\left|\widetilde{r}_{i, j}^{E}(x-y)\right|^{q_{i, j}} \\
\leq \int_{\mathcal{D} \backslash \mathcal{D}_{\phi}} d x \int_{\partial B_{\phi}(x)} d y \prod_{i, j=0}^{2}\left|\widetilde{r}_{i, j}^{E}(x-y)\right|^{q_{i, j}} \\
=O\left(\operatorname{area}\left(\mathcal{D} \backslash \mathcal{D}_{\phi}\right) \cdot \phi \cdot \frac{1}{E \phi^{2}}\right)=O\left(\frac{1}{E}\right) .
\end{gathered}
$$


Let us now consider $\phi \in[0, \operatorname{diam}(\mathcal{D}))$, then

$$
\begin{aligned}
f(\phi) & =f(\phi) 1_{[0, \operatorname{inrad}(\mathcal{D}))}(\phi)+f(\phi) 1_{[\operatorname{inrad}(\mathcal{D}), \operatorname{diam}(\mathcal{D})}(\phi) \\
& =\operatorname{area}(\mathcal{D}) \int_{0}^{2 \pi} d \theta \prod_{i, j=0}^{2} \widetilde{r}_{i, j}^{E}((\phi \cos \theta, \phi \sin \theta))^{q_{i, j}} \phi \\
& \times 1_{[0, \operatorname{inrad}(\mathcal{D}))}(\phi)+O\left(\frac{1}{E}\right)+f(\phi) 1_{[\operatorname{inrad}(\mathcal{D}), \operatorname{diam}(\mathcal{D})}(\phi) \\
& =\operatorname{area}(\mathcal{D}) \int_{0}^{2 \pi} d \theta \prod_{i, j=0}^{2} \widetilde{r}_{i, j}^{E}((\phi \cos \theta, \phi \sin \theta))^{q_{i, j}} \phi \cdot 1_{[0, \operatorname{diam}(\mathcal{D}))}(\phi) \\
& +\left(f(\phi)-\operatorname{area}(\mathcal{D}) \int_{0}^{2 \pi} d \theta \prod_{i, j=0}^{2} \widetilde{r}_{i, j}^{E}((\phi \cos \theta, \phi \sin \theta))^{q_{i, j} \phi}\right) \\
& \times 1_{[\operatorname{inrad}(\mathcal{D}), \operatorname{diam}(\mathcal{D})}(\phi)+O\left(\frac{1}{E}\right) .
\end{aligned}
$$

Now it suffices to note that

$$
\begin{aligned}
& \left|f(\phi)-\operatorname{area}(\mathcal{D}) \int_{0}^{2 \pi} d \theta \prod_{i, j=0}^{2} \widetilde{r}_{i, j}^{E}((\phi \cos \theta, \phi \sin \theta))^{q_{i, j}} \phi\right| 1_{[\operatorname{inrad}(\mathcal{D}), \operatorname{diam}(\mathcal{D})}(\phi) \\
& \leq 2 \operatorname{area}(\mathcal{D}) \int_{0}^{2 \pi} d \theta \prod_{i, j=0}^{2}\left|\widetilde{r}_{i, j}^{E}((\phi \cos \theta, \phi \sin \theta))\right|^{q_{i, j}} \phi \cdot 1_{[\operatorname{inrad}(\mathcal{D}), \operatorname{diam}(\mathcal{D})}(\phi) \\
& \leq 2 \operatorname{area}(\mathcal{D}) \int_{0}^{2 \pi} d \theta \prod_{i, j=0}^{2}\left|\widetilde{r}_{i, j}^{E}((\phi \cos \theta, \phi \sin \theta))\right|^{q_{i, j}} \frac{\phi^{2}}{\operatorname{inrad}(\mathcal{D})} .
\end{aligned}
$$

In order to study the asymptotic behavior, as $E \rightarrow+\infty$, of (5.64), we need the following uniform estimate for Bessel functions [Kra14, (7)]: for $\alpha \geq-1 / 2$

$$
\frac{1}{\sqrt{2 \pi}} \mu \leq \sup _{x \geq 0} x^{3 / 2}\left|J_{\alpha}(x)-\sqrt{\frac{2}{\pi x}} \cos \left(x-\omega_{\alpha}\right)\right|<\frac{4}{5} \mu,
$$

where $\mu:=\left|\alpha^{2}-1 / 4\right|$ and $\omega_{\alpha}:=(2 \alpha+1) \pi / 4$. From (5.69) we find

$$
\begin{aligned}
& r^{E}((\phi \cos \theta, \phi \sin \theta))=\underbrace{\frac{1}{\pi \sqrt{\sqrt{E} \phi}} \cos \left(2 \pi \sqrt{E} \phi-\frac{\pi}{4}\right)}_{=: h^{E}(\theta) g^{E}(\phi)}+O\left(\frac{1}{E^{3 / 4} \phi \sqrt{\phi}}\right) \\
& \widetilde{r}_{0,1}^{E}((\phi \cos \theta, \phi \sin \theta))=\underbrace{\frac{\sqrt{2} \cos \theta}{\pi \sqrt{\sqrt{E} \phi}} \sin \left(2 \pi \sqrt{E} \phi-\frac{\pi}{4}\right)}_{=: h_{0,1}^{E}(\theta) g_{0,1}^{E}(\phi)}+O\left(\frac{1}{E^{3 / 4} \phi \sqrt{\phi}}\right)
\end{aligned}
$$




$$
\begin{aligned}
& \tilde{r}_{0,2}^{E}((\phi \cos \theta, \phi \sin \theta))=\underbrace{\frac{\sqrt{2} \sin \theta}{\pi \sqrt{\sqrt{E} \phi} \sin \left(2 \pi \sqrt{E} \phi-\frac{\pi}{4}\right)}+O\left(\frac{1}{E^{3 / 4} \phi \sqrt{\phi}}\right)}_{=: h_{0,2}^{E}(\theta) g_{0,2}^{E}(\phi)} \\
& \tilde{r}_{1,1}^{E}((\phi \cos \theta, \phi \sin \theta))=\underbrace{\frac{2 \cos ^{2} \theta}{\pi \sqrt{\sqrt{E} \phi}} \cos \left(2 \pi \sqrt{E} \phi-\frac{\pi}{4}\right)}_{=: h_{1,1}^{E}(\theta) g_{1,1}^{E}(\phi)}+O\left(\frac{1}{E^{3 / 4} \phi \sqrt{\phi}}\right) \\
& \tilde{r}_{2,2}^{E}((\phi \cos \theta, \phi \sin \theta))= \\
& \underbrace{\left.\frac{2 \sin ^{2} \theta}{\pi \sqrt{\sqrt{E} \phi} \cos \left(2 \pi \sqrt{E} \phi-\frac{\pi}{4}\right.}\right)}_{=: h_{2,2}^{E}(\theta) g_{2,2}^{E}(\phi)}+O\left(\frac{1}{E^{3 / 4} \phi \sqrt{\phi}}\right) \\
& \tilde{r}_{1,2}^{E}((\phi \cos \theta, \phi \sin \theta))=\underbrace{\left.\frac{2 \cos \theta \sin \theta}{\pi \sqrt{\sqrt{E} \phi} \cos \left(2 \pi \sqrt{E} \phi-\frac{\pi}{4}\right.}\right)}_{=: h_{1,2}^{E}(\theta) g_{1,2}^{E}(\phi)}+O\left(\frac{1}{E^{3 / 4} \phi \sqrt{\phi}}\right),
\end{aligned}
$$

uniformly on $(\phi, \theta)$, where the constant involved in the ' $O$ '-notation does not depend on $E$.

Proposition 5.2. Let $_{i, j} \geq 0$ for $i, j=0,1,2$ and $\sum_{i, j=0}^{2} q_{i, j}=4$. Then, as $E \rightarrow+\infty$,

$$
\begin{aligned}
& \int_{\mathcal{D}} \int_{\mathcal{D}} \prod_{i, j=0}^{2} \tilde{r}_{i, j}^{E}(x-y)^{q_{i, j}} d x d y \\
& =\operatorname{area}(\mathcal{D}) \int_{0}^{2 \pi} \prod_{i, j=0}^{2} h_{i, j}^{1}(\theta)^{q_{i, j}} d \theta \cdot \frac{1}{E} \int_{1}^{\sqrt{E} \cdot \operatorname{diam}(\mathcal{D})} \psi \\
& \times \prod_{i, j=0}^{2} g_{i, j}^{1}(\psi)^{q_{i, j}} d \psi+O\left(\frac{1}{E}\right),
\end{aligned}
$$

where functions $h_{i, j}$ and $g_{i, j}$ are defined in (5.70).

Proof. Performing a change of variable for the first term in the r.h.s. of (5.64), we have

$$
\begin{aligned}
& \operatorname{area}(\mathcal{D}) \int_{0}^{\operatorname{diam}(\mathcal{D})} \phi d \phi \int_{0}^{2 \pi} d \theta \prod_{i, j=0}^{2} \widetilde{r}_{i, j}^{E}((\phi \cos \theta, \phi \sin \theta))^{q_{i, j}} \\
& \quad=\frac{\operatorname{area}(\mathcal{D})}{E} \int_{0}^{\sqrt{E} \operatorname{diam}(\mathcal{D})} \psi d \psi \int_{0}^{2 \pi} d \theta \prod_{i, j=0}^{2} \widetilde{r}_{i, j}^{1}((\psi \cos \theta, \psi \sin \theta))^{q_{i, j}}
\end{aligned}
$$




$$
\begin{aligned}
& =\frac{\operatorname{area}(\mathcal{D})}{E} \int_{0}^{1} \psi d \psi \int_{0}^{2 \pi} d \theta \prod_{i, j=0}^{2} \widetilde{r}_{i, j}^{1}((\psi \cos \theta, \psi \sin \theta))^{q_{i, j}} \\
& +\frac{\operatorname{area}(\mathcal{D})}{E} \int_{1}^{\sqrt{E} \operatorname{diam}(\mathcal{D})} \psi d \psi \int_{0}^{2 \pi} d \theta \prod_{i, j=0}^{2} \widetilde{r}_{i, j}^{1}((\psi \cos \theta, \psi \sin \theta))^{q_{i, j}}
\end{aligned}
$$

Since $r^{1}(\psi \cos \theta, \psi \sin \theta) \rightarrow 1, \widetilde{r}_{0, i}^{1}(\psi \cos \theta, \psi \sin \theta)=O(\psi)$ and $\widetilde{r}_{i, i}^{1}(\psi \cos \theta, \psi \sin \theta)$ $\rightarrow 1, \widetilde{r}_{1,2}^{1}(\psi \cos \theta, \psi \sin \theta)=O\left(\psi^{2}\right)$ as $\psi \rightarrow 0$ uniformly on $\theta(i=1,2)$, then from (5.72) we have

$$
\begin{aligned}
& \frac{\operatorname{area}(\mathcal{D})}{E} \int_{0}^{1} \psi d \psi \int_{0}^{2 \pi} d \theta \prod_{i, j=0}^{2} \tilde{r}_{i, j}^{1}((\psi \cos \theta, \psi \sin \theta))^{q_{i, j}} \\
& \quad+\frac{\operatorname{area}(\mathcal{D})}{E} \int_{1}^{\sqrt{E} \operatorname{diam}(\mathcal{D})} \psi d \psi \int_{0}^{2 \pi} d \theta \prod_{i, j=0}^{2} \tilde{r}_{i, j}^{1}((\psi \cos \theta, \psi \sin \theta))^{q_{i, j}} \\
& =O\left(\frac{1}{E}\right)+\frac{\operatorname{area}(\mathcal{D})}{E} \int_{1}^{\sqrt{E} \operatorname{diam}(\mathcal{D})} \psi d \psi \int_{0}^{2 \pi} d \theta \prod_{i, j=0}^{2} \tilde{r}_{i, j}^{1}((\psi \cos \theta, \psi \sin \theta))^{q_{i, j}} .
\end{aligned}
$$

Substituting (5.70) into the last term in the r.h.s. of (5.73) we get

$$
\begin{aligned}
& \frac{\operatorname{area}(\mathcal{D})}{E} \int_{1}^{\sqrt{E} \cdot \operatorname{diam}(\mathcal{D})} \psi d \psi \int_{0}^{2 \pi} d \theta \prod_{i, j=0}^{2} \tilde{r}_{i, j}^{1}((\psi \cos \theta, \psi \sin \theta))^{q_{i, j}} \\
& =\operatorname{area}(\mathcal{D}) \int_{0}^{2 \pi} \prod_{i, j=0}^{2} h_{i, j}^{1}(\theta)^{q_{i, j}} d \theta \cdot \frac{1}{E} \int_{1}^{\sqrt{E} \cdot \operatorname{diam}(\mathcal{D})} \psi \prod_{i, j=0}^{2} g_{i, j}^{1}(\psi)^{q_{i, j}} d \psi \\
& \quad+O\left(\frac{1}{E} \int_{1}^{\sqrt{E} \cdot \operatorname{diam}(\mathcal{D})} \frac{1}{\psi^{2}}\right) \\
& =\operatorname{area}(\mathcal{D}) \int_{0}^{2 \pi} \prod_{i, j=0}^{2} h_{i, j}^{1}(\theta)^{q_{i, j}} d \theta \cdot \frac{1}{E} \int_{1}^{\sqrt{E} \cdot \operatorname{diam}(\mathcal{D})} \\
& \quad \times \prod_{i, j=0}^{2} g_{i, j}^{1}(\psi)^{q_{i, j}} d \psi+O\left(\frac{1}{E}\right) .
\end{aligned}
$$

Substituting (5.74) into (5.73) we prove (5.71).

\section{Fourth Chaotic Components}

6.1. Case of $\mathcal{L}_{E}$. From Equation (3.51) $\mathcal{L}_{E}$ [4], i.e. the projection of $\mathcal{L}_{E}$ onto the fourth chaos, is 


$$
\begin{aligned}
\mathcal{L}_{E}[4]= & \sqrt{2 \pi^{2} E}\left\{\beta_{4} \alpha_{0,0} \int_{\mathcal{D}} H_{4}\left(B_{E}(x)\right) d x\right. \\
& +\beta_{0} \alpha_{4,0} \int_{\mathcal{D}}\left(H_{4}\left(\tilde{\partial_{1}} B_{E}(x)\right)+H_{4}\left(\tilde{\partial_{2}} B_{E}(x)\right)\right) d x \\
& +\beta_{0} \alpha_{2,2} \int_{\mathcal{D}} H_{2}\left(\tilde{\partial_{1}} B_{E}(x)\right) H_{2}\left(\tilde{\partial_{2}} B_{E}(x)\right) d x \\
& \left.+\beta_{2} \alpha_{2,0} \int_{\mathcal{D}} H_{2}\left(B_{E}(x)\right)\left(H_{2}\left(\tilde{\partial_{1}} B_{E}(x)\right)+H_{2}\left(\tilde{\partial_{2}} B_{E}(x)\right)\right) d x\right\} \\
= & \frac{\sqrt{2 \pi^{2} E}}{128}\left\{8 \int_{\mathcal{D}} H_{4}\left(B_{E}(x)\right) d x-\int_{\mathcal{D}}\left(H_{4}\left(\tilde{\partial_{1}} B_{E}(x)\right)+H_{4}\left(\tilde{\partial_{2}} B_{E}(x)\right)\right) d x\right. \\
& -2 \int_{\mathcal{D}} H_{2}\left(\widetilde{\partial_{1}} B_{E}(x)\right) H_{2}\left(\tilde{\partial_{2}} B_{E}(x)\right) d x \\
& \left.-8 \int_{\mathcal{D}} H_{2}\left(B_{E}(x)\right)\left(H_{2}\left(\tilde{\partial_{1}} B_{E}(x)\right)+H_{2}\left(\tilde{\partial_{2}} B_{E}(x)\right)\right) d x\right\} \\
= & \frac{\sqrt{2 \pi^{2} E}}{128}\left\{8 a_{1, E}-a_{2, E}-a_{3, E}-2 a_{4, E}-8 a_{5, E}-8 a_{6, E}\right\},
\end{aligned}
$$

where we used (3.53) and (3.54), and we have set

$$
\begin{aligned}
a_{1, E} & :=\int_{\mathcal{D}} H_{4}\left(B_{E}(x)\right) d x, \quad a_{2, E}:=\int_{\mathcal{D}} H_{4}\left(\widetilde{\partial}_{1} B_{E}(x)\right) d x, \\
a_{3, E} & :=\int_{\mathcal{D}} H_{4}\left(\widetilde{\partial}_{2} B_{E}(x)\right) d x \\
a_{4, E} & :=\int_{\mathcal{D}} H_{2}\left(\widetilde{\partial}_{1} B_{E}(x)\right) H_{2}\left(\widetilde{\partial}_{2} B_{E}(x)\right) d x \\
a_{5, E}: & =\int_{\mathcal{D}} H_{2}\left(B_{E}(x)\right) H_{2}\left(\widetilde{\partial}_{1} B_{E}(x)\right) d x \\
a_{6, E}: & =\int_{\mathcal{D}} H_{2}\left(B_{E}(x)\right) H_{2}\left(\widetilde{\partial}_{2} B_{E}(x)\right) d x .
\end{aligned}
$$

Proposition 6.1. The variance of the fourth chaotic component (6.75) of the nodal length satisfies

$$
\begin{aligned}
\operatorname{Var}\left(\mathcal{L}_{E}[4]\right) & =\frac{\pi^{2} E}{8192} \operatorname{Var}\left(8 a_{1, E}-a_{2, E}-a_{3, E}-2 a_{4, E}-8 a_{5, E}-8 a_{6, E}\right) \\
& \sim \frac{\operatorname{area}(\mathcal{D}) \log E}{512 \pi},
\end{aligned}
$$

where the last asymptotic equivalence holds as $E \rightarrow+\infty$.

In order to prove Proposition 6.1 we need to find the asymptotics, as $E \rightarrow+\infty$, of $\operatorname{Cov}\left(a_{i, E}, a_{j, E}\right)$ for any $i, j \in\{1,2,3,4,5,6\}$ (these results are collected in Lemma 8.5 in Appendix B for simplifying the discussion, and give immediately Proposition 6.1).

Recall first that whenever $U, V, W, Z \sim N(0,1)$ are jointly Gaussian with $\mathbb{E}[U V]=$ $\mathbb{E}[W Z]=0$ : 


$$
\begin{aligned}
\mathbb{E}\left[H_{2}(U) H_{2}(V) H_{2}(W) H_{2}(Z)\right]= & 4 \mathbb{E}[U W]^{2} \mathbb{E}\left[V_{Z}\right]^{2}+4 \mathbb{E}[U Z]^{2} \mathbb{E}[V W]^{2} \\
& +16 \mathbb{E}[U W] \mathbb{E}[U Z] \mathbb{E}[V W] \mathbb{E}[V Z] \\
\mathbb{E}\left[H_{2}(U) H_{2}(V) H_{4}(W)\right]= & 24 \mathbb{E}[U W]^{2} \mathbb{E}[V W]^{2} \\
\mathbb{E}[U V W Z]= & \mathbb{E}[U W] \mathbb{E}[V Z]+\mathbb{E}[U Z] \mathbb{E}[V W]
\end{aligned}
$$

Thanks to (6.77), for any $i, j \in\{1,2,3,4,5,6\}$, $\operatorname{Cov}\left(a_{i, E}, a_{j, E}\right)$ can be written as a finite linear combination of terms of the same form as the 1.h.s. of (5.71).

Recall now that

$$
\begin{aligned}
\cos ^{2} x= & \frac{1}{2}+\frac{1}{2} \cos (2 x), \\
\cos ^{4} x= & \frac{3}{8}+\frac{1}{8} \cos (4 x)+\frac{1}{2} \cos (2 x), \\
\cos ^{6} x= & \frac{5}{16}+\frac{1}{32} \cos (6 x)+\frac{3}{16} \cos (4 x)+\frac{15}{32} \cos (2 x), \\
\cos ^{8} x= & \frac{35}{128}+\frac{1}{128}(56 \cos (2 x)+28 \cos (4 x) \\
& +8 \cos (6 x)+\cos (8 x)) .
\end{aligned}
$$

Taking advantage of (6.78), we can find the asymptotic behavior, as $E \rightarrow+\infty$, of the first term in the r.h.s. of (5.71), thus obtaining the asymptotic behavior of $\operatorname{Cov}\left(a_{i, E}, a_{j, E}\right)$ for any $i, j \in\{1,2,3,4,5,6\}$.

6.2. Case of $\mathcal{N}_{E}$. Using the results of Sect. 3.2 we see that $\mathcal{N}_{E}$ [4], the projection of $\mathcal{N}_{E}$ onto the fourth chaos, is given by

$$
\mathcal{N}_{E}[4]=a_{E}+\widehat{a}_{E}+b_{E},
$$

where

$$
a_{E}=\frac{\pi E}{64}\left\{8 a_{1, E}-a_{2, E}-2 a_{3, E}-8 a_{4, E}\right\}
$$

with $a_{i, E}, i=1, \ldots, 4$ defined in Sect. 6.1, where $\widehat{a}_{E}$ is defined as $a_{E}$ except that we use $\widehat{B}_{E}$ instead of $B_{E}$, and where

$$
\begin{aligned}
b_{E}= & \frac{\pi E}{8}\left\{2 b_{1, E}-b_{2, E}-b_{3, E}-b_{4, E}-b_{5, E}\right. \\
& \left.-\frac{1}{4} b_{6, E}-\frac{1}{4} b_{7, E}+\frac{5}{4} b_{8, E}+\frac{5}{4} b_{9, E}-3 b_{10, E}\right\},
\end{aligned}
$$

with

$$
\begin{aligned}
b_{1, E} & =\int_{\mathcal{D}} H_{2}\left(B_{E}(x)\right) H_{2}\left(\widehat{B}_{E}(x)\right) d x \\
b_{2, E} & =\int_{\mathcal{D}} H_{2}\left(B_{E}(x)\right) H_{2}\left(\widetilde{\partial}_{1} \widehat{B}_{E}(x) d x\right. \\
b_{3, E} & =\int_{\mathcal{D}} H_{2}\left(B_{E}(x)\right) H_{2}\left(\widetilde{\partial}_{2} \widehat{B}_{E}(x)\right) d x
\end{aligned}
$$




$$
\begin{aligned}
b_{4, E} & =\int_{\mathcal{D}} H_{2}\left(\widetilde{\partial}_{1} B_{E}(x)\right) H_{2}\left(\widehat{B}_{E}(x)\right) d x \\
b_{5, E} & =\int_{\mathcal{D}} H_{2}\left(\widetilde{\partial}_{2} B_{E}(x)\right) H_{2}\left(\widehat{B}_{E}(x)\right) d x \\
b_{6, E} & =\int_{\mathcal{D}} H_{2}\left(\widetilde{\partial}_{1} B_{E}(x)\right) H_{2}\left(\widetilde{\partial}_{1} \widehat{B}_{E}(x)\right) d x \\
b_{7, E} & =\int_{\mathcal{D}} H_{2}\left(\widetilde{\partial}_{2} B_{E}(x)\right) H_{2}\left(\widetilde{\partial}_{2} \widehat{B}_{E}(x)\right) d x \\
b_{8, E} & =\int_{\mathcal{D}} H_{2}\left(\widetilde{\partial}_{1} B_{E}(x)\right) H_{2}\left(\widetilde{\partial}_{2} \widehat{B}_{E}(x)\right) d x \\
b_{9, E}= & \int_{\mathcal{D}} H_{2}\left(\widetilde{\partial}_{2} B_{E}(x)\right) H_{2}\left(\widetilde{\partial}_{1} \widehat{B}_{E}(x)\right) d x \\
b_{10, E}= & \int_{\mathcal{D}} \widetilde{\partial}_{1} B_{E}(x) \widetilde{\partial}_{2} B_{E}(x) \widetilde{\partial}_{1} \widehat{B}_{E}(x) \widetilde{\partial}_{2} \widehat{B}_{E}(x) d x .
\end{aligned}
$$

Proposition 6.2. The variance of the fourth chaotic component $\mathcal{N}_{E}$ [4] of $\mathcal{N}_{E}$ is

$$
\operatorname{Var}\left(\mathcal{N}_{E}[4]\right)=2 \operatorname{Var}\left(a_{E}\right)+\operatorname{Var}\left(b_{E}\right) \sim \frac{11 \operatorname{area}(\mathcal{D})}{32 \pi} E \log E,
$$

where the last asymptotics holds as $E \rightarrow+\infty$.

In order to prove Proposition 6.2, observe first from Sect. 6.1 that $a_{E}=\sqrt{2 E} \mathcal{L}_{E}$ [4]. As a result, as $E \rightarrow \infty$, from Proposition 6.1

$$
\operatorname{Var}\left(\widehat{a}_{E}\right)=\operatorname{Var}\left(a_{E}\right) \sim \frac{\operatorname{area}(\mathcal{D}) \log E}{256 \pi} .
$$

So, it remains to consider $b_{E}$. From Lemma 8.11, which is collected in Appendix B to simplify the discussion, we have the following.

\section{Lemma 6.3.}

$$
\begin{aligned}
\operatorname{Var}\left(b_{E}\right)= & \frac{\pi^{2} E^{2}}{64} \operatorname{Var}\left(2 b_{1, E}-b_{2, E}-b_{3, E}-b_{4, E}-b_{5, E}-\frac{1}{4} b_{6, E}-\frac{1}{4} b_{7, E}\right. \\
& \left.+\frac{5}{4} b_{8, E}+\frac{5}{4} b_{9, E}-3 b_{10, E}\right) \sim \frac{43 \operatorname{area}(\mathcal{D})}{128 \pi} E \log E
\end{aligned}
$$

where the last asymptotics holds as $E \rightarrow+\infty$.

Proof of Proposition 6.2. From (6.81), observing that $a_{E}, \widehat{a}_{E}$ and $b_{E}$ are indeed uncorrelated from Lemma 8.11 and Lemma 6.3, we obtain (6.80).

\section{Higher Order Chaotic Components}

7.1. Preliminaries. Let us start with the following result, whose proof is elementary (see Lemma 3.1) and hence omitted. 
Lemma 7.1. The map (see (1.9))

$$
\mathbb{R}^{2} \ni x \mapsto r^{E}(x / \sqrt{E})
$$

and its derivatives up to the order two are Lipschitz with a universal Lipschitz constant $c>0$, in particular independent of $E$.

Let us now consider a square $Q$ of side length $d=\operatorname{diam}(\mathcal{D})$ which contains $\mathcal{D}$, and $M:=\lceil\gamma \sqrt{E}\rceil$, where $\gamma$ will be chosen in a while. Let $\left\{Q_{i}: 1, \ldots, M^{2}\right\}$ be a partition of $Q$ in $M^{2}$ squares of side length $d / M$. Let $0<\varepsilon<1 / 1000$ be a fixed small number, and now choose $\gamma \geq \frac{4 \sqrt{2} c \cdot d}{\varepsilon}$, where $c$ is the Lipschitz constant in Lemma 7.1. The following is inspired by [ORW08, RW16].

Definition 7.2. The pair $\left(Q_{i}, Q_{j}\right)$ is singular if there exists $(x, y) \in Q_{i} \times Q_{j}$, as well as $k, l \in\{0,1,2\}$, such that (see (5.62))

$$
\left|\widetilde{r}_{k, l}^{E}(x-y)\right| \geq \varepsilon .
$$

Lemma 7.3. If $\left(Q_{i}, Q_{j}\right)$ is singular, then $\exists k, l \in\{0,1,2\}$ such that $\forall(x, y) \in Q_{i} \times Q_{j}$ we have

$$
\left|\tilde{r}_{k, l}^{E}(x-y)\right| \geq \frac{\varepsilon}{2}
$$

Proof. Assume that $(x, y) \in Q_{i} \times Q_{j}$ is such that $r^{E}(x-y) \geq \varepsilon$. For $(z, w) \in Q_{i} \times Q_{j}$ we have, from Lemma 7.1,

$$
\begin{aligned}
\left|r^{E}(z-w)-r^{E}(x-y)\right| & =\left|r^{E}\left(\frac{(z-w) \sqrt{E}}{\sqrt{E}}\right)-r^{E}\left(\frac{(x-y) \sqrt{E}}{\sqrt{E}}\right)\right| \\
& \leq c \cdot \sqrt{E}|(z-x)-(w-y)| \leq 2 c \cdot \sqrt{E} \cdot \frac{\sqrt{2} \cdot d}{M} .
\end{aligned}
$$

It hence follows that (recall the definition of $M$ )

$$
r^{E}(z-w) \geq r(x-y)-2 c \cdot \sqrt{E} \cdot \frac{\sqrt{2} \cdot d}{M} \geq \frac{\varepsilon}{2} .
$$

The proof for $r^{E}(x-y) \leq-\varepsilon$ is similar, as well as that one in the case of singularities w.r.t. derivatives.

For each $Q_{i}$ consider $\mathcal{D} \cap Q_{i}$ and, if it is not empty, set $\mathcal{D}_{i}:=\mathcal{D} \cap Q_{i}$. The set $\left\{\mathcal{D}_{i}\right\}$ is hence a partition of $\mathcal{D}$. Let $\mathcal{D}_{1}, Q_{1}$ be the sets containing the origin (note that for sufficiently large $E, \mathcal{D}_{1}$ and $Q_{1}$ coincide). In view of Lemma 7.3 we give the following.

Definition 7.4. We say that $\left(\mathcal{D}_{i}, \mathcal{D}_{j}\right)$ is singular if $\left(Q_{i}, Q_{j}\right)$ is singular.

The proof of the following result is analogous to the proof of Lemma 6.3 in [DNPR16], and hence omitted. 
Lemma 7.5. For a fixed cell $\mathcal{D}_{i}$, the number $N_{i}$ of $j \in\left\{1,2, \ldots, M^{2}\right\}$ such that $\left(\mathcal{D}_{i}, \mathcal{D}_{j}\right)$ is singular is

$$
N_{i}=O\left(E \cdot \max _{k, l \in\{0,1,2\}} \int_{\mathcal{D}} \int_{\mathcal{D}} \widetilde{r}_{k, l}^{E}(x-y)^{6} d x d y\right),
$$

where the constant involved in the ' $O^{\prime}$-notation depend nor on E neither on $i$.

The following lemma will be proven in Appendix C.

Lemma 7.6. $\forall k, l \in\{0,1,2\}$, as $E \rightarrow+\infty$,

$$
\int_{\mathcal{D}} \int_{\mathcal{D}} \widetilde{r}_{k, l}^{E}(x-y)^{6} d x d y=o\left(\frac{\log E}{E}\right) .
$$

Lemma 7.7. Let $\mathcal{L}_{E}\left(\mathcal{D}_{1}\right)$ denote the nodal length of $B_{E}$ inside $\mathcal{D}_{1}$. Then

$$
\mathbb{E}\left[\mathcal{L}_{E}\left(\mathcal{D}_{1}\right)^{2}\right]=O\left(\frac{1}{E}\right)
$$

Proof. It follows from the proof of Lemma 3.3 that

$$
\begin{aligned}
& \mathbb{E}\left[\mathcal{L}_{E}\left(\mathcal{D}_{1}\right)^{2}\right] \\
& =\int_{\mathcal{D}_{1}} \int_{\mathcal{D}_{1}} \mathbb{E}\left[\left\|\nabla B_{E}(x)\right\|\left\|\nabla B_{E}(y)\right\| \mid B_{E}(x)=B_{E}(y)=0\right] p_{\left(B_{E}(x), B_{E}(y)\right)}(0,0) d x d y \\
& \ll \int_{\mathcal{D}_{1}} \int_{\mathcal{D}_{1}} \frac{E}{\sqrt{E}\|x-y\|} d x d y=O\left(\frac{1}{E}\right) .
\end{aligned}
$$

7.2. Residual terms. For a random variable $F$ in $L^{2}(\mathbb{P})$, let us denote by $F \mid C_{\geq 6}$ the projection of $F$ onto $C_{\geq 6}:=\bigoplus_{q=6}^{+\infty} C_{q}$.

Let us start investigating the case of the nodal length. We can write

$$
\begin{aligned}
\operatorname{Var}\left(\mathcal{L}_{E} \mid C_{\geq 6}\right)= & \sum_{\left(\mathcal{D}_{i}, \mathcal{D}_{j}\right) \text { sing. }} \operatorname{Cov}\left(\operatorname{proj}\left(\mathcal{L}_{E}\left(\mathcal{D}_{i}\right) \mid C_{\geq 6}\right), \operatorname{proj}\left(\mathcal{L}_{E}\left(\mathcal{D}_{j}\right) \mid C_{\geq 6}\right)\right) \\
+ & \sum_{\left(\mathcal{D}_{i}, \mathcal{D}_{j}\right) \text { non-sing. }} \operatorname{Cov}\left(\operatorname{proj}\left(\mathcal{L}_{E}\left(\mathcal{D}_{i}\right) \mid C_{\geq 6}\right), \operatorname{proj}\left(\mathcal{L}_{E}\left(\mathcal{D}_{j}\right) \mid C_{\geq 6}\right)\right) \\
= & : X(E)+Y(E) .
\end{aligned}
$$

We are going to separately investigate the two terms $X(E)$ and $Y(E)$.

Lemma 7.8. The contribution of non-singular pairs of cells is, as $E \rightarrow+\infty$,

$$
Y(E)=o(\log E) .
$$


Proof. Reasoning as in the second part of the proof of Lemma 2 in [PR17], we find

$$
\begin{gathered}
|Y(E)| \leq 2 \pi^{2} E \sum_{q \geq 3} \sum_{i_{1}+i_{2}+i_{3}=q} \sum_{j_{1}+j_{2}+j_{3}=q}\left|\beta_{2 i_{1}} \alpha_{2 i_{2}, 2 i_{3}}\right|\left|\beta_{2 j_{1}} \alpha_{2 j_{2}, 2 j_{3}}\right| \\
\times 1_{i_{1}+i_{2}+i_{3}=j_{1}+j_{2}+j_{3}}\left|U\left(i_{1}, i_{2}, i_{3}, j_{1}, j_{2}, j_{3}\right)\right|,
\end{gathered}
$$

where $U\left(i_{1}, i_{2}, i_{3}, j_{1}, j_{2}, j_{3}\right)$ (for $i_{1}+i_{2}+i_{3}=q$ ) is a sum of at most (2q)! terms of the form

$$
\sum_{\left(\mathcal{D}_{i}, \mathcal{D}_{j}\right) \text { non-sing. }} \int_{\mathcal{D}_{i}} \int_{\mathcal{D}_{j}} \prod_{u=1}^{2 q} \tilde{r}_{l_{u}, k_{u}}^{E}(x-y) d x d y
$$

where $l_{u}, k_{u} \in\{0,1,2\}$. Since $2 q \geq 6$, and we are working on non-singular pairs of cells (see Definition 7.2), from (7.83) we can write

$$
\begin{aligned}
& \left|\sum_{\left(\mathcal{D}_{i}, \mathcal{D}_{j}\right) \text { non-sing. }} \int_{\mathcal{D}_{i}} \int_{\mathcal{D}_{j}} \prod_{u=1}^{2 q} \widetilde{r}_{l_{u}, k_{u}}^{E}(x-y) d x d y\right| \\
& \leq \varepsilon^{2 q-6} \int_{\mathcal{D}} \int_{\mathcal{D}} \prod_{u=1}^{6}\left|\widetilde{r}_{l_{u}, k_{u}}^{E}(x-y)\right| d x d y .
\end{aligned}
$$

Substituting (7.84) into (7.82) we get

$$
\begin{aligned}
|Y(E)| \leq & 2 \pi^{2} E \sum_{q \geq 3}(2 q) ! \sum_{i_{1}+i_{2}+i_{3}=q} \sum_{j_{1}+j_{2}+j_{3}=q}\left|\beta_{2 i_{1}} \alpha_{2 i_{2}, 2 i_{3}}\right|\left|\beta_{2 j_{1}} \alpha_{2 j_{2}, 2 j_{3}}\right| \\
& \times \varepsilon^{i_{1}+i_{2}+i_{3}} \varepsilon^{j_{1}+j_{2}+j_{3}} \frac{\int_{\mathcal{D}} \int_{\mathcal{D}} \prod_{u=1}^{6}\left|\widetilde{r}_{l_{u}, k_{u}}^{E}(x-y)\right| d x d y}{\varepsilon^{6}} \\
\leq & 2 \pi^{2} E \sum_{q \geq 3}(2 q) ! \sum_{i_{1}+i_{2}+i_{3}=q} \sum_{j_{1}+j_{2}+j_{3}=q}\left|\beta_{2 i_{1}} \alpha_{2 i_{2}, 2 i_{3}}\right|\left|\beta_{2 j_{1}} \alpha_{2 j_{2}, 2 j_{3}}\right| \\
& \times \varepsilon^{i_{1}+i_{2}+i_{3}} \varepsilon^{j_{1}+j_{2}+j_{3}} \frac{\max _{l_{u}, k_{u} \in\{0,1,2\}} \int_{\mathcal{D}} \int_{\mathcal{D}} \prod_{u=1}^{6}\left|\widetilde{r}_{l_{u}, k_{u}}^{E}(x-y)\right| d x d y}{\varepsilon^{6}} \\
= & 2 \pi^{2} E \cdot S \cdot \frac{\max _{l_{u}, k_{u} \in\{0,1,2\}} \int_{\mathcal{D}} \int_{\mathcal{D}} \prod_{u=1}^{6}\left|\widetilde{r}_{l_{u}, k_{u}}^{E}(x-y)\right| d x d y}{\varepsilon^{6}}
\end{aligned}
$$

where

$$
S:=\sum_{q \geq 3}(2 q) ! \sum_{i_{1}+i_{2}+i_{3}=q} \sum_{j_{1}+j_{2}+j_{3}=q}\left|\beta_{2 i_{1}} \alpha_{2 i_{2}, 2 i_{3}}\right|\left|\beta_{2 j_{1}} \alpha_{2 j_{2}, 2 j_{3}}\right| \varepsilon^{i_{1}+i_{2}+i_{3}} \varepsilon^{j_{1}+j_{2}+j_{3}} .
$$

As $E \rightarrow+\infty$,

$$
\frac{\max _{l_{u}, k_{u} \in\{0,1,2\}} \int_{\mathcal{D}} \int_{\mathcal{D}} \prod_{u=1}^{6}\left|\widetilde{r}_{l_{u}, k_{u}}^{E}(x-y)\right| d x d y}{\varepsilon^{6}}=o\left(\frac{\log E}{E}\right) .
$$


(The proof of (7.86) is analogous to the proof in Appendix C of Lemma 7.6 and hence omitted.) Reasoning as in the proof of [DNPR16, Lemma 3.5] (or the proof of [PR17, Lemma 2]) we have

$$
\begin{aligned}
S & \leq \sum_{q \geq 0}(2 q) ! \sum_{i_{1}+i_{2}+i_{3}=q} \sum_{j_{1}+j_{2}+j_{3}=q}\left|\beta_{2 i_{1}} \alpha_{2 i_{2}, 2 i_{3}}\right|\left|\beta_{2 j_{1}} \alpha_{2 j_{2}, 2 j_{3}}\right| \varepsilon^{i_{1}+i_{2}+i_{3}} \varepsilon^{j_{1}+j_{2}+j_{3}} \\
& \leq \sum_{i_{1}, i_{2}, i_{3}}\left(2 i_{1}+2 i_{2}+2 i_{3}\right) !\left|\beta_{2 i_{1}} \alpha_{2 i_{2}, 2 i_{3}}\right|^{2} \varepsilon^{i_{1}+i_{2}+i_{3}}<+\infty
\end{aligned}
$$

Substituting (7.87) and (7.86) into (7.85) we conclude the proof.

Lemma 7.9. The contribution of singular pairs of cells is, as $E \rightarrow+\infty$,

$$
X(E)=o(\log E) .
$$

Proof. Reasoning as in the first part of the proof of Lemma 2 in [PR17]

$$
X(E) \ll E \cdot N_{1} \cdot \mathbb{E}\left[\mathcal{L}_{E}\left(\mathcal{D}_{1}\right)^{2}\right] \ll E \cdot \max _{k, l \in\{0,1,2\}} \int_{\mathcal{D}} \int_{\mathcal{D}} \widetilde{r}_{k, l}^{E}(x-y)^{6} d x d y=o(\log E),
$$

where for the last step we used Lemma 7.5, (7.86) and Lemma 7.7.

Let us now investigate the case of nodal points.

Lemma 7.10. As $E \rightarrow+\infty$,

$$
\operatorname{Var}\left(\mathcal{N}_{E} \mid C_{\geq 6}\right)=o(E \log E) .
$$

Proof. Let us first write

$$
\begin{aligned}
\operatorname{Var}\left(\mathcal{N}_{E} \mid C_{\geq 6}\right)= & \sum_{\left(\mathcal{D}_{i}, \mathcal{D}_{j}\right) \text { sing. }} \operatorname{Cov}\left(\operatorname{proj}\left(\mathcal{N}_{E}\left(\mathcal{D}_{i}\right) \mid C_{\geq 6}\right), \operatorname{proj}\left(\mathcal{N}_{E}\left(\mathcal{D}_{j}\right) \mid C_{\geq 6}\right)\right) \\
+ & \sum_{\left(\mathcal{D}_{i}, \mathcal{D}_{j}\right) \text { non-sing. }} \operatorname{Cov}\left(\operatorname{proj}\left(\mathcal{N}_{E}\left(\mathcal{D}_{i}\right) \mid C_{\geq 6}\right), \operatorname{proj}\left(\mathcal{N}_{E}\left(\mathcal{D}_{j}\right) \mid C_{\geq 6}\right)\right) \\
= & : X(E)+Y(E) .
\end{aligned}
$$

The contribution of the singular part corresponding to the term $X(E)$ can be investigated as in the proof of Lemma 3.4 in [DNPR16]:

$$
\begin{aligned}
& \left|\sum_{\left(\mathcal{D}_{i}, \mathcal{D}_{j}\right) \text { sing. }} \operatorname{Cov}\left(\operatorname{proj}\left(\mathcal{N}_{E}\left(\mathcal{D}_{i}\right) \mid C_{\geq 6}\right), \operatorname{proj}\left(\mathcal{N}_{E}\left(\mathcal{D}_{j}\right) \mid C_{\geq 6}\right)\right)\right| \\
& \ll E \cdot N_{1} \cdot\left(\mathbb{E}\left[\mathcal{N}_{E}\left(\mathcal{D}_{1}\right)\left(\mathcal{N}_{E}\left(\mathcal{D}_{1}\right)-1\right)\right]+\mathbb{E}\left[\mathcal{N}_{E}\left(\mathcal{D}_{1}\right)\right]\right),
\end{aligned}
$$

where $N_{1}$ is defined in Lemma 7.5, and $\mathcal{D}_{1}$ still denotes the cell around the origin. From Theorem 1.4, in particular (1.15), we have $\mathbb{E}\left[\mathcal{N}_{E}\left(\mathcal{D}_{1}\right)\right]=O(1)$, while from standard Kac-Rice formula for the second factorial moment of $\mathcal{N}_{E}$ restricted to $\mathcal{D}_{1}$ and Lemma 8.20 it follows that

$$
\mathbb{E}\left[\mathcal{N}_{E}\left(\mathcal{D}_{1}\right)\left(\mathcal{N}_{E}\left(\mathcal{D}_{1}\right)-1\right)\right]=O(1)
$$


Substituting (7.90) into (7.89), from Lemma 7.5 we obtain, as $E \rightarrow+\infty$,

$$
X(E)=o(E \log E) .
$$

The remaining term $Y(E)$ which corresponds to the non-singular part can be investigated as in the proof of Lemma 3.5 in [DNPR16] and the proof of Lemma 7.8 (the details ore omitted for the sake of brevity): as $E \rightarrow+\infty$,

$$
Y(E)=o(E \log E) .
$$

Substituting (7.92) and (7.91) into (7.88) we can conclude the proof.

\section{Proofs of the Main Results}

8.1. Central limit theorems. In this section we implicitly represent $B_{E}$ and its first derivatives in terms of a real Gaussian measure (cf. (3.43)), allowed by isometric property between Hilbert spaces. We prove asymptotic Gaussianity, as $E \rightarrow+\infty$, for fourth order components $\mathcal{L}_{E}$ [4] and $\mathcal{N}_{E}$ [4] in (6.75) and (6.79), respectively. According to [PT05] and because we already checked the convergence of covariances (of summands in both (6.75) and (6.79)) in Sect. 6 (and in Lemmas 8.5-8.19), it suffices to prove that each of those summands satisfies a CLT. To this aim, we apply Fourth Moment Theorem [NP12, NuPe05]; this technique requires to control the asymptotic behavior of nontrivial contraction norms (see [NP12, §B.4]) of each term mentioned above. The latter goal is achieved by using the key result contained in the following statement (see the proof of Proposition 8.2).

Lemma 8.1. Fix integers $1 \leq a_{1}, \ldots, a_{4} \leq 2$ and $1 \leq b_{1}, \ldots, b_{4} \leq 3$ such that $b_{1}+\ldots+b_{4}=8$. Then the quantity

$$
\begin{aligned}
& \frac{E^{2}}{\log ^{2} E} \int_{\mathcal{D}^{4}}\left|J_{a_{1}}\left(2 \pi \sqrt{E}\left\|x_{1}-x_{2}\right\|\right)\right|^{b_{1}}\left|J_{a_{2}}\left(2 \pi \sqrt{E}\left\|x_{2}-x_{3}\right\|\right)\right|^{b_{2}} \\
& \times\left|J_{a_{3}}\left(2 \pi \sqrt{E}\left\|x_{3}-x_{4}\right\|\right)\right|^{b_{3}}\left|J_{a_{4}}\left(2 \pi \sqrt{E}\left\|x_{4}-x_{1}\right\|\right)\right|^{b_{4}} d x_{1} \ldots d x_{4} \\
& =: u_{E}
\end{aligned}
$$

goes to zero, as $E \rightarrow \infty$.

Proof. Performing a change of variables we can write

$$
\begin{aligned}
u_{E}= & \frac{1}{E^{2} \log ^{2} E} \int_{(\sqrt{E} \mathcal{D})^{4}}\left|J_{a_{1}}\left(2 \pi\left\|x_{1}-x_{2}\right\|\right)\right|^{b_{1}}\left|J_{a_{2}}\left(2 \pi\left\|x_{2}-x_{3}\right\|\right)\right|^{b_{2}} \\
& \times\left|J_{a_{3}}\left(2 \pi\left\|x_{3}-x_{4}\right\|\right)\right|^{b_{3}}\left|J_{a_{4}}\left(2 \pi\left\|x_{4}-x_{1}\right\|\right)\right|^{b_{4}} d x_{1} \ldots d x_{4} .
\end{aligned}
$$

If, for all $i>j$ we had that $b_{i}+b_{j}>4$, then we would have $3\left(b_{1}+\ldots+b_{4}\right)>24$, which contradicts that $b_{1}+\ldots+b_{4}=8$. By symmetry, we can thus assume without loss of generality that $b_{1}+b_{2} \leq 4$ and then use that $x^{b_{1}} y^{b_{2}} \leq x^{b_{1}+b_{2}}+y^{b_{1}+b_{2}}$. This way, we get that $u_{E}$ is less than

$$
\begin{aligned}
\frac{1}{E^{2} \log ^{2} E} \int_{(\sqrt{E} \mathcal{D})^{4}} & \left|J_{a_{1}}\left(2 \pi\left\|x_{1}-x_{2}\right\|\right)\right|^{b_{1}+b_{2}}\left|J_{a_{3}}\left(2 \pi\left\|x_{3}-x_{4}\right\|\right)\right|^{b_{3}} \\
& \times\left|J_{a_{4}}\left(2 \pi\left\|x_{4}-x_{1}\right\|\right)\right|^{b_{4}} d x_{1} \ldots d x_{4}
\end{aligned}
$$


plus a similar term. Now, let us apply the change of variables $u=x_{1}-x_{2}, v=x_{3}-x_{4}$, $w=x_{4}-x_{1}$ and $z=x_{1}$ in (8.93). We obtain that (8.93) is less or equal than

$$
\begin{aligned}
& \frac{\operatorname{Area}(\mathcal{D})}{E \log ^{2} E} \int_{\sqrt{E}(\mathcal{D}-\mathcal{D})}\left|J_{a_{1}}(2 \pi\|u\|)\right|^{b_{1}+b_{2}} d u \\
& \quad \times \int_{\sqrt{E}(\mathcal{D}-\mathcal{D})}\left|J_{a_{3}}(2 \pi\|v\|)\right|^{b_{3}} d v \int_{\sqrt{E}(\mathcal{D}-\mathcal{D})}\left|J_{a_{4}}(2 \pi\|w\|)\right|^{b_{4}} d w .
\end{aligned}
$$

But $\left|J_{a}(2 \pi r)\right| \leq \operatorname{cst}(a) r^{-\frac{1}{2}}$ for any $r>0$ and $a \in\{0,1,2\}$ so that, for any $b \in$ $\{1,2,3,4\}$,

$$
\begin{aligned}
& \int_{\sqrt{E}(\mathcal{D}-\mathcal{D})}\left|J_{a}(2 \pi\|u\|)\right|^{b} d u \leq \operatorname{cst}(a, b) \int^{\sqrt{E}} r^{1-\frac{b}{2}} d r \\
& \leq \operatorname{cst}(a, b)\left\{\begin{array}{ll}
E^{1-\frac{b}{4}} & \text { if } b=1,2,3 \\
\log E & \text { if } b=4
\end{array} .\right.
\end{aligned}
$$

Substituting (8.95) in (8.94) and recalling that $1 \leq b_{1}+b_{2} \leq 4,1 \leq b_{3}, b_{4} \leq 3$ and $b_{1}+\ldots+b_{4}=8$, we obtain that (8.94) is less or equal than

$$
\frac{\operatorname{area}(\mathcal{D})}{E \log ^{2} E} \times E^{1-\frac{b_{1}+b_{2}}{4}} \log E \times E^{1-\frac{b_{3}}{4}} \times E^{1-\frac{b_{4}}{4}}=O\left((\log E)^{-1}\right) \rightarrow 0, \quad \text { as } E \rightarrow \infty
$$

We can now prove the main result of this subsection.

Proposition 8.2. As $E \rightarrow+\infty$,

$$
\frac{\mathcal{L}_{E}[4]}{\sqrt{\operatorname{Var}\left(\mathcal{L}_{E}[4]\right)}} \stackrel{d}{\rightarrow} Z
$$

and

$$
\frac{\mathcal{N}_{E}[4]}{\sqrt{\operatorname{Var}\left(\mathcal{N}_{E}[4]\right)}} \stackrel{d}{\rightarrow} Z
$$

where $Z$ is a standard Gaussian random variable.

Remark 8.3. The proof of Proposition 8.2 provided below follows a standard strategy, that can be roughly described as follows (see [NP12, Chapter 5] for an exhaustive discussion):

1. for every $E>0$, represent the random variable

$$
U(E)=\frac{\mathcal{L}_{E}[4]}{\sqrt{\operatorname{Var}\left(\mathcal{L}_{E}[4]\right)}} \text { or } \frac{\mathcal{N}_{E}[4]}{\sqrt{\operatorname{Var}\left(\mathcal{N}_{E}[4]\right)}}
$$

as a linear combination of unit variance multiple integral of order 4 , that is, random variables with the form

$$
I_{4}\left(f_{E}\right)=\int_{A} \cdots \int_{A} f_{E}\left(x_{1}, \ldots, x_{4}\right) G\left(d x_{1}\right) \cdots G\left(d_{4}\right),
$$

where $G$ is a Gaussian measure over some measurable space $(A, \mathscr{A})$, controlled by a $\sigma$-finite deterministic positive measure $\mu$ (see [NP12, p. 24]), and $f_{E}$ is a suitable symmetric kernel; 
2. apply [NP12, Theorem 5.2.7] to deduce the asymptotic normality of each $I_{4}\left(f_{E}\right)$ by showing that the two non-trivial contractions (see [NP12, §B.4]) $f_{E} \otimes_{1} f_{E}$ and $f_{E} \otimes_{2} f_{E}$ converge to zero in square norm;

3. recall the definitions

$$
\begin{aligned}
f_{E} \otimes_{1} f_{E}\left(x_{1}, x_{2}, x_{3}, y_{1}, y_{2}, y_{3}\right) & :=\int_{A} f_{E}\left(x_{1}, x_{2}, x_{3}, z\right) f_{E}\left(y_{1}, y_{2}, y_{3}, z\right) \mu(d z), \\
f_{E} \otimes_{2} f_{E}\left(x_{1}, x_{2}, y_{1}, y_{2},\right) & :=\int_{A} \int_{A} f_{E}\left(x_{1}, x_{2}, v, z\right) f_{E}\left(y_{1}, y_{2}, v, z\right) \mu(d v) \mu(d z) ;
\end{aligned}
$$

4. use the main result in [PT05] to deduce the asymptotic normality of $U(E)$ by using the fact that all covariances between the multiple integrals at the previous item converge to appropriate limits, as $E \rightarrow \infty$ (this corresponds to the content of Sect. 6 above).

Proof of Proposition 8.2. Recall the expressions for fourth order chaotic components in (6.75) and (6.79). According to [PT05] and because we already checked the convergence of covariances in Sect. 6, it remains to check that the two non-trivial contractions (see the discussion above) associated with the fourth order Wiener-Itô integrals $\sqrt{\frac{E}{\log E}} a_{i, E}$ $(1 \leq i \leq 6)$ and $\sqrt{\frac{E}{\log E}} b_{j, E}(1 \leq j \leq 10)$ in (6.75) and (6.79) go to zero as $E \rightarrow \infty$. Due to the high number of terms that are involved, we only show how to check this on a particular term that is representative of the difficulty. All the other calculations follow exactly the same line, relying on Lemma 8.1. Let us consider

$$
\sqrt{\frac{E}{\log E}} b_{2, E}=I_{4}\left(\widetilde{\alpha_{E}}\right)
$$

with

$$
\alpha_{E}\left(u_{1}, \ldots, u_{4}\right):=\sqrt{\frac{E}{\log E}} \int_{\mathcal{D}} f_{E}\left(x, u_{1}\right) f_{E}\left(x, u_{2}\right) g_{E}\left(x, u_{3}\right) g_{E}\left(x, u_{4}\right) d x .
$$

Here $f_{E}(x, \cdot)$ and $g_{E}(x, \cdot)$ are chosen so that $B_{E}(x)=I_{1}\left(f_{E}(x, \cdot)\right)$ and $\widetilde{\partial}_{1} \widehat{B}_{E}(x)=$ $I_{1}\left(g_{E}(x, \cdot)\right)$ respectively, where $I_{k}$ indicates a multiple integral of order $k$ with respect to an appropriate real-valued Gaussian measure-see Remark 3.4. The symmetrization $\widetilde{\alpha_{E}}$ of $\alpha_{E}$ is given by

$$
\begin{aligned}
\widetilde{\alpha_{E}}\left(u_{1}, \ldots, u_{4}\right):= & \frac{1}{6} \sqrt{\frac{E}{\log E}} \int_{\mathcal{D}}\left\{f_{E}\left(x, u_{1}\right) f_{E}\left(x, u_{2}\right) g_{E}\left(x, u_{3}\right) g_{E}\left(x, u_{4}\right)\right. \\
& +f_{E}\left(x, u_{1}\right) g_{E}\left(x, u_{2}\right) f_{E}\left(x, u_{3}\right) g_{E}\left(x, u_{4}\right) \\
& +f_{E}\left(x, u_{1}\right) g_{E}\left(x, u_{2}\right) g_{E}\left(x, u_{3}\right) f_{E}\left(x, u_{4}\right) \\
& +g_{E}\left(x, u_{1}\right) f_{E}\left(x, u_{2}\right) f_{E}\left(x, u_{3}\right) g_{E}\left(x, u_{4}\right) \\
& +g_{E}\left(x, u_{1}\right) f_{E}\left(x, u_{2}\right) g_{E}\left(x, u_{3}\right) f_{E}\left(x, u_{4}\right) \\
& \left.+g_{E}\left(x, u_{1}\right) g_{E}\left(x, u_{2}\right) f_{E}\left(x, u_{3}\right) f_{E}\left(x, u_{4}\right)\right\} d x .
\end{aligned}
$$

Let us now consider, for instance, the first contraction $\widetilde{\alpha_{E}} \otimes_{1} \widetilde{\alpha_{E}}$. It is given by a sum of 36 terms. They are all of the same order. For instance, it contains the term 


$$
\begin{aligned}
& \left(u_{1}, u_{2}, u_{3}, v_{1}, v_{2}, v_{3}\right) \mapsto \frac{E}{36 \log E} \int_{\mathcal{D}^{2}} f_{E}\left(x_{1}, u_{1}\right) f_{E}\left(x_{1}, u_{2}\right) \\
& \quad \times g_{E}\left(x_{1}, u_{3}\right) f_{E}\left(x_{2}, v_{1}\right) g_{E}\left(x_{2}, v_{2}\right) g_{E}\left(x_{2}, v_{3}\right) \mathbb{E}\left[\widetilde{\partial}_{1} \widehat{B}_{E}\left(x_{1}\right) B_{E}\left(x_{2}\right)\right] d x_{1} d x_{2} .
\end{aligned}
$$

Then, $\left\|\widetilde{\alpha_{E}} \otimes_{1} \widetilde{\alpha_{E}}\right\|^{2}$ is given by a sum of $36^{2}$ terms, which all behave the same way. One of them (corresponding to (8.96) above) is given by

$$
\begin{aligned}
& \frac{E^{2}}{36^{2} \log ^{2} E} \int_{\mathcal{D}^{4}} \mathbb{E}\left[B_{E}\left(x_{1}\right) B_{E}\left(x_{3}\right)\right]^{2} \mathbb{E}\left[\widetilde{\partial}_{1} \widehat{B}_{E}\left(x_{1}\right) \widetilde{\partial}_{1} \widehat{B}_{E}\left(x_{3}\right)\right] \mathbb{E}\left[B_{E}\left(x_{2}\right) B_{E}\left(x_{4}\right)\right] \\
& \quad \times \mathbb{E}\left[\widetilde{\partial}_{1} \widehat{B}_{E}\left(x_{2}\right) \widetilde{\partial}_{1} \widehat{B}_{E}\left(x_{4}\right)\right]^{2} \mathbb{E}\left[\widetilde{\partial}_{1} \widehat{B}_{E}\left(x_{1}\right) B_{E}\left(x_{2}\right)\right] \\
& \quad \times \mathbb{E}\left[\widetilde{\partial}_{1} \widehat{B}_{E}\left(x_{3}\right) B_{E}\left(x_{4}\right)\right] d x_{1} \ldots d x_{4} .
\end{aligned}
$$

Using Lemma 3.1, we obtain that the absolute value of (8.97) is less or equal than (up to universal constants whose exact value are immaterial)

$$
\begin{aligned}
& \frac{E^{2}}{\log ^{2} E} \int_{\mathcal{D}^{4}}\left(\left|J_{0}\left(2 \pi \sqrt{E}\left\|x_{1}-x_{3}\right\|\right)\right|^{3}+\left|J_{2}\left(2 \pi \sqrt{E}\left\|x_{1}-x_{3}\right\|\right)\right|^{3}\right) \\
& \quad \times\left(\left|J_{0}\left(2 \pi \sqrt{E}\left\|x_{2}-x_{4}\right\|\right)\right|^{3}+\left|J_{2}\left(2 \pi \sqrt{E}\left\|x_{2}-x_{4}\right\|\right)\right|^{3}\right) \\
& \quad \times\left|J_{1}\left(2 \pi \sqrt{E}\left\|x_{1}-x_{2}\right\|\right)\right| \times\left|J_{1}\left(2 \pi \sqrt{E}\left\|x_{3}-x_{4}\right\|\right)\right| d x_{1} \ldots d x_{4}
\end{aligned}
$$

and thus goes to zero as $E \rightarrow \infty$ thanks to Lemma 8.1.

8.2. Proofs of Theorems 1.1 and 1.4. In this subsection we prove our main results.

Proof of Theorem 1.1. Consider the chaotic expansion for the nodal length $\mathcal{L}_{E}$ in (3.51). For the 0-th chaotic component we have

$$
\mathcal{L}_{E}[0]=\mathbb{E}\left[\mathcal{L}_{E}\right]=\operatorname{area}(\mathcal{D}) \sqrt{2 \pi^{2} E} \beta_{0} \alpha_{0,0}=\operatorname{area}(\mathcal{D}) \frac{\pi}{\sqrt{2}} \sqrt{E},
$$

where we used (3.53) and (3.54). By (4.57), (6.76) and Lemmas 7.9, 7.8 we deduce that, as $E \rightarrow+\infty$,

$$
\operatorname{Var}\left(\mathcal{L}_{E}\right) \sim \operatorname{Var}\left(\mathcal{L}_{E}[4]\right)
$$

and

$$
\frac{\mathcal{L}_{E}-\mathbb{E}\left[\mathcal{L}_{E}\right]}{\sqrt{\operatorname{Var}\left(\mathcal{L}_{E}\right.}}=\frac{\mathcal{L}_{E}[4]}{\sqrt{\operatorname{Var}\left(\mathcal{L}_{E}[4]\right.}}+o_{\mathbb{P}}(1),
$$

where $o_{\mathbb{P}}(1)$ denotes a sequence converging to zero in probability. Proposition 8.2 allows to conclude the proof.

Proof of Theorem 1.4. The proof of this theorem is analogous to the proof of Theorem 1.1. Consider the chaotic expansion for the nodal length $\mathcal{N}_{E}$ in (3.52). For the 0-th chaotic component we have

$$
\mathcal{N}_{E}[0]=\mathbb{E}\left[\mathcal{N}_{E}\right]=\operatorname{area}(\mathcal{D}) \cdot 2 \pi^{2} E \cdot \beta_{0}^{2} \gamma_{0,0,0,0}=\operatorname{area}(\mathcal{D}) \pi E,
$$


where we used (3.53) and (3.55). By (4.61), (6.80) and Lemma 7.10 we deduce that, as $E \rightarrow+\infty$,

$$
\operatorname{Var}\left(\mathcal{N}_{E}\right) \sim \operatorname{Var}\left(\mathcal{N}_{E}[4]\right)
$$

and

$$
\frac{\mathcal{N}_{E}-\mathbb{E}\left[\mathcal{N}_{E}\right]}{\sqrt{\operatorname{Var}\left(\mathcal{N}_{E}\right.}}=\frac{\mathcal{N}_{E}[4]}{\sqrt{\operatorname{Var}\left(\mathcal{N}_{E}[4]\right.}}+o_{\mathbb{P}}(1),
$$

where $o_{\mathbb{P}}(1)$ denotes a sequence converging to zero in probability. Proposition 8.2 allows to conclude the proof.

Acknowledgements. The research leading to this work was supported by the Grant F1R-MTH-PUL-15CONFCONFLUENT (Ivan Nourdin), by the Grant F1R-MTH-PUL-15STAR-STARS (Giovanni Peccati and Maurizia Rossi) and by the FNR Grant R-AGR-3376-10 (Giovanni Peccati) at the University of Luxembourg. We are grateful to Massimo Notarnicola for a number of important remarks. We also thank an anonymous referee for a careful reading of our paper, and for useful suggestions.

Publisher's Note Springer Nature remains neutral with regard to jurisdictional claims in published maps and institutional affiliations.

\section{Appendix A}

Proof of Lemma 3.1. It is a standard fact that for any $m_{1}, m_{2}, n_{1}, n_{2} \in \mathbb{N}_{\geq 0}$

$$
\begin{aligned}
\mathbb{E}\left[\frac{\partial^{m_{1}+m_{2}}}{\partial x_{1}^{m_{1}} \partial x_{2}^{m_{2}}} B_{E}(x) \frac{\partial^{n_{1}+n_{2}}}{\partial y_{1}^{n_{1}} \partial y_{2}^{n_{2}}} B_{E}(y)\right] & =\frac{\partial^{m_{1}+m_{2}+n_{1}+n_{2}}}{\partial x_{1}^{m_{1}} \partial y_{1}^{n_{1}} \partial x_{2}^{m_{2}} \partial y_{2}^{n_{2}}} \mathbb{E}\left[B_{E}(x) B_{E}(y)\right] \\
& =\frac{\partial^{m_{1}+m_{2}+n_{1}+n_{2}}}{\partial x_{1}^{m_{1}} \partial y_{1}^{n_{1}} \partial x_{2}^{m_{2}} \partial y_{2}^{n_{2}}} r^{E}(x-y)
\end{aligned}
$$

where $r^{E}$ is defined as in (1.9). Let us first prove that for $x \in \mathbb{R}^{2}$, the covariance matrix of the centered Gaussian vector $\left(B_{E}(x), \nabla B_{E}(x)\right)$ is

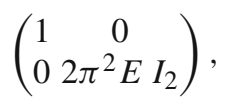

where $I_{2}$ denotes the $2 \times 2$-identity matrix. Recall from (3.44) that the following integral representation holds:

$$
J_{0}(2 \pi \sqrt{E}\|x\|)=\frac{1}{2 \pi} \int_{\mathbb{S}^{1}} \mathrm{e}^{i 2 \pi \sqrt{E}\langle\theta, x\rangle} d \theta, \quad x \in \mathbb{R}^{2},
$$

where $d \theta$ stands for the uniform measure on the unit circle. By (8.98) and (8.100), (8.99) immediately follows. Note now that, from (8.98), in particular we have

$$
\mathbb{E}\left[B_{E}(x) \partial_{1} B_{E}(y)\right]=-i \sqrt{E} \int_{\mathbb{S}^{1}} \theta_{1} \mathrm{e}^{2 \pi \sqrt{E} i\langle\theta, x-y\rangle} d \theta
$$


in order to find an explicit expression for (8.101), let us first compute $\int_{\mathbb{S}^{1}} \theta_{1} \mathrm{e}^{i r\langle\theta, u\rangle} d \theta$ for $r \in[0,+\infty)$ and any $u \in \mathbb{S}^{1}$. Let us denote by $r_{\tau}$ the rotation of angle $\tau$ (the latter is the angle between $\theta$ and $u$ ), then we have

$$
\begin{aligned}
\int_{\mathbb{S}^{1}} \theta_{1} e^{i r\langle\theta, u\rangle} d \theta & =\int_{-\pi}^{\pi}\left(r_{\tau}(u)\right)_{1} e^{i r \cos \tau} d \tau \\
& =\int_{-\pi}^{\pi}\left(\cos \tau u_{1}-\sin \tau u_{2}\right) e^{i r \cos \tau} d \tau \\
& =-\int_{-\pi}^{\pi}\left(\sin \tau u_{1}+\cos \tau u_{2}\right) e^{-i r \sin \tau} d \tau \\
& =-\int_{-\pi}^{\pi}\left(\sin \tau u_{1}+\cos \tau u_{2}\right)(\cos (r \sin \tau)-i \sin (r \sin \tau)) d \tau \\
& =-u_{2} \int_{-\pi}^{\pi} \cos \tau \cos (r \sin \tau) d \tau+i u_{1} \int_{-\pi}^{\pi} \sin \tau \sin (r \sin \tau) d \tau \\
& =-\pi u_{2}\left(J_{1}(r)+J_{-1}(r)\right)+i \pi u_{1}\left(J_{1}(r)-J_{-1}(r)\right) \\
& =2 i \pi u_{1} J_{1}(r),
\end{aligned}
$$

where we used integral representation formulas for $\alpha$-order Bessel functions of the first kind $J_{\alpha}[\mathrm{AS} 64, \S 9.1]$, so that, whenever $x \neq y$,

$$
\mathbb{E}\left[B_{E}(x) \partial_{1} B_{E}(y)\right]=2 \pi \sqrt{E} \frac{x_{1}-y_{1}}{\|x-y\|} J_{1}(2 \pi \sqrt{E}\|x-y\|) .
$$

Analogously, we get

$$
\mathbb{E}\left[B_{E}(x) \partial_{2} B_{E}(y)\right]=2 \pi \sqrt{E} \frac{x_{2}-y_{2}}{\|x-y\|} J_{1}(2 \pi \sqrt{E}\|x-y\|) ;
$$

(8.102) and (8.103) prove (3.32). For $k, l \in\{1,2\}$ from (8.98) and (3.44) we have

$$
\mathbb{E}\left[\partial_{k} B_{E}(x) \partial_{l} B_{E}(y)\right]=2 \pi E \int_{\mathbb{S}^{1}} z_{k} z_{l} e^{2 i \pi\langle z, \sqrt{E}(x-y)\rangle} d z .
$$

Let us first compute $\int_{\mathbb{S}^{1}} z_{1}^{2} e^{i r\langle z, u\rangle} d z$ for $(r, u) \in[0, \infty) \times \mathbb{S}^{1}$ : we have, again with $r_{\tau}$ denoting the rotation of angle $\tau$,

$$
\begin{aligned}
& \int_{\mathbb{S}^{1}} z_{1}^{2} e^{i r\langle z, u\rangle} d z=\int_{-\pi}^{\pi}\left(r_{\tau}(u)\right)_{1}^{2} e^{i r \cos \tau} d \tau \\
= & \int_{-\pi}^{\pi}\left(\cos \tau u_{1}-\sin \tau u_{2}\right)^{2} e^{i r \cos \tau} d \tau=\int_{-\pi}^{\pi}\left(\sin \tau u_{1}+\cos \tau u_{2}\right)^{2} e^{-i r \sin \tau} d \tau \\
= & \int_{-\pi}^{\pi}\left(\sin ^{2} \tau u_{1}^{2}+\cos ^{2} \tau u_{2}^{2}+2 \cos \tau \sin \tau u_{1} u_{2}\right)(\cos (r \sin \tau)-i \sin (r \sin \tau)) d \tau \\
= & \int_{-\pi}^{\pi}\left(\sin ^{2} \tau u_{1}^{2}+\cos ^{2} \tau u_{2}^{2}\right) \cos (r \sin \tau) d \tau-i u_{1} u_{2} \int_{-\pi}^{\pi} \sin (2 \tau) \sin (r \sin \tau) d \tau \\
= & \frac{1}{2} \int_{-\pi}^{\pi}\left(1+\left(1-2 u_{1}^{2}\right) \cos (2 \tau)\right) \cos (r \sin \tau) d \tau \\
= & \pi J_{0}(r)+\frac{\pi}{2}\left(1-2 u_{1}^{2}\right)\left(J_{2}(r)+J_{-2}(r)\right)=\pi J_{0}(r)+\left(1-2 u_{1}^{2}\right) \pi J_{2}(r) .
\end{aligned}
$$


Similarly

$$
\int_{\mathbb{S}^{1}} z_{2}^{2} e^{i r\langle z, u\rangle} d z=\pi J_{0}(r)+\left(1-2 u_{2}^{2}\right) \pi J_{2}(r)=\pi J_{0}(r)+\left(2 u_{1}^{2}-1\right) \pi J_{2}(r),
$$

whereas

$$
\begin{aligned}
& \int_{\mathbb{S}^{1}} z_{1} z_{2} e^{i r\langle z, u\rangle} d z=\int_{-\pi}^{\pi}\left(r_{\tau}(u)\right)_{1}\left(r_{\tau}(u)\right)_{2} e^{i r \cos \tau} d \tau \\
= & \int_{-\pi}^{\pi}\left(\cos \tau u_{1}-\sin \tau u_{2}\right)\left(\sin \tau u_{1}+\cos \tau u_{2}\right) e^{i r \cos \tau} d \tau \\
= & -\int_{-\pi}^{\pi}\left(\sin \tau u_{1}+\cos \tau u_{2}\right)\left(\cos \tau u_{1}-\sin \tau u_{2}\right) e^{-i r \sin \tau} d \tau \\
= & \int_{-\pi}^{\pi}\left(\frac{1}{2} \sin (2 \tau)\left(1-2 u_{1}^{2}\right)-\cos (2 \tau) u_{1} u_{2}\right)(\cos (r \sin \tau)-i \sin (r \sin \tau)) d \tau \\
= & -u_{1} u_{2} \int_{-\pi}^{\pi} \cos (2 \tau) \cos (r \sin \tau) d \tau \\
= & -u_{1} u_{2} \pi\left(J_{2}(r)+J_{-2}(r)\right)=-2 u_{1} u_{2} \pi J_{2}(r) .
\end{aligned}
$$

Thus, when $x \neq y$,

$$
\begin{aligned}
\mathbb{E}\left[\partial_{1} B_{E}(x) \partial_{1} B_{E}(y)\right]=2 & \pi^{2} E\left(J_{0}(2 \pi \sqrt{E}\|x-y\|)\right. \\
& \left.+\left(1-2 \frac{\left(x_{1}-y_{1}\right)^{2}}{\|x-y\|^{2}}\right) J_{2}(2 \pi \sqrt{E}\|x-y\|)\right) \\
\mathbb{E}\left[\partial_{2} B_{E}(x) \partial_{2} B_{E}(y)\right]=2 & \pi^{2} E\left(J_{0}(2 \pi \sqrt{E}\|x-y\|)\right. \\
& \left.+\left(1-2 \frac{\left(x_{2}-y_{2}\right)^{2}}{\|x-y\|^{2}}\right) J_{2}(2 \pi \sqrt{E}\|x-y\|)\right) \\
\mathbb{E}\left[\partial_{1} B_{E}(x) \partial_{2} B_{E}(y)\right]=- & 4 \pi^{2} E \frac{\left(x_{1}-y_{1}\right)\left(x_{2}-y_{2}\right)}{\|x-y\|^{2}} J_{2}(2 \pi \sqrt{E}\|x-y\|),
\end{aligned}
$$

which are (3.33) and (3.34).

The following result concerns some (known) properties of the nodal sets of $B_{E}$ and its complex version.

Lemma 8.4. 1. The value 0 is not singular for $B_{E}$ a.s., i.e.

$$
\mathbb{P}\left(\exists x: B_{E}(x)=0, \nabla B_{E}(x)=0\right)=0
$$

2. the nodal set $B_{E}^{-1}(0)$ is a smooth one dimensional submanifold of $\mathbb{R}^{2}$ a.s.;

3. $B_{E}^{-1}(0) \cap \partial \mathcal{D}$ consists of a finite number of points a.s.;

4. the nodal set $\left(B_{E}^{\mathbb{C}}\right)^{-1}(0)=B_{E}^{-1}(0) \cap \widehat{B}_{E}^{-1}(0)$ consists of isolated points a.s.;

5. the number of nodal points $\left(B_{E}^{\mathbb{C}}\right)^{-1}(0)$ in $\mathcal{D}$ is a.s. finite and none of them lies on $\partial D$ a.s. 
Proof. 1. Proposition 6.12 in [AW] ensures that 0 is not a singular value a.s. Indeed, the hypothesis of Proposition 6.12 are satisfied, the random variables $B_{E}(x), \partial_{1} B_{E}(x)$, $\partial_{2} B_{E}(x)$ being independent for fixed $x \in \mathbb{R}^{2}$ (Point 2. in Lemma 3.1).

2. It follows from Point 1 by Sard's lemma.

3. Let $\gamma$ be a unit speed parameterization of the boundary $\partial \mathcal{D}$. The restriction of $B_{E}$ to $\partial \mathcal{D}$ is the one-dimensional Gaussian process $t \mapsto B_{E}(\gamma(t))$ whose first time-derivative is

$$
B_{E}(\gamma(t))^{\prime}=\left\langle\nabla B_{E}(\gamma(t)), \dot{\gamma}(t)\right\rangle .
$$

From (8.104) and the arguments used in the proof of Point 1. We deduce that

$$
\mathbb{P}\left(\exists t: B_{E}(\gamma(t))=B_{E}(\gamma(t))^{\prime}=0\right)=0,
$$

i.e. the value 0 is not singular a.s. for $B_{E}(\gamma)$, hence the zeros of $B_{E}$ on $\partial \mathcal{D}$ are isolated points a.s. (by a standard application of the inverse mapping theorem), and their number is finite (see [AT, p.269]).

4. Let us consider the two-dimensional Gaussian field on the plane $\left(B_{E}, \widehat{B}_{E}\right)$, where we recall $\widehat{B}_{E}$ to be an independent copy of $B_{E}$. In view of Point 1 ., the value $(0,0)$ is not singular for $\left(B_{E}, \widehat{B}_{E}\right)$, hence a standard application of the inverse mapping theorem entails that the common zeros of $B_{E}$ and $\widehat{B}_{E}$ are isolated points.

5. The value 0 being not singular for $\left(B_{E}, \widehat{B}_{E}\right)$, from [AT, p.269] the number of nodal points in $\mathcal{D}$ is finite a.s. We can apply Lemma 11.2.10 in [AT] to the two-dimensional random field $\left(B_{E}, \widehat{B}_{E}\right)$ restricted to the boundary $\partial \mathcal{D}$ to get that $\left(B_{E}^{\mathbb{C}}\right)^{-1}(0) \cap \partial \mathcal{D}=\emptyset$ a.s.

Proof of Lemma 3.2. We can rewrite (3.37) by means of the co-area formula [AW, Proposition 6.13] as

$$
\mathcal{L}_{E}^{\varepsilon}=\frac{1}{2 \varepsilon} \int_{-\varepsilon}^{\varepsilon} \operatorname{length}\left(B_{E}^{-1}(s) \cap \mathcal{D}\right) d s,
$$

where $B_{E}^{-1}(s)=\left\{x \in \mathbb{R}^{2}: B_{E}(x)=s\right\}$. Theorem 3 in [APP16] ensures that the map $s \mapsto$ length $\left(B_{E}^{-1}(s)\right)$ is a.s. continuous at 0 , so that by the Foundamental Theorem of Calculus we have

$$
\lim _{\varepsilon \rightarrow 0} \mathcal{L}_{E}^{\varepsilon}=\lim _{\varepsilon \rightarrow 0} \frac{1}{2 \varepsilon} \int_{-\varepsilon}^{\varepsilon} \text { length }\left(B_{E}^{-1}(s) \cap \mathcal{D}\right) d s=\mathcal{L}_{E}, \quad \text { a.s. }
$$

In order to prove (3.40) we apply Theorem 11.2.3 in [AT], the hypothesis being satisfied.

Proof of Lemma 3.3. We have $\mathcal{L}_{E} \in L^{2}(\mathbb{P})$, the nodal length of $B_{E}$ being a.s. bounded in $\mathcal{D}$ [DF88]. The collection of random variables $\left\{\mathcal{L}_{E}^{\varepsilon}\right\}_{\varepsilon>0}$ is in $L^{2}(\mathbb{P})$ since

$$
\mathcal{L}_{E}^{\varepsilon} \leq \frac{1}{2 \varepsilon} \int_{\mathcal{D}}\left\|\nabla B_{E}(x)\right\| d x,
$$

hence

$$
\begin{aligned}
\mathbb{E}\left[\left(\mathcal{L}_{E}^{\varepsilon}\right)^{2}\right] & \leq \frac{1}{4 \varepsilon^{2}} \int_{(\mathcal{D})^{2}} \mathbb{E}\left[\left\|\nabla B_{E}(x)\right\| \cdot\left\|\nabla B_{E}(y)\right\|\right] d x d y \\
& \leq \operatorname{area}(\mathcal{D}) \frac{1}{4 \varepsilon^{2}} \int_{\mathcal{D}} \mathbb{E}\left[\left\|\nabla B_{E}(x)\right\|^{2}\right] d x=(\operatorname{area}(\mathcal{D}))^{2} \frac{\pi^{2} E}{\varepsilon^{2}}<+\infty .
\end{aligned}
$$


In view of Lemma 3.2, in order to prove that $\mathcal{L}_{E}^{\varepsilon}$ converges to the nodal length in $L^{2}(\mathbb{P})$ it suffices to show that

$$
\lim _{\varepsilon \rightarrow 0} \mathbb{E}\left[\left(\mathcal{L}_{E}^{\varepsilon}\right)^{2}\right]=\mathbb{E}\left[\mathcal{L}_{E}^{2}\right]
$$

(see also [Ros15, Lemma 7.2.1]). By Fatou's lemma and (8.105) we get

$$
\mathbb{E}\left[\mathcal{L}_{E}^{2}\right] \leq \liminf _{\varepsilon \rightarrow 0} \mathbb{E}\left[\left(\mathcal{L}_{E}^{\varepsilon}\right)^{2}\right] \leq \limsup _{\varepsilon \rightarrow 0} \mathbb{E}\left[\left(\frac{1}{2 \varepsilon} \int_{-\varepsilon}^{\varepsilon} \mathcal{L}_{E}(s) d s\right)^{2}\right]
$$

By Jensen's inequality

$$
\mathbb{E}\left[\mathcal{L}_{E}^{2}\right] \leq \limsup _{\varepsilon \rightarrow 0} \mathbb{E}\left[\left(\frac{1}{2 \varepsilon} \int_{-\varepsilon}^{\varepsilon} \mathcal{L}_{E}(s) d s\right)^{2}\right] \leq \limsup _{\varepsilon \rightarrow 0} \frac{1}{2 \varepsilon} \int_{-\varepsilon}^{\varepsilon} \mathbb{E}\left[\mathcal{L}_{E}(s)^{2}\right] d s=\mathbb{E}\left[\mathcal{L}_{E}^{2}\right],
$$

the last step following from the continuity of the map $s \mapsto \mathbb{E}\left[\mathcal{L}_{E}(s)^{2}\right]$ at 0 which will be proven just below. Standard Kac-Rice formula [AW, Theorem 6.9] allows to write

$$
\begin{aligned}
& \mathbb{E}\left[\mathcal{L}_{E}(s)^{2}\right] \\
& =\int_{(\mathcal{D})^{2}} \mathbb{E}\left[\left\|\nabla B_{E}(x)\right\|\left\|\nabla B_{E}(y)\right\| \mid B_{E}(x)=s, B_{E}(y)=s\right] p_{\left(B_{E}(x), B_{E}(y)\right)}(s, s) d x d y,
\end{aligned}
$$

where $p_{\left(B_{E}(x), B_{E}(y)\right)}$ denotes the density of the random vector $\left(B_{E}(x), B_{E}(y)\right)$. It suffices to show that, for $\delta>0$, there exists a measurable function $g=g(x, y)$ integrable on $(\mathcal{D})^{2}$ such that

$$
\begin{aligned}
& \mathbb{E}\left[\left\|\nabla B_{E}(x)\right\|\left\|\nabla B_{E}(y)\right\| \mid B_{E}(x)=s, B_{E}(y)=s\right] p_{\left(B_{E}(x), B_{E}(y)\right)}(s, s) \\
& \quad \leq g(x, y), \quad \forall s \in[-\delta, \delta] .
\end{aligned}
$$

It is immediate that

$$
p_{\left(B_{E}(x), B_{E}(y)\right)}(s, s) \leq p_{\left(B_{E}(x), B_{E}(y)\right)}(0,0)=\frac{1}{2 \pi \sqrt{1-J_{0}(2 \pi \sqrt{E}\|x-y\|)^{2}}} .
$$

From Lemma 3.1, the vector $\nabla B_{E}(x)$ conditioned to $B_{E}(x)=B_{E}(y)=s$ is Gaussian with mean

$$
s \frac{\nabla_{x} r^{E}(x-y)}{1+r^{E}(x-y)}
$$

and covariance matrix

$$
\begin{aligned}
& \Omega^{E}(x-y) \\
& \quad:=2 \pi^{2} E I_{2}-\frac{1}{1-r^{E}(x-y)^{2}} \\
& \quad \times\left(\begin{array}{cc}
\left(\partial_{x_{1}} r^{E}(x-y)\right)^{2} & \partial_{x_{1}} r^{E}(x-y) \partial_{x_{2}} r^{E}(x-y) \\
\partial_{x_{1}} r^{E}(x-y) \partial_{x_{2}} r^{E}(x-y) & \left(\partial_{x_{2}} r^{E}(x-y)\right)^{2}
\end{array}\right) .
\end{aligned}
$$


Jensen's inequality yields

$$
\begin{aligned}
\mathbb{E} & {\left[\left\|\nabla B_{E}(x)\right\|\left\|\nabla B_{E}(y)\right\| \mid B_{E}(x)=s, B_{E}(y)=s\right] } \\
\leq & \mathbb{E}\left[\left\|\nabla B_{E}(x)\right\|^{2} \mid B_{E}(x)=s, B_{E}(y)=s\right] \\
= & \operatorname{Var}\left(\partial_{1} B_{E}(x) \mid B_{E}(x)=s, B_{E}(y)=s\right) \\
+ & \operatorname{Var}\left(\partial_{2} B_{E}(x) \mid B_{E}(x)=s, B_{E}(y)=s\right) \\
+ & \mathbb{E}\left[\partial_{1} B_{E}(x) \mid B_{E}(x)=s, B_{E}(y)=s\right]^{2}+\mathbb{E}\left[\partial_{2} B_{E}(x) \mid B_{E}(x)=s, B_{E}(y)=s\right]^{2} \\
= & 4 \pi^{2} E-\frac{4 \pi^{2} E J_{1}(2 \pi \sqrt{E}\|x-y\|)^{2}}{1-J_{0}(2 \pi \sqrt{E}\|x-y\|)^{2}} \\
& +s^{2} \frac{4 \pi^{2} E J_{1}(2 \pi \sqrt{E}\|x-y\|)^{2}}{\left(1+J_{0}(2 \pi \sqrt{E}\|x-y\|)\right)^{2}} \\
& \leq 2 \pi^{2} E+s^{2} \frac{4 \pi^{2} E J_{1}(2 \pi \sqrt{E}\|x-y\|)^{2}}{\left(1+J_{0}(2 \pi \sqrt{E}\|x-y\|)\right)^{2}} \\
\leq & 2 \pi^{2} E+\delta^{2} \frac{4 \pi^{2} E J_{1}(2 \pi \sqrt{E}\|x-y\|)^{2}}{\left(1+J_{0}(2 \pi \sqrt{E}\|x-y\|)\right)^{2}} .
\end{aligned}
$$

If we set

$$
g(x, y):=\frac{1}{2 \pi \sqrt{1-J_{0}(2 \pi \sqrt{E}\|x-y\|)^{2}}}\left(2 \pi^{2} E+\delta^{2} \frac{4 \pi^{2} E J_{1}(2 \pi \sqrt{E}\|x-y\|)^{2}}{\left(1+J_{0}(2 \pi \sqrt{E}\|x-y\|)\right)^{2}}\right),
$$

then the proof of (3.41) is concluded.

The proof of (3.42) relies on the same argument as that of (3.41). Let us first show that $\mathcal{N}_{E} \in L^{2}(\mathbb{P})$. Theorem 6.3 in $[\mathrm{AW}]$ ensures that the second factorial moment of $\mathcal{N}_{E}$ has the following integral representation

$$
\begin{aligned}
\mathbb{E} & {\left[\mathcal{N}_{E}\left(\mathcal{N}_{E}-1\right)\right] } \\
= & \int_{(\mathcal{D})^{2}} \mathbb{E}\left[\left|\operatorname{Jac}_{B_{E}, \widehat{B}_{E}}(x)\right|\left|\mathrm{Jac}_{B_{E}, \widehat{B}_{E}}(y)\right| \mid\right. \\
& \left.B_{E}(x)=0, B_{E}(y)=0, \widehat{B}_{E}(x)=0, \widehat{B}_{E}(y)=0\right] \\
& \times p_{\left(B_{E}(x), B_{E}(y)\right)}(0,0) d x d y .
\end{aligned}
$$

Reasoning as in the proof of [DNPR16, Lemma 3.4], we have

$$
\begin{aligned}
& \mathbb{E}\left[\left|\operatorname{Jac}_{B_{E}, \widehat{B}_{E}}(x)\right| \mid \mathrm{Jac}_{B_{E}, \widehat{B}_{E}}(y) \| B_{E}(x)=0, B_{E}(y)=0,\right. \\
& \left.\widehat{B}_{E}(x)=0, \widehat{B}_{E}(y)=0\right] \\
& \ll \frac{\operatorname{det}\left(\Omega^{E}(x-y)\right)}{1-J_{0}(2 \pi \sqrt{E}\|x-y\|)^{2}},
\end{aligned}
$$

where, for any $s \in \mathbb{R}, \Omega^{E}(x-y)$ denotes the covariance matrix of $\nabla B_{E}(x)$ conditioned to $B_{E}(x)=B_{E}(y)=s$. Lemma 8.20 ensures that the double integral over $\mathcal{D}$ of the rhs of (8.111) is finite. 
Let us now prove that the map $s \mapsto \mathbb{E}\left[\mathcal{N}_{E}(s)^{2}\right]$ is continuous at 0 . Note first that we can write

$$
\mathbb{E}\left[\mathcal{N}_{E}(s)^{2}\right]=\mathbb{E}\left[\mathcal{N}_{E}(s)\left(\mathcal{N}_{E}(s)-1\right)\right]+\mathbb{E}\left[\mathcal{N}_{E}(s)\right]
$$

To evaluate the mean, we use Kac-Rice formula [AW, Thereom 6.2] and Lemma 3.1

$$
\mathbb{E}\left[\mathcal{N}_{E}(s)\right]=\int_{\mathcal{D}} \mathbb{E}\left[\left|\operatorname{Jac}_{B_{E}, \widehat{B}_{E}}(x)\right|\right] p_{\left(B_{E}(x), \widehat{B}_{E}(x)\right)}(s, s) d x .
$$

Since $\mathbb{E}\left[\left|\operatorname{Jac}_{B_{E}, \widehat{B}_{E}}(x)\right|\right]=2 \pi^{2} E$ and $p_{\left(B_{E}(x), \widehat{B}_{E}(x)\right)}(s, s) \leq \frac{1}{2 \pi}$ for every $s$, then $s \mapsto$ $\mathbb{E}\left[\mathcal{N}_{E}(s)\right]$ is continuous.

Let us now deal with the second factorial moment, again using Kac-Rice formula [AW, Theorem 6.3].

$$
\begin{aligned}
\mathbb{E} & {\left[\mathcal{N}_{E}(s)\left(\mathcal{N}_{E}(s)-1\right)\right] } \\
= & \int_{(\mathcal{D})^{2}} \mathbb{E}\left[\left|\operatorname{Jac}_{B_{E}, \widehat{B}_{E}}(x)\right|\left|\operatorname{Jac}_{B_{E}, \widehat{B}_{E}}(y)\right| \mid B_{E}(x)=\widehat{B}_{E}(x)=B_{E}(y)=\widehat{B}_{E}(y)=s\right] \\
& \times p_{\left(B_{E}(x), \widehat{B}_{E}(x), B_{E}(y), \widehat{B}_{E}(y)\right)}(s, s, s, s) d x d y .
\end{aligned}
$$

Jensen's inequality yields

$$
\begin{gathered}
\mathbb{E}\left[|\operatorname{Jac}(x)||\operatorname{Jac}(y)| \mid B_{E}(x)=\widehat{B}_{E}(x)=B_{E}(y)=\widehat{B}_{E}(y)=s\right] \\
\leq \mathbb{E}\left[|\operatorname{Jac}(x)|^{2} \mid B_{E}(x)=\widehat{B}_{E}(x)=B_{E}(y)=\widehat{B}_{E}(y)=s\right] \\
\quad=2\left(\mathbb{E}\left[X^{2}\right] \mathbb{E}\left[Y^{2}\right]-\mathbb{E}[X Y]^{2}\right),
\end{gathered}
$$

where $(X, Y)$ is a random vector with the same distribution as $\nabla B_{E}(x) \mid B_{E}(x)=$ $B_{E}(y)=s$. Hence some straightforward computations lead to

$$
\begin{aligned}
\mathbb{E}\left[X^{2}\right] \mathbb{E}\left[Y^{2}\right]-\mathbb{E}[X Y]^{2} & =2 \pi^{2} E\left(2 \pi^{2} E-\frac{\left(\partial_{x_{1}} r^{E}(x-y)\right)^{2}+\left(\partial_{x_{2}} r^{E}(x-y)\right)^{2}}{1-r^{E}(x-y)^{2}}\right) \\
& +2 \pi^{2} E s^{2} \frac{\left(\partial_{x_{1}} r^{E}(x-y)\right)^{2}+\left(\partial_{x_{2}} r^{E}(x-y)\right)^{2}}{\left(1+r^{E}(x-y)\right)^{2}} \\
& \leq 2 \pi^{2} E\left(2 \pi^{2} E-\frac{\left(\partial_{x_{1}} r^{E}(x-y)\right)^{2}+\left(\partial_{x_{2}} r^{E}(x-y)\right)^{2}}{1-r^{E}(x-y)^{2}}\right) \\
& +2 \pi^{2} E \delta^{2} \frac{\left(\partial_{x_{1}} r^{E}(x-y)\right)^{2}+\left(\partial_{x_{2}} r^{E}(x-y)\right)^{2}}{\left(1+r^{E}(x-y)\right)^{2}},
\end{aligned}
$$

for $s \in[-\delta, \delta]$, which is integrable on $\mathcal{D} \times \mathcal{D}$.

\section{Appendix B}

Lemma 8.5. As $E \rightarrow+\infty$, we have

(i) $\operatorname{Var}\left(a_{1, E}\right)=24 \int_{\mathcal{D}} \int_{\mathcal{D}} r^{E}(x-y)^{4} d x d y \sim 9 \frac{\operatorname{area}(\mathcal{D})}{\pi^{3}} \times \frac{\log E}{E}$, 


$$
\begin{aligned}
& \operatorname{Cov}\left(a_{1, E}, a_{2, E}\right)=24 \int_{\mathcal{D}} \int_{\mathcal{D}} \widetilde{r}_{0,1}^{E}(x-y)^{4} d x d y \sim \frac{27}{2} \frac{\operatorname{area}(\mathcal{D})}{\pi^{3}} \times \frac{\log E}{E}, \\
& \operatorname{Cov}\left(a_{1, E}, a_{3, E}\right)=24 \int_{\mathcal{D}} \int_{\mathcal{D}} \widetilde{r}_{0,2}^{E}(x-y)^{4} d x d y \sim \frac{27}{2} \frac{\operatorname{area}(\mathcal{D})}{\pi^{3}} \times \frac{\log E}{E}, \\
& \operatorname{Cov}\left(a_{1, E}, a_{3, E}\right)=24 \int_{\mathcal{D}} \int_{\mathcal{D}} \widetilde{r}_{0,1}^{E}(x-y)^{2} \widetilde{r}_{0,2}^{E}(x-y)^{2} d x d y \sim \frac{9}{2} \frac{\operatorname{area}(\mathcal{D})}{\pi^{3}} \times \frac{\log E}{E}, \\
& \operatorname{Cov}\left(a_{1, E}, a_{5, E}\right)=24 \int_{\mathcal{D}} \int_{\mathcal{D}} r^{E}(x-y)^{2} \widetilde{r}_{0,1}^{E}(x-y)^{2} d x d y \sim 3 \frac{\operatorname{area}(\mathcal{D})}{\pi^{3}} \times \frac{\log E}{E}, \\
& \operatorname{Cov}\left(a_{1, E}, a_{6, E}\right)=24 \int_{\mathcal{D}} \int_{\mathcal{D}} r^{E}(x-y)^{2} \widetilde{r}_{0,2}^{E}(x-y)^{2} d x d y \sim 3 \frac{\operatorname{area}(\mathcal{D})}{\pi^{3}} \times \frac{\log E}{E} .
\end{aligned}
$$

Proof. Let us prove (i). From Proposition 5.2,

$$
\begin{aligned}
\operatorname{Var}\left(a_{1, E}\right)= & 24 \int_{\mathcal{D}} \int_{\mathcal{D}} r^{E}(x-y)^{4} d x d y \\
= & 24 \operatorname{area}(\mathcal{D}) \frac{2 \pi}{E} \int_{1}^{\sqrt{E} \cdot \operatorname{diam}(\mathcal{D})} \\
& \times \psi\left(\frac{1}{\pi \sqrt{\psi}} \cos \left(2 \pi \psi-\frac{\pi}{4}\right)\right)^{4} d \psi+O\left(\frac{1}{E}\right) \\
= & 24 \operatorname{area}(\mathcal{D}) \frac{2}{\pi^{3} E} \int_{1}^{\sqrt{E} \cdot \operatorname{diam}(\mathcal{D})} \frac{1}{\psi} \\
& \times \cos ^{4}\left(2 \pi \psi-\frac{\pi}{4}\right) d \psi+O\left(\frac{1}{E}\right) .
\end{aligned}
$$

Thanks to (6.78) we have that, as $E \rightarrow+\infty$,

$$
\begin{aligned}
& \text { 24 } \operatorname{area}(\mathcal{D}) \frac{2}{\pi^{3} E} \int_{1}^{\sqrt{E} \cdot \operatorname{diam}(\mathcal{D})} \frac{1}{\psi} \cos ^{4}\left(2 \pi \psi-\frac{\pi}{4}\right) d \psi \\
& \sim 24 \operatorname{area}(\mathcal{D}) \frac{2}{\pi^{3} E} \cdot \frac{3}{8} \cdot \log \sqrt{E} \\
& =\frac{9}{\pi^{3} E} \operatorname{area}(\mathcal{D}) \log E,
\end{aligned}
$$

that allows to conclude. The proof for the remaining terms is analogous to the proof of (i), and hence omitted.

The proofs of the following lemmas follow from an application of Proposition 5.2, completely analogous to the one appearing in the proof of Lemma 8.5.

Lemma 8.6. As $E \rightarrow+\infty$, we have

$$
\begin{aligned}
\operatorname{Var}\left(a_{2, E}\right) & =24 \int_{\mathcal{D}} \int_{\mathcal{D}} \widetilde{r}_{1,1}^{E}(x-y)^{4} d x d y \sim \frac{315}{8} \frac{\operatorname{area}(\mathcal{D})}{\pi^{3}} \times \frac{\log E}{E}, \\
\operatorname{Cov}\left(a_{2, E}, a_{3, E}\right) & =24 \int_{\mathcal{D}} \int_{\mathcal{D}} \widetilde{r}_{1,2}^{E}(x-y)^{4} d x d y \sim \frac{27}{8} \frac{\operatorname{area}(\mathcal{D})}{\pi^{3}} \times \frac{\log E}{E}, \\
\operatorname{Cov}\left(a_{2, E}, a_{4, E}\right) & =24 \int_{\mathcal{D}} \int_{\mathcal{D}} \widetilde{r}_{1,1}^{E}(x-y)^{2} \widetilde{r}_{1,2}^{E}(x-y)^{2} d x d y \sim \frac{45}{8} \frac{\operatorname{area}(\mathcal{D})}{\pi^{3}} \times \frac{\log E}{E},
\end{aligned}
$$


$\operatorname{Cov}\left(a_{2, E}, a_{5, E}\right)=24 \int_{\mathcal{D}} \int_{\mathcal{D}} \widetilde{r}_{0,1}^{E}(x-y)^{2} \widetilde{r}_{1,1}^{E}(x-y)^{2} d x d y \sim \frac{15}{2} \frac{\operatorname{area}(\mathcal{D})}{\pi^{3}} \times \frac{\log E}{E}$,

$\operatorname{Cov}\left(a_{2, E}, a_{6, E}\right)=24 \int_{\mathcal{D}} \int_{\mathcal{D}} \widetilde{r}_{0,1}^{E}(x-y)^{2} \widetilde{r}_{1,2}^{E}(x-y)^{2} d x d y \sim \frac{3}{2} \frac{\operatorname{area}(\mathcal{D})}{\pi^{3}} \times \frac{\log E}{E}$.

Lemma 8.7. As $E \rightarrow+\infty$, we have

$$
\begin{aligned}
\operatorname{Var}\left(a_{3, E}\right)= & 24 \int_{\mathcal{D}} \int_{\mathcal{D}} \widetilde{r}_{2,2}^{E}(x-y)^{4} d x d y \sim \frac{315}{8} \frac{\operatorname{area}(\mathcal{D})}{\pi^{3}} \times \frac{\log E}{E} \\
\operatorname{Cov}\left(a_{3, E}, a_{4, E}\right)= & 24 \int_{\mathcal{D}} \int_{\mathcal{D}} \widetilde{r}_{2,2}^{E}(x-y)^{2} \widetilde{r}_{1,2}^{E}(x-y)^{2} d x d y \\
& \sim \frac{45}{8} \frac{\operatorname{area}(\mathcal{D})}{\pi^{3}} \times \frac{\log E}{E} \\
\operatorname{Cov}\left(a_{3, E}, a_{5, E}\right)= & 24 \int_{\mathcal{D}} \int_{\mathcal{D}} \widetilde{r}_{0,2}^{E}(x-y)^{2} \widetilde{r}_{1,2}^{E}(x-y)^{2} d x d y \\
& \sim \frac{3}{2} \frac{\operatorname{area}(\mathcal{D})}{\pi^{3}} \times \frac{\log E}{E} \\
\operatorname{Cov}\left(a_{3, E}, a_{6, E}\right)= & 24 \int_{\mathcal{D}} \int_{\mathcal{D}} \widetilde{r}_{0,2}^{E}(x-y)^{2} \widetilde{r}_{2,2}^{E}(x-y)^{2} d x d y \\
& \sim \frac{15}{2} \frac{\operatorname{area}(\mathcal{D})}{\pi^{3}} \times \frac{\log E}{E} .
\end{aligned}
$$

Lemma 8.8. As $E \rightarrow+\infty$, we have

$$
\begin{aligned}
\operatorname{Var}\left(a_{4, E}\right)= & 4 \int_{\mathcal{D}} \int_{\mathcal{D}}\left(\widetilde{r}_{1,1}^{E}(x-y)^{2} \widetilde{r}_{2,2}^{E}(x-y)^{2}+\widetilde{r}_{1,2}^{E}(x-y)^{4}\right. \\
& \left.+4 \widetilde{r}_{1,1}^{E}(x-y) \widetilde{r}_{2,2}^{E}(x-y) \widetilde{r}_{1,2}^{E}(x-y)^{2}\right) d x d y \\
& \sim \frac{27}{8} \frac{\operatorname{area}(\mathcal{D})}{\pi^{3}} \times \frac{\log E}{E}, \\
\operatorname{Cov}\left(a_{4, E}, a_{5, E}\right)= & 4 \int_{\mathcal{D}} \int_{\mathcal{D}}\left(\widetilde{r}_{0,1}^{E}(x-y)^{2} \widetilde{r}_{1,2}^{E}(x-y)^{2}+\widetilde{r}_{0,2}^{E}(x-y)^{2} \widetilde{r}_{1,1}^{E}(x-y)^{2}\right. \\
& \left.+4 \widetilde{r}_{0,1}^{E}(x-y) \widetilde{r}_{0,2}^{E}(x-y) \widetilde{r}_{1,1}^{E}(x-y) \widetilde{r}_{1,2}^{E}(x-y)\right) d x d y \\
& \sim \frac{3}{2} \frac{\operatorname{area}(\mathcal{D})}{\pi^{3}} \times \frac{\log E}{E}, \\
\operatorname{Cov}\left(a_{4, E}, a_{6, E}\right)= & 4 \int_{\mathcal{D}} \int_{\mathcal{D}}\left(\widetilde{r}_{0,1}^{E}(x-y)^{2} \widetilde{r}_{2,2}^{E}(x-y)^{2}+\widetilde{r}_{0,2}^{E}(x-y)^{2} \widetilde{r}_{1,2}^{E}(x-y)^{2}\right. \\
& \left.+4 \widetilde{r}_{0,1}^{E}(x-y) \widetilde{r}_{0,2}^{E}(x-y) \widetilde{r}_{2,2}^{E}(x-y) \widetilde{r}_{1,2}^{E}(x-y)\right) d x d y \\
& \sim \frac{3}{2} \frac{\operatorname{area}(\mathcal{D})}{\pi^{3}} \times \frac{\log E}{E} .
\end{aligned}
$$


Lemma 8.9. As $E \rightarrow+\infty$, we have

$$
\begin{aligned}
\operatorname{Var}\left(a_{5, E}\right) & =4 \int_{\mathcal{D}} \int_{\mathcal{D}}\left(r^{E}(x-y)^{2} \widetilde{r}_{1,1}^{E}(x-y)^{2}+\widetilde{r}_{0,1}^{E}(x-y)^{4}\right. \\
& \left.-4 r^{E}(x-y) \widetilde{r}_{1,1}^{E}(x-y) \widetilde{r}_{0,1}^{E}(x-y)^{2}\right) d x d y \sim \frac{3}{2} \frac{\operatorname{area}(\mathcal{D})}{\pi^{3}} \times \frac{\log E}{E}, \\
\operatorname{Cov}\left(a_{5, E}, a_{6, E}\right) & =4 \int_{\mathcal{D}} \int_{\mathcal{D}}\left(r^{E}(x-y)^{2} \widetilde{r}_{2,2}^{E}(x-y)^{2}+\widetilde{r}_{0,2}^{E}(x-y)^{4}\right. \\
& \left.-4 r^{E}(x-y) \widetilde{r}_{0,2}^{E}(x-y)^{2} \widetilde{r}_{2,2}^{E}(x-y)\right) d x d y \sim \frac{1}{2} \frac{\operatorname{area}(\mathcal{D})}{\pi^{3}} \times \frac{\log E}{E} .
\end{aligned}
$$

Lemma 8.10. As $E \rightarrow+\infty$, we have

$$
\begin{aligned}
\operatorname{Var}\left(a_{6, E}\right) & =4 \int_{\mathcal{D}} \int_{\mathcal{D}}\left(r^{E}(x-y)^{2} \widetilde{r}_{2,2}^{E}(x-y)^{2}+\widetilde{r}_{0,2}^{E}(x-y)^{4}\right. \\
& \left.-4 r^{E}(x-y) \widetilde{r}_{2,2}^{E}(x-y) \widetilde{r}_{0,2}^{E}(x-y)^{2}\right) d x d y \sim \frac{3}{2} \frac{\operatorname{area}(\mathcal{D})}{\pi^{3}} \times \frac{\log E}{E} .
\end{aligned}
$$

Lemma 8.11. As $E \rightarrow+\infty$, we have

$$
\operatorname{Var}\left(b_{1, E}\right)=4 \int_{\mathcal{D}} \int_{\mathcal{D}} r^{E}(x-y)^{4}(u) d x d y \sim \frac{3}{8} \frac{\operatorname{area}(\mathcal{D})}{\pi^{3}} \times \frac{\log E}{E},
$$

$\operatorname{Cov}\left(b_{1, E}, b_{2, E}\right)=4 \int_{\mathcal{D}} \int_{\mathcal{D}} r^{E}(x-y)^{2} \widetilde{r}_{0,1}^{E}(x-y)^{2} d x d y \sim \frac{1}{8} \frac{\operatorname{area}(\mathcal{D})}{\pi^{3}} \times \frac{\log E}{E}$,

$\operatorname{Cov}\left(b_{1, E}, b_{3, E}\right)=4 \int_{\mathcal{D}} \int_{\mathcal{D}} r^{E}(x-y)^{2} \widetilde{r}_{0,2}^{E}(x-y)^{2} d x d y \sim \frac{1}{8} \frac{\operatorname{area}(\mathcal{D})}{\pi^{3}} \times \frac{\log E}{E}$,

$\operatorname{Cov}\left(b_{1, E}, b_{4, E}\right)=4 \int_{\mathcal{D}} \int_{\mathcal{D}} r^{E}(x-y)^{2} \widetilde{r}_{0,1}^{E}(x-y)^{2} d x d y \sim \frac{1}{8} \frac{\operatorname{area}(\mathcal{D})}{\pi^{3}} \times \frac{\log E}{E}$

$\operatorname{Cov}\left(b_{1, E}, b_{5, E}\right)=4 \int_{\mathcal{D}} \int_{\mathcal{D}} r^{E}(x-y)^{2} \widetilde{r}_{0,2}^{E}(x-y)^{2} d x d y \sim \frac{1}{8} \frac{\operatorname{area}(\mathcal{D})}{\pi^{3}} \times \frac{\log E}{E}$,

$\operatorname{Cov}\left(b_{1, E}, b_{6, E}\right)=4 \int_{\mathcal{D}} \int_{\mathcal{D}} \widetilde{r}_{0,1}^{E}(x-y)^{4} d x d y \sim \frac{9}{16} \frac{\operatorname{area}(\mathcal{D})}{\pi^{3}} \times \frac{\log E}{E}$,

$\operatorname{Cov}\left(b_{1, E}, b_{7, E}\right)=4 \int_{\mathcal{D}} \int_{\mathcal{D}} \widetilde{r}_{0,2}^{E}(x-y)^{4} d x d y \sim \frac{9}{16} \frac{\operatorname{area}(\mathcal{D})}{\pi^{3}} \times \frac{\log E}{E}$,

$\operatorname{Cov}\left(b_{1, E}, b_{8, E}\right)=4 \int_{\mathcal{D}} \int_{\mathcal{D}} \widetilde{r}_{0,1}^{E}(x-y)^{2} \widetilde{r}_{0,2}^{E}(x-y)^{2} d x d y \sim \frac{3}{16} \frac{\operatorname{area}(\mathcal{D})}{\pi^{3}} \times \frac{\log E}{E}$,

$\operatorname{Cov}\left(b_{1, E}, b_{9, E}\right)=4 \int_{\mathcal{D}} \int_{\mathcal{D}} \widetilde{r}_{0,1}^{E}(x-y)^{2} \widetilde{r}_{0,2}^{E}(x-y)^{2} d x d y \sim \frac{3}{16} \frac{\operatorname{area}(\mathcal{D})}{\pi^{3}} \times \frac{\log E}{E}$,

$\operatorname{Cov}\left(b_{1, E}, b_{10, E}\right)=4 \int_{\mathcal{D}} \int_{\mathcal{D}} \widetilde{r}_{0,1}^{E}(x-y)^{2} \widetilde{r}_{0,2}^{E}(x-y)^{2} d x d y \sim \frac{3}{16} \frac{\operatorname{area}(\mathcal{D})}{\pi^{3}} \times \frac{\log E}{E}$.

Lemma 8.12. As $E \rightarrow+\infty$, we have

$$
\begin{aligned}
\operatorname{Var}\left(b_{2, E}\right) & =4 \int_{\mathcal{D}} \int_{\mathcal{D}} r^{E}(x-y)^{2} \widetilde{r}_{1,1}^{E}(x-y)^{2} d x d y \sim \frac{9}{16} \frac{\operatorname{area}(\mathcal{D})}{\pi^{3}} \times \frac{\log E}{E}, \\
\operatorname{Cov}\left(b_{2, E}, b_{3, E}\right) & =4 \int_{\mathcal{D}} \int_{\mathcal{D}} r^{E}(x-y)^{2} \widetilde{r}_{1,2}^{E}(x-y)^{2} d x d y \sim \frac{3}{16} \frac{\operatorname{area}(\mathcal{D})}{\pi^{3}} \times \frac{\log E}{E},
\end{aligned}
$$


$\operatorname{Cov}\left(b_{2, E}, b_{4, E}\right)=4 \int_{\mathcal{D}} \int_{\mathcal{D}} \widetilde{r}_{0,1}^{E}(x-y)^{4} d x d y \sim \frac{9}{16} \frac{\operatorname{area}(\mathcal{D})}{\pi^{3}} \times \frac{\log E}{E}$,

$\operatorname{Cov}\left(b_{2, E}, b_{5, E}\right)=4 \int_{\mathcal{D}} \int_{\mathcal{D}} \widetilde{r}_{0,1}^{E}(x-y)^{2} \widetilde{r}_{0,2}^{E}(x-y)^{2} d x d y \sim \frac{3}{16} \frac{\operatorname{area}(\mathcal{D})}{\pi^{3}} \times \frac{\log E}{E}$,

$\operatorname{Cov}\left(b_{2, E}, b_{6, E}\right)=4 \int_{\mathcal{D}} \int_{\mathcal{D}} \widetilde{r}_{0,1}^{E}(x-y)^{2} \widetilde{r}_{1,1}^{E}(x-y)^{2} d x d y \sim \frac{5}{16} \frac{\operatorname{area}(\mathcal{D})}{\pi^{3}} \times \frac{\log E}{E}$,

$\operatorname{Cov}\left(b_{2, E}, b_{7, E}\right)=4 \int_{\mathcal{D}} \int_{\mathcal{D}} \widetilde{r}_{0,2}^{E}(x-y)^{2} \widetilde{r}_{1,2}^{E}(x-y)^{2} d x d y \sim \frac{1}{16} \frac{\operatorname{area}(\mathcal{D})}{\pi^{3}} \times \frac{\log E}{E}$,

$\operatorname{Cov}\left(b_{2, E}, b_{8, E}\right)=4 \int_{\mathcal{D}} \int_{\mathcal{D}} \widetilde{r}_{0,1}^{E}(x-y)^{2} \widetilde{r}_{1,2}^{E}(x-y)^{2} d x d y \sim \frac{1}{16} \frac{\operatorname{area}(\mathcal{D})}{\pi^{3}} \times \frac{\log E}{E}$.

$\operatorname{Cov}\left(b_{2, E}, b_{9, E}\right)=4 \int_{\mathcal{D}} \int_{\mathcal{D}} \widetilde{r}_{0,2}^{E}(x-y)^{2} \widetilde{r}_{1,1}^{E}(x-y)^{2} d x d y \sim \frac{1}{16} \frac{\operatorname{area}(\mathcal{D})}{\pi^{3}} \times \frac{\log E}{E}$,

$\operatorname{Cov}\left(b_{2, E}, b_{10, E}\right)=4 \int_{\mathcal{D}} \int_{\mathcal{D}} \widetilde{r}_{0,1}^{E}(x-y) \widetilde{r}_{0,2}^{E}(x-y) \tilde{r}_{1,1}^{E}(x-y) \widetilde{r}_{1,2}^{E}(x-y) d x d y$ $\sim \frac{1}{16} \frac{\operatorname{area}(\mathcal{D})}{\pi^{3}} \times \frac{\log E}{E}$.

Lemma 8.13. As $E \rightarrow+\infty$, we have

$$
\operatorname{Var}\left(b_{3, E}\right)=4 \int_{\mathcal{D}} \int_{\mathcal{D}} r^{E}(x-y)^{2} \widetilde{r}_{2,2}^{E}(x-y)^{2} d x d y \sim \frac{9}{16} \frac{\operatorname{area}(\mathcal{D})}{\pi^{3}} \times \frac{\log E}{E},
$$

$\operatorname{Cov}\left(b_{3, E}, b_{4, E}\right)=4 \int_{\mathcal{D}} \int_{\mathcal{D}} \widetilde{r}_{0,1}^{E}(x-y)^{2} \widetilde{r}_{0,2}^{E}(x-y)^{2} d x d y \sim \frac{3}{16} \frac{\operatorname{area}(\mathcal{D})}{\pi^{3}} \times \frac{\log E}{E}$,

$\operatorname{Cov}\left(b_{3, E}, b_{5, E}\right)=4 \int_{\mathcal{D}} \int_{\mathcal{D}} \widetilde{r}_{0,2}^{E}(x-y)^{4} d x d y \sim \frac{9}{16} \frac{\operatorname{area}(\mathcal{D})}{\pi^{3}} \times \frac{\log E}{E}$,

$\operatorname{Cov}\left(b_{3, E}, b_{6, E}\right)=4 \int_{\mathcal{D}} \int_{\mathcal{D}} \widetilde{r}_{0,1}^{E}(x-y)^{2} \widetilde{r}_{1,2}^{E}(x-y)^{2} d x d y \sim \frac{1}{16} \frac{\operatorname{area}(\mathcal{D})}{\pi^{3}} \times \frac{\log E}{E}$,

$\operatorname{Cov}\left(b_{3, E}, b_{7, E}\right)=4 \int_{\mathcal{D}} \int_{\mathcal{D}} \widetilde{r}_{0,2}^{E}(x-y)^{2} \widetilde{r}_{2,2}^{E}(x-y)^{2} d x d y \sim \frac{5}{16} \frac{\operatorname{area}(\mathcal{D})}{\pi^{3}} \times \frac{\log E}{E}$,

$\operatorname{Cov}\left(b_{3, E}, b_{8, E}\right)=4 \int_{\mathcal{D}} \int_{\mathcal{D}} \widetilde{r}_{0,1}^{E}(x-y)^{2} \widetilde{r}_{2,2}^{E}(x-y)^{2} d x d y \sim \frac{1}{16} \frac{\operatorname{area}(\mathcal{D})}{\pi^{3}} \times \frac{\log E}{E}$,

$\operatorname{Cov}\left(b_{3, E}, b_{9, E}\right)=4 \int_{\mathcal{D}} \int_{\mathcal{D}} \widetilde{r}_{0,2}^{E}(x-y)^{2} \widetilde{r}_{1,2}^{E}(x-y)^{2} d x d y \sim \frac{1}{16} \frac{\operatorname{area}(\mathcal{D})}{\pi^{3}} \times \frac{\log E}{E}$,

$\operatorname{Cov}\left(b_{3, E}, b_{10, E}\right)=4 \int_{\mathcal{D}} \int_{\mathcal{D}} \widetilde{r}_{0,1}^{E}(x-y) \widetilde{r}_{0,2}^{E}(x-y) \widetilde{r}_{2,2}^{E}(x-y) \widetilde{r}_{1,2}^{E}(x-y) d x d y$

$$
\sim \frac{1}{16} \frac{\operatorname{area}(\mathcal{D})}{\pi^{3}} \times \frac{\log E}{E} .
$$

Lemma 8.14. As $E \rightarrow+\infty$, we have

$$
\begin{aligned}
\operatorname{Var}\left(b_{4, E}\right) & =4 \int_{\mathcal{D}} \int_{\mathcal{D}} r^{E}(x-y)^{2} \widetilde{r}_{1,1}^{E}(x-y)^{2} d x d y \sim \frac{9}{16} \frac{\operatorname{area}(\mathcal{D})}{\pi^{3}} \times \frac{\log E}{E}, \\
\operatorname{Cov}\left(b_{4, E}, b_{5, E}\right) & \left.=4 \int_{\mathcal{D}} \int_{\mathcal{D}} r^{E}(x-y)^{2} \widetilde{r}_{1,2}^{E}(x-y)^{2}\right) d x d y \\
\operatorname{Cov}\left(b_{4, E}, b_{6, E}\right) & =4 \int_{\mathcal{D}} \int_{\mathcal{D}} \widetilde{r}_{0,1}^{E}(x-y)^{2} \widetilde{r}_{1,1}^{E}(x-y)^{2} d x d y \sim \frac{\operatorname{area}(\mathcal{D})}{\pi^{3}} \times \frac{\log E}{E},
\end{aligned}
$$


$\operatorname{Cov}\left(b_{4, E}, b_{7, E}\right)=4 \int_{\mathcal{D}} \int_{\mathcal{D}} \widetilde{r}_{0,2}^{E}(x-y)^{2} \widetilde{r}_{1,2}^{E}(x-y)^{2} d x d y \sim \frac{1}{16} \frac{\operatorname{area}(\mathcal{D})}{\pi^{3}} \times \frac{\log E}{E}$,

$\operatorname{Cov}\left(b_{4, E}, b_{8, E}\right)=4 \int_{\mathcal{D}} \int_{\mathcal{D}} \widetilde{r}_{0,2}^{E}(x-y)^{2} \widetilde{r}_{1,1}^{E}(x-y)^{2} d x d y \sim \frac{1}{16} \frac{\operatorname{area}(\mathcal{D})}{\pi^{3}} \times \frac{\log E}{E}$,

$\operatorname{Cov}\left(b_{4, E}, b_{9, E}\right)=4 \int_{\mathcal{D}} \int_{\mathcal{D}} \widetilde{r}_{0,1}^{E}(x-y)^{2} \widetilde{r}_{1,2}^{E}(x-y)^{2} d x d y \sim \frac{1}{16} \frac{\operatorname{area}(\mathcal{D})}{\pi^{3}} \times \frac{\log E}{E}$,

$\operatorname{Cov}\left(b_{4, E}, b_{10, E}\right)=4 \int_{\mathcal{D}} \int_{\mathcal{D}} \widetilde{r}_{0,1}^{E}(x-y) \widetilde{r}_{0,2}^{E}(x-y) \widetilde{r}_{1,1}^{E}(x-y) \widetilde{r}_{1,2}^{E}(x-y) d x d y$

$$
\sim \frac{1}{16} \frac{\operatorname{area}(\mathcal{D})}{\pi^{3}} \times \frac{\log E}{E} .
$$

Lemma 8.15. As $E \rightarrow+\infty$, we have

$$
\operatorname{Var}\left(b_{5, E}\right)=4 \int_{\mathcal{D}} \int_{\mathcal{D}} \widetilde{r}_{1,1}^{E}(x-y)^{4} d x d y \sim \frac{9}{16} \frac{\operatorname{area}(\mathcal{D})}{\pi^{3}} \times \frac{\log E}{E}
$$

$\operatorname{Cov}\left(b_{5, E}, b_{6, E}\right)=4 \int_{\mathcal{D}} \int_{\mathcal{D}} \widetilde{r}_{0,1}^{E}(x-y)^{2} \widetilde{r}_{1,2}^{E}(x-y)^{2} d x d y \sim \frac{1}{16} \frac{\operatorname{area}(\mathcal{D})}{\pi^{3}} \times \frac{\log E}{E}$, $\operatorname{Cov}\left(b_{5, E}, b_{7, E}\right)=4 \int_{\mathcal{D}} \int_{\mathcal{D}} \widetilde{r}_{0,2}^{E}(x-y)^{2} \widetilde{r}_{2,2}^{E}(x-y)^{2} d x d y \sim \frac{5}{16} \frac{\operatorname{area}(\mathcal{D})}{\pi^{3}} \times \frac{\log E}{E}$, $\operatorname{Cov}\left(b_{5, E}, b_{8, E}\right)=4 \int_{\mathcal{D}} \int_{\mathcal{D}} \widetilde{r}_{0,2}^{E}(x-y)^{2} \widetilde{r}_{1,2}^{E}(x-y)^{2} d x d y \sim \frac{1}{16} \frac{\operatorname{area}(\mathcal{D})}{\pi^{3}} \times \frac{\log E}{E}$, $\operatorname{Cov}\left(b_{5, E}, b_{9, E}\right)=4 \int_{\mathcal{D}} \int_{\mathcal{D}} \widetilde{r}_{0,1}^{E}(x-y)^{2} \widetilde{r}_{2,2}^{E}(x-y)^{2} d x d y \sim \frac{1}{16} \frac{\operatorname{area}(\mathcal{D})}{\pi^{3}} \times \frac{\log E}{E}$, $\operatorname{Cov}\left(b_{5, E}, b_{10, E}\right)=4 \int_{\mathcal{D}} \int_{\mathcal{D}} \widetilde{r}_{0,1}^{E}(x-y) \widetilde{r}_{0,2}^{E}(x-y) \widetilde{r}_{2,2}^{E}(x-y) \widetilde{r}_{1,2}^{E}(x-y) d x d y$ $\sim \frac{1}{16} \frac{\operatorname{area}(\mathcal{D})}{\pi^{3}} \times \frac{\log E}{E}$.

Lemma 8.16. As $E \rightarrow+\infty$, we have

$$
\begin{aligned}
\operatorname{Var}\left(b_{6, E}\right) & =4 \int_{\mathcal{D}} \int_{\mathcal{D}} \widetilde{r}_{1,1}^{E}(x-y)^{4} d x d y \sim \frac{105}{64} \frac{\operatorname{area}(\mathcal{D})}{\pi^{3}} \times \frac{\log E}{E}, \\
\operatorname{Cov}\left(b_{6, E}, b_{7, E}\right) & =4 \int_{\mathcal{D}} \int_{\mathcal{D}} \widetilde{r}_{1,2}^{E}(x-y)^{4} d x d y \sim \frac{9}{64} \frac{\operatorname{area}(\mathcal{D})}{\pi^{3}} \times \frac{\log E}{E}, \\
\operatorname{Cov}\left(b_{6, E}, b_{8, E}\right) & =4 \int_{\mathcal{D}} \int_{\mathcal{D}} \widetilde{r}_{1,1}^{E}(x-y)^{2} \widetilde{r}_{1,2}^{E}(x-y)^{2} d x d y \sim \frac{15}{64} \frac{\operatorname{area}(\mathcal{D})}{\pi^{3}} \times \frac{\log E}{E}, \\
\operatorname{Cov}\left(b_{6, E}, b_{9, E}\right) & \left.=4 \int_{\mathcal{D}} \int_{\mathcal{D}} \widetilde{r}_{1,1}^{E}(x-y)^{2} \widetilde{r}_{1,2}^{E}(x-y)^{2}\right) d x d y \sim \frac{15}{64} \frac{\operatorname{area}(\mathcal{D})}{\pi^{3}} \times \frac{\log E}{E} \\
\operatorname{Cov}\left(b_{6, E}, b_{10, E}\right) & =4 \int_{\mathcal{D}} \int_{\mathcal{D}} \widetilde{r}_{1,1}^{E}(x-y)^{2} \widetilde{r}_{1,2}^{E}(x-y)^{2} d x d y \sim \frac{15}{64} \frac{\operatorname{area}(\mathcal{D})}{\pi^{3}} \times \frac{\log E}{E} .
\end{aligned}
$$


Lemma 8.17. As $E \rightarrow+\infty$, we have

$$
\begin{aligned}
\operatorname{Var}\left(b_{7, E}\right)=4 & \int_{\mathcal{D}} \int_{\mathcal{D}} \widetilde{r}_{2,2}^{E}(x-y)^{4} d x d y \\
& \sim \frac{105}{64} \frac{\operatorname{area}(\mathcal{D})}{\pi^{3}} \times \frac{\log E}{E}, \\
\operatorname{Cov}\left(b_{7, E}, b_{8, E}\right)=4 & \int_{\mathcal{D}} \int_{\mathcal{D}} \widetilde{r}_{2,2}^{E}(x-y)^{2} \widetilde{r}_{1,2}^{E}(x-y)^{2} d x d y \\
& \sim \frac{15}{64} \frac{\operatorname{area}(\mathcal{D})}{\pi^{3}} \times \frac{\log E}{E}, \\
\operatorname{Cov}\left(b_{7, E}, b_{9, E}\right)=4 & \int_{\mathcal{D}} \int_{\mathcal{D}} \widetilde{r}_{2,2}^{E}(x-y)^{2} \widetilde{r}_{1,2}^{E}(x-y)^{2} d x d y \\
& \sim \frac{15}{64} \frac{\operatorname{area}(\mathcal{D})}{\pi^{3}} \times \frac{\log E}{E}, \\
\operatorname{Cov}\left(b_{7, E}, b_{10, E}\right)=4 & \int_{\mathcal{D}} \int_{\mathcal{D}} \widetilde{r}_{2,2}^{E}(x-y)^{2} \widetilde{r}_{1,2}^{E}(x-y)^{2} d x d y \\
& \sim \frac{15}{64} \frac{\operatorname{area}(\mathcal{D})}{\pi^{3}} \times \frac{\log E}{E} .
\end{aligned}
$$

Lemma 8.18. As $E \rightarrow+\infty$, we have

$$
\begin{aligned}
\operatorname{Var}\left(b_{8, E}\right)= & \int_{\mathcal{D}} \int_{\mathcal{D}} \widetilde{r}_{1,1}^{E}(x-y)^{2} \widetilde{r}_{2,2}^{E}(x-y)^{2} d x d y \\
& \sim \frac{9}{64} \frac{\operatorname{area}(\mathcal{D})}{\pi^{3}} \times \frac{\log E}{E} \\
\operatorname{Cov}\left(b_{8, E}, b_{9, E}\right)=4 & \int_{\mathcal{D}} \int_{\mathcal{D}} \widetilde{r}_{1,2}^{E}(x-y)^{4} d x d y \\
& \sim \frac{9}{64} \frac{\operatorname{area}(\mathcal{D})}{\pi^{3}} \times \frac{\log E}{E}, \\
\operatorname{Cov}\left(b_{8, E}, b_{10, E}\right)=4 & \int_{\mathcal{D}} \int_{\mathcal{D}} \widetilde{r}_{1,1}^{E}(x-y) \widetilde{r}_{2,2}^{E}(x-y) \widetilde{r}_{1,2}^{E}(x-y)^{2} d x d y \\
& \sim \frac{9}{64} \frac{\operatorname{area}(\mathcal{D})}{\pi^{3}} \times \frac{\log E}{E} .
\end{aligned}
$$

Lemma 8.19. As $E \rightarrow+\infty$, we have

$$
\begin{aligned}
\operatorname{Var}\left(b_{9, E}\right)=4 & \int_{\mathcal{D}} \int_{\mathcal{D}} \widetilde{r}_{1,1}^{E}(x-y)^{2} \widetilde{r}_{2,2}^{E}(x-y)^{2} d x d y \\
& \sim \frac{9}{64} \frac{\operatorname{area}(\mathcal{D})}{\pi^{3}} \times \frac{\log E}{E}, \\
\operatorname{Cov}\left(b_{9, E}, b_{10, E}\right)=4 & \int_{\mathcal{D}} \int_{\mathcal{D}} \widetilde{r}_{1,1}^{E}(x-y) \widetilde{r}_{2,2}^{E}(x-y) \widetilde{r}_{1,2}^{E}(x-y)^{2} d x d y \\
& \sim \frac{9}{64} \frac{\operatorname{area}(\mathcal{D})}{\pi^{3}} \times \frac{\log E}{E},
\end{aligned}
$$




$$
\begin{aligned}
\operatorname{Var}\left(b_{9, E}\right)= & \int_{\mathcal{D}} \int_{\mathcal{D}}\left(\widetilde{r}_{1,1}^{E}(x-y)^{2} \widetilde{r}_{2,2}^{E}(x-y)^{2}\right. \\
& +2 \widetilde{r}_{1,1}^{E}(x-y) \widetilde{r}_{2,2}^{E}(x-y) \widetilde{r}_{1,2}^{E}(x-y)^{2} \\
& \left.+\widetilde{r}_{1,2}^{E}(x-y)^{4}\right) d x d y \sim \frac{9}{64} \frac{\operatorname{area}(\mathcal{D})}{\pi^{3}} \times \frac{\log E}{E} .
\end{aligned}
$$

\section{Appendix C}

Proof of Lemma 7.6. Reasoning as in the proof of Proposition 5.1, we have

$$
\begin{aligned}
\int_{\mathcal{D}} \int_{\mathcal{D}} \widetilde{r}_{k, l}^{E}(x-y)^{6} d x d y= & \operatorname{area}(\mathcal{D}) \int_{0}^{\operatorname{diam}(\mathcal{D})} d \phi \phi \int_{0}^{2 \pi} \widetilde{r}_{k, l}^{E}(\phi \cos \theta, \phi \sin \theta)^{6} d \theta \\
& +O\left(\int_{0}^{\operatorname{diam}(\mathcal{D})} d \phi \phi^{2} \int_{0}^{2 \pi} \widetilde{r}_{k, l}^{E}(\phi \cos \theta, \phi \sin \theta)^{6} d \theta\right) .
\end{aligned}
$$

Performing the change of variable $\theta=\psi / \sqrt{E}$ in the first term on the r.h.s. of (8.118) we obtain

$$
\begin{aligned}
& \operatorname{area}(\mathcal{D}) \int_{0}^{\operatorname{diam}(\mathcal{D})} d \phi \phi \int_{0}^{2 \pi} \tilde{r}_{k, l}^{E}(\phi \cos \theta, \phi \sin \theta)^{6} d \theta \\
& \quad=\operatorname{area}(\mathcal{D}) \frac{1}{E} \int_{0}^{\sqrt{E} \cdot \operatorname{diam}(\mathcal{D})} d \psi \psi \int_{0}^{2 \pi} \widetilde{r}_{k, l}^{1}(\psi \cos \theta, \psi \sin \theta)^{6} d \theta .
\end{aligned}
$$

Since $r^{1}(\psi \cos \theta, \psi \sin \theta) \rightarrow 1, \widetilde{r}_{0, i}^{1}(\psi \cos \theta, \psi \sin \theta)=O(\psi)$ and $\widetilde{r}_{i, i}^{1}(\psi \cos \theta, \psi \sin \theta)$ $\rightarrow 1, \widetilde{r}_{1,2}^{1}(\psi \cos \theta, \psi \sin \theta)=O\left(\psi^{2}\right)$ as $\psi \rightarrow 0$ uniformly on $\theta(i=1,2)$, then we can rewrite (8.119) as

$$
\begin{aligned}
& \operatorname{area}(\mathcal{D}) \frac{1}{E} \int_{0}^{\sqrt{E} \cdot \operatorname{diam}(\mathcal{D})} d \psi \psi \int_{0}^{2 \pi} \widetilde{r}_{k, l}^{1}(\psi \cos \theta, \psi \sin \theta)^{6} d \theta \\
& \quad=O\left(\frac{1}{E}\right)+\operatorname{area}(\mathcal{D}) \frac{1}{E} \int_{1}^{\sqrt{E} \cdot \operatorname{diam}(\mathcal{D})} d \psi \psi \int_{0}^{2 \pi} \widetilde{r}_{k, l}^{1}(\psi \cos \theta, \psi \sin \theta)^{6} d \theta
\end{aligned}
$$

Now using (5.63) for the second term on the r.h.s. of (8.120), as $E \rightarrow+\infty$, we have

$$
\begin{aligned}
& \frac{1}{E} \int_{1}^{\sqrt{E} \cdot \operatorname{diam}(\mathcal{D})} d \psi \psi \int_{0}^{2 \pi} \tilde{r}_{k, l}^{1}(\psi \cos \theta, \psi \sin \theta)^{6} d \theta \\
& \ll \frac{1}{E} \int_{1}^{\sqrt{E} \cdot \operatorname{diam}(\mathcal{D})} \frac{d \psi}{\psi^{2}} \sim \frac{1}{E} .
\end{aligned}
$$

For the error term on the r.h.s. of (8.118) an analogous argument yields, as $E \rightarrow+\infty$,

$$
\int_{0}^{\operatorname{diam}(\mathcal{D})} d \phi \phi^{2} \int_{0}^{2 \pi} \widetilde{r}_{k, l}^{E}(\phi \cos \theta, \phi \sin \theta)^{6} d \theta \asymp \frac{\log E}{E \sqrt{E}} .
$$

Thanks to (8.121) and (8.122), (8.118) concludes the proof. 
From (8.108), the covariance matrix of $\nabla B_{E}(x)$ conditioned to $B_{E}(x)=B_{E}(0)=0$ is

$$
\Omega_{E}(x)=2 \pi^{2} E I_{2}-\frac{\nabla r^{E}(x)^{t} \nabla r^{E}(x)}{1-r^{E}(x)^{2}},
$$

and its determinant is

$$
\operatorname{det}\left(\Omega^{E}(x)\right)=2 \pi^{2} E\left(2 \pi^{2} E-\frac{\left\|\nabla r^{E}(x)\right\|^{2}}{1-r^{E}(x)^{2}}\right) .
$$

Lemma 8.20. As $x \rightarrow 0$, it holds

$$
\Psi_{E}(x):=\frac{\left|\operatorname{det}\left(\Omega_{E}(x)\right)\right|}{1-r^{E}(x)^{2}}=\frac{1}{8}\left(2 \pi^{2} E\right)^{2}+E^{3} O\left(\|x\|^{2}\right),
$$

where the constant involved in the "O"-notation does not depend on $E$.

Proof. The Taylor development of $r^{E}$ centered at 0 is

$$
r^{E}(x)=1-2 \pi^{2} E \frac{\|x\|^{2}}{2}+\frac{\left(2 \pi^{2} E\right)^{2}\|x\|^{4}}{16}+E^{3} O\left(\|x\|^{6}\right),
$$

where, from now until the end of the proof, the constants involved in the "O"-notation do not depend on $E$. From (8.123) it is immediate that

$$
1-r^{E}(x)^{2}=2 \pi^{2} E\|x\|^{2}-\frac{3}{8}\left(2 \pi^{2} E\right)^{2}\|x\|^{4}+E^{3} O\left(\|x\|^{6}\right) .
$$

Analogously, we find that the Taylor development for $\left\|\nabla r^{E}(x)\right\|^{2}$ centered at 0 is

$$
\left\|\nabla r^{E}(x)\right\|^{2}=2 \pi^{2} E\left(2 \pi^{2} E\|x\|^{2}+\left(2 \pi^{2} E\right)^{2} \frac{\|x\|^{4}}{2}+E^{3} O\left(\|x\|^{6}\right)\right) .
$$

From (8.124) and (8.125) we get

$$
\begin{aligned}
\frac{\left\|\nabla r^{E}(x)\right\|^{2}}{1-r^{E}(x)^{2}}= & \frac{2 \pi^{2} E\left(2 \pi^{2} E\|x\|^{2}+\left(2 \pi^{2} E\right)^{2} \frac{\|x\|^{4}}{2}+E^{3} O\left(\|x\|^{6}\right)\right)}{2 \pi^{2} E\|x\|^{2}-\frac{3}{8}\left(2 \pi^{2} E\right)^{2}\|x\|^{4}+E^{3} O\left(\|x\|^{6}\right)} \\
= & \frac{\left(2 \pi^{2} E\right)^{2}\|x\|^{2}\left(1+2 \pi^{2} E \frac{\|x\|^{2}}{2}+E^{2} O\left(\|x\|^{4}\right)\right)}{2 \pi^{2} E\|x\|^{2}\left(1-2 \pi^{2} E \frac{3}{8}\|x\|^{2}+E^{2} O\left(\|x\|^{4}\right)\right)} \\
= & 2 \pi^{2} E\left(1+2 \pi^{2} E \frac{\|x\|^{2}}{2}+E^{2} O\left(\|x\|^{4}\right)\right) \\
& \times\left(1-2 \pi^{2} E \frac{3}{8}\|x\|^{2}+E^{2} O\left(\|x\|^{4}\right)\right) \\
= & 2 \pi^{2} E\left(1-2 \pi^{2} E \frac{1}{8}\|x\|^{2}+E^{2} O\left(\|x\|^{4}\right)\right) .
\end{aligned}
$$


From (8.126) and using again (8.124) we can write

$$
\begin{aligned}
\Psi_{E}(x) & =\frac{\left|2 \pi^{2} E\left(2 \pi^{2} E-\frac{\left\|\nabla r^{E}(x)\right\|^{2}}{1-r^{E}(x)^{2}}\right)\right|}{1-k_{E}(x)^{2}} \\
& =\frac{\left(2 \pi^{2} E\right)^{3} \frac{1}{8}\|x\|^{2}+E^{4} O\left(\|x\|^{4}\right)}{2 \pi^{2} E\|x\|^{2}+E^{2} O\left(\|x\|^{4}\right)} \\
& =\frac{1}{8}\left(2 \pi^{2} E\right)^{2}\left(1+E O\left(\|x\|^{2}\right)\right)
\end{aligned}
$$

which conclude the proof.

\section{References}

[AS64] Abramowitz, M., Stegun, I.A.: Handbook of Mathematical Functions with Formulas, Graphs, and Mathematical Tables. National Bureau of Standards Applied Mathematics Series, Washington, DC (1964)

[AT] Adler, R.J., Taylor, J.E.: Random Fields and Geometry. Springer Monographs in Mathematics, New York (2007)

[APP16] Angst, J., Poly, G., Pham, V.H.: Universality of the nodal length of bivariate random trigonometric polynomials. Trans. Am. Math. Soc. 370(12), 8331-8357 (2018)

[AW] Azaïs, J.-M., Wschebor, M.: Level Sets and Extrema of Random Processes and Fields. Wiley, Hoboken (2009)

[Bera85] Bérard, P.: Volume des ensembles nodaux des fonctions propres du laplacien. Bony-SjostrandMeyer seminar, Exp. No. 14, 10 pp., École Polytechnique, Palaiseau (1985)

[Ber77] Berry, M.V.: Regular and irregular semiclassical wavefunctions. J. Phys. A 10(12), 2083-2091 (1977)

[Ber02] Berry, M.V.: Statistics of nodal lines and points in chaotic quantum billiards: perimeter corrections, fluctuations, curvature. J. Phys. A 35(13), 3025-3038 (2002)

[Cam17] Cammarota, V.: Nodal area distribution for arithmetic random waves. Transactions of the American Mathematical Society, in press (preprint arXiv:1708.07679) (2019+)

[CMW16a] Cammarota, V., Marinucci, D., Wigman, I.: On the distribution of the critical values of random spherical harmonics. J. Geom. Anal. 4, 3252-3324 (2016)

[CMW16b] Cammarota, V., Marinucci, D., Wigman, I.: Fluctuations of the Euler-Poincaré characteristic for random spherical harmonics. Proc. Am. Math. Soc. 144(11), 4759-4775 (2016)

[CM16] Cammarota, V., Marinucci, D.: A quantitative central limit theorem for the Euler-Poincaré characteristic of random spherical eigenfunctions. Ann. Probab. 46(6), 3188-3228 (2018)

[CH16a] Canzani, Y., Hanin, B.: Local universality for zeros and critical points of monochromatic random waves, Preprint arXiv:1610.09438 (2016)

[CH16b] Canzani, Y., Hanin, B.: $C^{\infty}$ scaling asymptotics for the spectral projector of the Laplacian. J. Geom. Anal. 28(1), 111-122 (2018)

[Che76] Cheng, S.Y.: Eigenfunctions and nodal sets. Comment. Math. Helv. 5, 43-55 (1976)

[DNPR16] Dalmao, F., Nourdin, I., Peccati, G., Rossi, M.: Phase singularities in complex arithmetic random waves, Preprint arXiv:1608.05631 (2016)

[DOP09] Dennis, M.R., O’Holleran, K., Padgett, M.J.: Singular optics: optical vortices and polarization singularities. In: Progress in Optics, Vol. 53., Elsevier, Amsterdam, pp. 293-363 (2009)

[DF88] Donnelly, H., Fefferman, C.: Nodal sets of eigenfunctions on Riemannian manifolds. Invent. Math. 93, 161-183 (1988)

[Kra14] Krasikov, I.: Approximations for the Bessel and Airy functions with an explicit error term. LMS J. Comput. Math. 17(1), 209-22 (2014)

[KKW13] Krishnapur, M., Kurlberg, P., Wigman, I.: Nodal length fluctuations for arithmetic random waves. Ann. Math. (2) 177(2), 699-737 (2013)

[KW16] Kurlberg, P., Wigman, I.: On probability measures arising from lattice points on circles. Math. Ann. 367(3-4), 1057-1098 (2017)

[Lee97] Lee, J.M.: Riemannian Manifolds: An Introduction to Curvature, Graduate Texts in Mathematics, 176. Springer, New York (1997)

[MP11] Marinucci, D., Peccati, G.: Random Fields on the Sphere: Representations, Limit Theorems and Cosmological Applications. Cambridge University Press, Cambridge (2011) 
[MW11] Marinucci, D., Wigman, I.: On the area of excursion sets of spherical Gaussian eigenfunctions. J. Math. Phys. 52(9), 093301 (2011)

[MPRW16] Marinucci, D., Peccati, G., Rossi, M., Wigman, I.: Non-universality of nodal length distribution for arithmetic random waves. Geom. Funct. Anal. 3, 926-960 (2016)

[MRW17] Marinucci, D., Rossi, M., Wigman, I.: The asymptotic equivalence of the sample trispectrum and the nodal length for random spherical harmonics. Annales de l'Institut Henri Poincaré, Probability and Statistics (2017). in press

[NP12] Nourdin, I, Peccati, G.: Normal approximations with Malliavin calculus: from Stein's method to universality. Cambridge Tracts in Mathematics, 192. Cambridge University Press, Cambridge (2012)

[NuPe05] Nualart, D., Peccati, G.: Central limit theorems for sequences of multiple stochastic integrals. Ann. Probab. 33(1), 177-193 (2005)

[ORW08] Oravecz, F., Rudnick, Z., Wigman, I.: The Leray measure of nodal sets for random eigenfunctions on the torus. Ann. Inst. Fourier (Grenoble) 58(1), 299-335 (2008)

[PR17] Peccati, G, Rossi, M.: Quantitative limit theorems for local functionals of arithmetic random waves. In: Computation and Combinatorics in Dynamics, Stochastics and Control, The Abel Symposium, Rosendal, Norway, August 2016, 13, 659-689 (2018)

[PT05] Peccati, G., Tudor, C.A.: Gaussian limits for vector-valued multiple stochastic integrals. In: Séminaire de Probabilités XXXVIII, 247-262, Lecture Notes in Math., 1857, Springer, Berlin (2005)

[Ros15] Rossi, M.: The geometry of spherical random fields, Ph.D. thesis, University of Rome "Tor Vergata", arXiv:1603.07575 (2015)

[RoW17] Rossi, M., Wigman, I.: Asymptotic distribution of nodal intersections for arithmetic random waves. Nonlinearity 31, 4472 (2018)

[RW08] Rudnick, Z., Wigman, I.: On the volume of nodal sets for eigenfunctions of the Laplacian on the torus. Ann. Henri Poincaré 1(9), 109-130 (2008)

[RW16] Rudnick, Z., Wigman, I.: Nodal intersections for random eigenfunctions on the torus. Am. J. Math. 138(6), 1605-1644 (2016)

[Sze75] Szego, G.: Orthogonal polynomials. Fourth edition. American Mathematical Society, Colloquium Publications, Vol. XXIII. American Mathematical Society, Providence, RI (1975)

[UR13] Urbina, J., Richter, K.: Random quantum states: recent developments and applications. Adv. Phys. 62, 363-452 (2013)

[Wig10] Wigman, I.: Fluctuations of the nodal length of random spherical harmonics. Commun. Math. Phys. 298(3), 787-831 (2010)

[Zel09] Zelditch, S.: Real and complex zeros of Riemannian random waves. Contemp. Math. 14, 321 (2009)

Communicated by M. Hairer 